Submitted to ApJSuppl

\title{
THE FORMATION RATE, MASS AND LUMINOSITY FUNCTIONS OF DA WHITE DWARFS FROM THE PALOMAR GREEN SURVEY
}

\author{
James Liebert \\ Steward Observatory, University of Arizona, Tucson AZ 85726 \\ liebert@as.arizona.edu \\ P. Bergeron \\ Département de Physique, Université de Montréal, \\ C.P. 6128, Succ. Centre-Ville, Montréal, Québec, \\ Canada $\mathrm{H} 3 \mathrm{C} 3 \mathrm{~J}^{\eta}$ \\ bergeron@astro umontreal.ca \\ and \\ J.B. Holberg \\ Lunar and Planetary Laboratory, University of Arizona \\ Tucson, AZ 85726 \\ holberg@argus.lpl.arizona.edu
}

\begin{abstract}
Spectrophotometric observations at high signal-to-noise ratio were obtained of a complete sample of 347 DA white dwarfs from the Palomar Green (PG) Survey. Fits of observed Balmer lines to synthetic spectra calculated from pure-hydrogen model atmospheres were used to obtain robust values of $T_{\text {eff }}, \log g$, masses, radii, and cooling ages. The luminosity function of the sample, weighted by $1 / V_{\max }$, was obtained and compared with other determinations. Incompleteness of the sample due to selection by photographic $m_{u}-m_{b}$ color and magnitude limits was
\end{abstract}


found to be a serious problem, and an attempt is made to correct for this. The mass distribution of the white dwarfs is derived, after important corrections for the radii of the white dwarfs in this magnitude-limited survey and for the cooling time scales. This distribution has (1) a "peak" component centered near $0.6 M_{\odot}$, (2) a low mass component centered near $0.4 M_{\odot}$, and (3) a high mass component above about $0.8 M_{\odot}$. The formation rate of DA white dwarfs from the PG is estimated to be $0.6 \times 10^{-12} \mathrm{pc}^{-3} \mathrm{yr}^{-1}$. Of these, $75 \%$ are from the peak component, $10 \%$ from the low mass component, and $15 \%$ from the high mass component. The low mass component requires binary evolution for $100 \%$ of the objects, with a degenerate companion likely in the majority of cases. Comparison with predictions from a theoretical study of the white dwarf formation rate for single stars indicates that $\geq 80 \%$ of the high mass component requires a different origin, presumably mergers of lower mass double degenerate stars. The need for a binary channel may not be as great for the massive, very hot white dwarfs found in the EUV all-sky surveys. In an Appendix, we even suggest that an enhanced density of the massive white dwarfs at lower Galactic latitudes might be due to some of them being the progeny of B stars in Gould's Belt. In order to estimate the recent formation rate of all white dwarfs in the local Galactic disk, corrections for incompleteness of the PG, addition of the DB-DO white dwarfs, and allowance for stars hidden by luminous binary companions had to be applied to enhance the rate. An overall formation rate of white dwarfs recently in the local Galactic disk of $1 \pm 0.25 \times 10^{-12} \mathrm{pc}^{-3} \mathrm{yr}^{-1}$ is obtained. Admittedly, the systematic errors in this estimate are difficult to quantify. Two recent studies of samples of nearby Galactic planetary nebulae lead to estimates around twice as high. Difficulties in reconciling these determinations are discussed.

Subject headings: white dwarfs - stars: fundamental properties - stars: luminosity function, mass function - solar neighborhood

\section{INTRODUCTION}

The luminosity and mass functions ( $\mathrm{LF} / \mathrm{MF}$ ) derived from a complete sample of hot white dwarfs in the solar neighborhood contain a variety of useful information on the star formation history of the local Galactic disk and on stellar evolution. First, the LF is a direct measure of the current death rate of stars in the local disk. Secondly, the mass distribution of a volume-defined and complete sample reveals the amount of mass lost during stellar evolution from an initial stellar mass distribution. Moreover, the MF can also reveal the 
roles of close binary evolution in forming some of the white dwarfs.

The Palomar Green (PG) Survey (Green et al. 1986) provides a complete, magnitude and photographic $m_{u}-m_{b}$ color limited survey covering nearly one quarter of the sky at Galactic latitudes $b \geq 30$ degrees. The estimation of the DA white dwarf formation rate and luminosity function from the entire area of this survey (Fleming et al. 1986) utilized photographic, image tube and vidicon spectra, Strömgren and multichannel spectrophotometry, and correspondingly limited analysis techniques - i.e. the assumption of a mean mass of $0.6 M_{\odot}$, and the estimates of $T_{\text {eff }}$ using a variety of colors and $\mathrm{H} \beta$ equivalent width measurements. Moreover, a significant fraction of stars in the PG Survey classified originally as DA white dwarfs turn out to be lower surface gravity stars (and a few migrated the other direction).

In this reanalysis, all valid DA stars from the survey were reobserved with optical CCD spectra. Hydrogen Balmer line profiles through $\mathrm{H} \beta$ were fit with model atmosphere models to determine the effective temperature and surface gravity of the star and estimate the mass following the procedures of Bergeron et al. (1992, hereafter BSL). The evolutionary models of Wood $(1990,1995)$ were used to provide a second relation between radius and mass at the derived $T_{\text {eff }}$, so that individual stellar parameters for each star could be obtained. For several stars in this sample not reobserved, we will utilize similar fits by other research groups (see below), with which we will otherwise compare our results.

Various other, large samples of hot DA stars have recently been analyzed using primarily the Balmer line fitting technique to obtain the stellar and atmospheric parameters. Finley et al. (1997) analyzed 174 stars selected from catalogs of known white dwarfs and those newly-discovered in space-based extreme ultraviolet radiation surveys. Similarly, Marsh et al. (1997) performed a similar analysis of the optical spectrophotometry of the sample of 89 hot DA stars discovered in the ROSAT all-sky survey, although many of these detections were previously known stars. Likewise, Vennes et al. (1997b) analyzed 110 stars discovered or rediscovered in the similar all-sky survey of the Extreme Ultraviolet Explorer (EUVE), with special emphasis on analyzing the mass distribution, and making comparison with stars selected optically (such as this study). We shall compare our parameter determinations with those from the above studies, in order to determine if there are systematic differences in the $T_{\text {eff }}$ and $\log g$ values, along with the resulting derived parameters.

The EUV-derived studies have demonstrated the wide variation in the EUV opacity at a given $T_{\text {eff }}$ among those with nearly-pure hydrogen atmospheres, vs. those with strong traces of heavier elements. This of course has called into question the ability to use an EUVselected sample to define a volume-limited, complete sample of white dwarfs - cf. Finley et al. (1997); Marsh et al. (1997). However, we will bring possible new insight on this question 
in this paper.

In this paper, we shall review in $\S 2$ the input data set, model atmosphere assumptions and physics, and fitting technique. In $\S 2.4$ the derived $T_{\text {eff }}, \log g$, mass and radius values are presented, as are the estimated cooling ages from the models of Wood (1995) and Althaus et al. (2001). Comparison of the overlapping samples with the results of other studies is made in $\S 2.5$. The $\mathrm{LF}$ and $\mathrm{MF}$ are presented in $\S 3$. It is useful to discuss the LF and other distribution functions in terms of three mass components - a low mass component centered near $0.4 M_{\odot}$, the dominant $0.6 M_{\odot}$ "peak" component, and a high mass component at $>0.8 M_{\odot}$. The completeness of the PG sample, a difficult issue, is discussed in $\S 4$. Then in $\S 5$ we arrive at the most fundamental results of this analysis, the formation rates of PG DA white dwarf stars for recent times in the local Galactic disk. These rates are determined for the three mass components defined in $\S 3$. To estimate the total, recent formation rate of white dwarf stars - or the "death rate" of stars of low and intermediate masses - it is necessary to increment the results for DB/DO stars ( $\S 5.2)$, for survey incompleteness ( $\S 5.3)$, and for white dwarfs hidden by luminous companions ( $\S 5.4)$. The total white dwarf formation rate is given in $(\S 5.5)$. We then compare in $\S 5.6$ our formation rate estimate with other determinations for white dwarfs and for planetary nebulae. In $\S 6$ we attempt a quantitative assessment of the fractions of the component of massive white dwarfs formed from (1) single progenitors of high initial mass, and (2) presumably, a binary white dwarf channel. In $\S 7$ we comment on objects of special spectral characteristics - magnetic white dwarfs, DAB stars in and near 30-45,000 K (the so-called "DB gap") and pulsating ZZ Ceti stars. Finally, in $\S 8$ is a brief summary of this study, and its likely value as a "benchmark" for future studies of much larger samples of hot white dwarfs.

\section{OBSERVATIONS, MODELS, FITTING TECHNIQUE, AND DERIVED PARAMETERS}

\subsection{Sample Selection}

Our goal was to observe with current $\mathrm{CCD}$ detectors at high $\mathrm{S} / \mathrm{N}$ ratio, a complete sample of DA stars from the PG Survey. The PG catalog contains a total of 420 DA stars, 363 of which are part of the complete sample ${ }^{1}$. Upon reobservation, 27 of these were in fact misclassifications, generally turning out to be subdwarf B or OB stars including at least

\footnotetext{
${ }^{1}$ For the PG DA stars discussed in this paper, finding charts, accurate coordinates, and summaries of prior spectroscopic analyses are available on the White Dwarf Database at http://procyon.lpl.arizona.edu/WD/
} 
one with a G-K companion (PG 0009+191, 0026+136, 0111+177, 0221+217, 0910+622, $0947+639,1025+258,1035+532,1121+145,1134+124,1236+479,1247+554,1302+284$, $1308-099,1347+254,1430+427,1450+432,1525+422,1538+269,1539+530,1630+249$, 2204+071, 2246+154, and 2333-002), subdwarf O stars (PG 0035+125 and 1138+424), and a DC white dwarf (PG 1055-073). One of these, PG 2204+071, has two entries in the PG catalog with almost identical coordinates, classified DA and DA5; only the southern and fainter object is a genuine DA star while the other star corresponds to a sdOB star. Major studies of sdB (Saffer et al. 1994) and sdO stars (Thejll et al. 1994), and the identification of candidate B main sequence stars at high galactic latitude (Saffer et al. 1997) led to the reobservation of most PG stars originally classified as lower gravity objects. Twelve of these proved to be DA white dwarfs (and one a DO white dwarf). Thus, while we cannot guarantee that a few DA stars do not continue to lurk among the samples originally classified $\mathrm{sdB}, \mathrm{sdO}$ or otherwise, we are convinced that the number is a few at most. A more important issue, to be addressed later, is whether the PG catalog is itself significantly incomplete.

We ended up with a complete sample of 347 genuine DA stars to be analyzed using the spectroscopic technique. Optical spectra for this sample have been gathered over the last 10 years from various sources. About $20 \%$ of them have been kindly provided to us by C. Moran (private communication), while the rest has been secured using the $2.3 \mathrm{~m}$ telescope at the Steward Observatory Kitt Peak Station, equipped with the Boller \& Chivens spectrograph and a Texas Instrument CCD detector with $15 \mu$ pixels. The spectral coverage is about $\lambda \lambda 3100-5300$, thus covering $\mathrm{H} \beta$ up to $\mathrm{H} 9$ at an intermediate resolution of $\sim 6 \AA \mathrm{FWHM}$. The spectra from $\mathrm{C}$. Moran have a similar spectral coverage but a higher resolution of $3 \AA$ FWHM. Over 100 stars have been observed twice or more in order to estimate the external error of the atmospheric parameter determinations (see below). No spectroscopic observations were obtained for the strongly magnetic white dwarfs PG 1031+234 (Schmidt et al. 1986) and PG 1533-057 (Liebert et al. 1985) since these cannot be analyzed within our theoretical framework, and the atmospheric parameters for these stars will thus be taken from the literature.

The distribution of signal-to-noise (S/N) ratios for our 345 optical spectra is displayed in Figure 1. The $\mathrm{S} / \mathrm{N}$ is based on estimates of the RMS noise per pixel in the continuum. About $50 \%$ of our sample has $\mathrm{S} / \mathrm{N}>80$ and $90 \%$ above 50 . Only the faintest objects in the sample have a lower signal-to-noise ratio. Sample spectra covering the entire temperature range of the $\mathrm{PG}$ sample are displayed in Figure 2. Effective temperatures range from above 100,000 K down to $~ 7000 \mathrm{~K}$. Examples of DAO (PG 1252+378), magnetic (PG 2329+267), and composite (PG 1314+294) white dwarfs are shown. Note the strong contrast between the high gravity white dwarf PG 1058-129 and the adjacent normal gravity white dwarfs of comparable effective temperatures, PG $1553+354$ and PG 1713+333. 


\section{2. $\quad$ Model Spectra}

The sensitivity of Balmer line profiles to $T_{\text {eff }}$ and $\log g$ has been known for a long time (c.f. Wegner \& Schulz 1981). This was first exploited using new generations of both CCD spectrophotometry and models incorporating the Hummer \& Mihalas (1988) occupation probability formalism for hydrogen by Bergeron et al. (1990, 1991). Refinements to the Hummer \& Mihalas formalism were utilized in the first major study of warm/hot DA white dwarfs by BSL. The BSL study of the mass distribution of white dwarfs was generally restricted to the $T_{\text {eff }}$ range $15,000-40,000 \mathrm{~K}$ because of problems with the accuracy of the models both above and below this range.

For the cooler stars, the problem is posed by the onset of significant atmospheric convection. The derived $T_{\text {eff }}$ and $\log g$ values are sensitive to the input parameters of the mixing length theory, though Bergeron et al. (1995) find that the ML2/ $\alpha=0.6$ formulation appears to yield the best internal consistency between optical and UV effective temperatures, trigonometric parallaxes, $V$ magnitudes, and gravitational redshifts. This formulation is therefore adopted for this study, which includes stars as low in temperature as approximately $7000 \mathrm{~K}$.

For stars hotter than about 40,000 K several complications must be acknowledged that compromise the accuracy of these results, which generally assume pure hydrogen atmospheres unless helium is visible in the spectrum (a DAO, or DAB classification). First, even if helium is not detected in the form of a He II $4686 \AA$ line, it may be present in an abundance sufficient to affect significantly the atmospheric structure and Balmer line profiles of a very hot DA star according to the LTE analysis of Bergeron et al. (1994). However, Napiwotzki (1997) has shown that this conclusion is an artifact caused by the assumption of LTE, and the influence of small traces of helium on the Balmer profiles vanishes when NLTE effects are taken into account. Napiwotzki thus recommends using pure hydrogen models if an LTE analysis of the Balmer lines is to be performed, which is what we are assuming here.

A more complex issue is posed by the discovery of trace abundances of elements heavier than helium - iron and other heavy metals, CNO, and Si in particular - in the atmospheres of most DA stars generally hotter than 50,000 K, in particular in the DAO white dwarfs. High dispersion spectra with the International Ultraviolet Explorer resulted in the detections of various ions in DA stars. It is impossible here to cite the large number of papers responsible for this discovery (see Holberg, Barstow \& Sion 1998). However, the discovery of unexpectedly-large opacities at the extreme ultraviolet (EUV) wavelengths measured with ROSAT and EUVE played a pivotal role (cf. Marsh et al. 1997; Vennes et al. 1997b). At the same time, Napiwotzki (1992) encountered a problem in fitting simultaneously the Balmer lines of hot, hydrogen-rich DA, DAO and H-rich planetary nebulae central stars; it now appears that elements heavier than helium need to be accounted for, in order to get correct 
fits and stellar parameters (Bergeron et al. 1993; Werner 1996). The sense of the problem is that the $T_{\text {eff }}$ value obtained by fitting the synthetic line profiles of a pure- $\mathrm{H}$ atmosphere is higher than the $T_{\text {eff }}$ obtained with additional heavy element opacities (Barstow et al. 2003b), at least for NLTE models. The problem is obviously complex, since a large number of radiatively-levitated ions might be contributing, but there is insufficient spectrophotometric information to identify these individually. The dependence of $\log g$ on the heavy element opacities may be more complicated.

It should be noted there presently also exists a significant discrepancy between $T_{\text {eff }}$ results obtained from the type of Balmer line analyses used in this paper and analogous methods used to fit the Lyman line profiles in very hot DA white dwarfs. Barstow et al. (2003a) have analyzed the Lyman line profiles of 16 DA white dwarfs observed with the Far Ultraviolet Spectroscopic Explorer (FUSE) and compared these results with temperature determined from the Balmer lines of the same stars. Specifically, it was found that marked systematic differences between the two methods occur for those stars with $T_{\text {eff }}>50,000 \mathrm{~K}$. The sense of this difference is that the Lyman $T_{\text {eff }}$ values are $\sim 10 \%$ higher than the corresponding Balmer values. At lower temperatures, the two methods are in very good accord.

The DA white dwarfs from the complete PG sample cover a wide range of effective temperatures including hot stars where NLTE effects on Balmer line profiles are important (Napiwotzki 1997), and cooler stars where energy transport by convection dominates. Even though NLTE model atmosphere codes are now becoming widely available (e.g., TLUSTY and SYNSPEC; Hubeny \& Lanz 1995), these codes are usually not able to handle convection satisfactorily. Our strategy was thus to adopt our LTE model atmosphere code (see BSL and references therein), which can handle convection reliably, up to an effective temperature where NLTE effects are still negligible and the atmospheres are completely radiative, and then switch to the TLUSTY and SYNSPEC packages to deal with NLTE effects at higher temperatures.

First, we need to ensure that at the branching point, both model atmosphere codes yield similar atmospheric structures and model spectra. I. Hubeny (private communication) kindly calculated for us LTE and NLTE pure hydrogen models and spectra for $T_{\text {eff }}>20,000 \mathrm{~K}$ using TLUSTY and SYNSPEC. In an experiment similar to that shown in Figure 3 of Napiwotzki et al. (1999), we fitted the Balmer lines of the LTE models with the NLTE spectra to derive the LTE corrections displayed in Figure 3; these represent the corrections that would need to be applied to the atmospheric parameters obtained under the assumption of LTE models. Our results are qualitatively in agreement with the results of Napiwotzki et al. (Fig. 3), although the effects in $\log g$ found at high effective temperatures are significantly smaller here (note that in Figure 3 of Napiwotzki et al. the offsets are magnified 3 times in both $T_{\text {eff }}$ 
and $\log g$ ). Thus, LTE models tend to overestimate both $T_{\text {eff }}$ and $\log g$, although the effects in $\log g$ are practically negligible $(\lesssim 0.03 \mathrm{dex})$. As illustrated in our Figure 3 , the onset of differences due to NLTE becomes significant about 40,000 K.

The next step was to fit our own grid of LTE model spectra with those calculated with TLUSTY and SYNSPEC under the assumption of LTE. Surprisingly, the differences in $T_{\text {eff }}$ and $\log g$ found initially were much larger than the LTE/NLTE differences observed in Figure 3! The origin of these differences was traced back to the use of different tables of Stark broadening for the hydrogen lines - our LTE models now make use of the extended calculations of Lemke (1997), as does SYNSPEC - to the details of the implementation of the Hummer \& Mihalas occupation probability formalism (Bergeron 1993), and more importantly, to the number of depth points and frequency points used in the model calculations. So even though NLTE effects are indeed important for the analysis of hotter DA stars (see Fig. 3), attention to details such as those listed above may be equally important.

After the differences between the LTE/TLUSTY code and our own LTE code were understood and resolved, the LTE synthetic spectra obtained from both codes agreed to better than $1 \%$ in $T_{\text {eff }}$, and $0.02 \mathrm{dex}$ in $\log g$, from $T_{\text {eff }}=20,000 \mathrm{~K}$ to $90,000 \mathrm{~K}$. Thus, the effective temperature at which the two model grids were matched was set at 20,000 K where the convective flux is zero, and where the LTE approximation holds. The NLTE switch in TLUSTY was then turned on to calculate model spectra above $T_{\text {eff }}=20,000 \mathrm{~K}$, while our own LTE code was used to calculate cooler (convective) models. Our complete pure hydrogen model grid covers a range between $T_{\text {eff }}=1500 \mathrm{~K}$ and $140,000 \mathrm{~K}$ by steps of $500 \mathrm{~K}$ at low temperatures $\left(T_{\text {eff }}<17,000 \mathrm{~K}\right)$ and $5000 \mathrm{~K}$ at high temperatures $\left(T_{\text {eff }}>20,000 \mathrm{~K}\right)$, and a range in $\log g$ between 6.5 and 9.5 by steps of 0.5 dex (steps of 0.25 dex were used between $8000 \mathrm{~K}$ and 17,000 $\mathrm{K}$ where Balmer lines reach their maxima). We thus end up with a homogeneous model grid that includes consistently NLTE effects as well as convective energy transport, and completely covers the range of parameters in the PG sample.

\subsection{Fitting Technique}

Our fitting technique is an improved version of that used by BSL. The first step is to normalize the flux from an individual line, in both observed and model spectra, to a continuum set to unity at a fixed distance from the line center. The comparison with model spectra, which are convolved with the appropriate Gaussian instrumental profile $(3,6$, or $9 \AA)$, is then carried out in terms of these line shapes only. The most sensitive aspect of this fitting technique is to define the continuum of the observed spectra. Here we rely on the procedure outlined in Bergeron et al. (1995) where the observed spectrum is fitted with 
several pseudo-Gaussian profiles (see also Saffer et al. 1989) using the nonlinear least-squares method of Levenberg-Marquardt (Press et al. 1986). The normal points are then fixed at the points defined by this smooth function. As discussed by Bergeron et al. (1995), this method is much more accurate when a glitch is present in the spectrum at the location where the continuum is set. It also provides a precise value of the line center which can be corrected to the laboratory wavelength.

This procedure is quite reliable in the temperature range where the Balmer lines are strong and can be approximated as a sum of pseudo-Gaussian profiles $\left(16,000 \gtrsim T_{\text {eff }} \gtrsim 9000 \mathrm{~K}\right)$. Outside this temperature range the method becomes more unstable when the continuum between the lower Balmer lines becomes linear, or when the higher Balmer lines vanish. There, instead of using pseudo-Gaussian profiles, we rely on theoretical spectra to reproduce the observed spectrum, including a wavelength shift, a zero point, as well as several order terms in $\lambda$ (up to $\lambda^{6}$ ). The normal points are then fixed at the points defined by this smooth model fit. Note that the values of $\log g$ and $T_{\text {eff }}$ at this stage are meaningless since too many fitting parameters are used, and the model just serves as a smooth fitting function to define the continuum of the observed spectrum. Examples of continuum fitting using the two procedures described above are shown in Figure 4.

Once the Balmer lines are normalized to a continuum set to unity, we use our grid of model spectra to determine $T_{\text {eff }}$ and $\log g$ in terms of these normalized profiles only. Our minimization technique again relies on the nonlinear least-squares method of LevenbergMarquardt, which is based on a steepest descent method. We consider here only $\mathrm{H} \beta$ to $\mathrm{H} 8$ in the fitting procedure. For cases where the red portion of the spectrum is contaminated by an unresolved companion, we neglect $\mathrm{H} \beta$ in the fitting procedure (PG 0014+098, 0805+655, $0933+026,1026+002,1037+512,1049+103,1314+294,1443+337,1622+324,1646+062)$, and in some cases even $\mathrm{H} \gamma(0004+061,0824+289,0950+186,1001+204,1210+464$, and $1643+144)$.

As is well known, when the effective temperature of the white dwarf is close to the region where the equivalent widths of the Balmer lines reach a maximum $\left(T_{\text {eff }} \sim 13,500 \mathrm{~K}\right.$; see Fig. 4 of Bergeron et al. 1995), two solutions are possible, one on each side of the maximum. Even though the optical spectra are not spectrophotometric in the sense that their slopes are accurate enough to serve as a precise temperature indicator, they can still be used to discriminate between the cool and the hot solutions. Hence for such stars in our sample, we examine the two solutions obtained by using a cold and a hot seed, compare the model spectrum with the observed spectrum, both normalized at $4600 \AA$, and pick the solution that best reproduces the observed energy distribution.

Effective temperatures and surface gravities are obtained in this manner for all 347 
DA stars in the PG sample. A small number of objects in our sample have been analyzed in greater detail in the literature than within our current framework, in which cases we substitute our $T_{\text {eff }}$ and $\log g$ values with those taken from these studies. This is the case for the magnetic white dwarfs PG 1031+234 (Schmidt et al. 1986), 1220+234 (Liebert et al. 2003), 1533-057 (Liebert et al. 1985), and 1658+441 (Schmidt et al. 1992), the DAO stars $0134+181,0823+317,0834+501,0846+249,1202+608,1210+533,1214+268$, 1252+378, and 1305-017 (Bergeron et al. 1994), the unresolved double degenerate systems PG 0945+246 (LB 11146, Liebert et al. 1993) and 1115+166 (Bergeron \& Liebert 2002).

\subsection{Results}

The atmospheric parameter determinations for the 347 DA stars from the complete PG sample are reported in Table 1; the values in parentheses represent the internal errors of the fitting technique for $T_{\text {eff }}$ and $\log g$. These represent only the formal uncertainties of the fitted atmospheric parameters obtained from the covariance matrix (see BSL and Press et al. 1986 for details). Sample fits covering the temperature range of our sample are displayed in Figure 5. In addition, we provide in Table 2 the atmospheric parameters of several DA stars in the PG survey that were also observed but which are not part of the complete sample.

Other quantities in Table 1, derived from the evolutionary white dwarf models, include the stellar mass $(M)$ and uncertainty, the absolute visual magnitude $\left(M_{V}\right)$, the luminosity $(L)$, the $V$ magnitude, the distance $\left(D_{\mathrm{pc}}\right)$, the $1 / V_{\max }$ weighting $\left(\mathrm{pc}^{-3}\right)$, and the log of the cooling time $(\log \tau)$.

The $V$ magnitudes are important in estimating the distances of the stars. There exists published non-photographic photometry for 291 of the PG DA stars. For these we have determined a mean $V$ band magnitude using broadband $V$ or equivalent Strömgren $y$ magnitudes from McCook \& Sion (1999). In some cases obviously discrepant magnitudes were excluded. In general Palomar multi-channel spectrophotometry (Oke 1969) was not used, unless it was the only available source of photometry. In those cases the multi-channel $v$ (Greenstein 1976) was converted to $V$ using the relation $V=v+0.07$. For $12 \mathrm{PG}$ stars we were able to use DR 1 or DR 2 Sloan Digital Sky Survey (SDSS) photometry to determine an equivalent $V$ from the transformation of the SDSS $g$ and $r$ band magnitudes taken from Smith et al. (2002); these are noted in Table 2. For 43 stars only the original PG photographic $m_{b}$ magnitudes were available. For these stars we estimated an effective $V$ magnitude by using the larger sample of PG stars to form a correlation between the spectroscopic $M_{V}$ and the pseudo color $m_{b}-V$. As expected there is a substantial dispersion in the $m_{b}$ values (standard deviation, 0.477 magnitudes) and consequently our $V$ band estimates for these 
stars are quite uncertain. Values for these stars are given only to tenths of the magnitude, and they are also noted in Table 2 .

Since the calculations of cooling times require the use of evolutionary models for white dwarfs, it is necessary to make an assumption about the thickness of the outer hydrogen layer mass. While the weight of evidence now is that most DA white dwarfs have "thick" outer hydrogen layers - i.e., "evolutionary" layer masses of the order $q(\mathrm{H}) \equiv M_{\mathrm{H}} / M_{\star}=10^{-4} M_{\odot}-$ there is still reason to believe that a minority of DA stars, in particular those becoming DB stars below the 30,000-45,000 K DB gap, have much thinner outer layers. Layer masses one or more orders of magnitude less than the evolutionary value are better modeled using the assumption of zero hydrogen layer mass, as was assumed in the BSL study. It is well known that the assumption of an evolutionary $\mathrm{H}$ layer results in the determination of a larger mass, and the effect is greater for the hotter and/or lower mass stars.

Since the carbon-core models of Wood (1995) with thick hydrogen layers of $q(\mathrm{H})=10^{-4}$ extend to much higher effective temperatures than those of Wood (1990) with no hydrogen layers, we use the former models throughout. Low mass white dwarfs, however, are likely to be composed of helium cores, for which we rely instead on the evolutionary helium-core models of Althaus et al. (2001). To illustrate the parameter space used for the interpolation and to show also how these different evolutionary models match in terms of mass, we plot in Figure 6 the $T_{\text {eff }}$ and $\log g$ values for the complete PG sample together with the evolutionary models of Wood (1995) and Althaus et al. (2001). Unfortunately, the Althaus et al. models go up to only $0.406 M_{\odot}$. Since there appears to be a paucity of stars near $0.45 M_{\odot}$ (see Fig. 6 and also our mass distribution below), we fix arbitrarily our mass cutoff between carbon- and helium-core models at $0.46 M_{\odot}$. This ensures that the inferred masses for the helium-core white dwarfs are still reasonably well extrapolated, while remaining below the masses obtained for the white dwarfs with slightly larger values of $\log g$ but inferred from the carbon-core models of Wood.

The NLTE effects on the determination of white dwarf atmospheric parameters can be estimated by analyzing all stars in our sample with LTE models. The comparison between LTE and NLTE results is displayed in Figure 7. The results show that NLTE effects are always small for the analysis of DA stars, in agreement with the conclusions reached by Napiwotzki (1997). The general trend is that LTE solutions tend to overestimate both the effective temperatures and the surface gravities. In some instances, the differences in $\log g$ are significantly larger than those inferred from Figure 3 using models only. These correspond to the white dwarfs that show the so-called "Balmer line problem" first discussed by Napiwotzki (1992), for which neither the LTE nor the NLTE pure hydrogen models provide a satisfactory fit to the observed hydrogen line profiles. 
As discussed many places in the literature (see, e.g., BSL), the internal errors of our fitting technique given in Table 1 can be made arbitrarily small given that the signal-tonoise ratio is high, and that the model spectra reproduce the observed data in detail. The true error budget, however, is dominated by the external uncertainties originating from the flux calibration in particular. During the course of this project, we have obtained multiple observations for many stars in order to increase the overall signal-to-noise ratio of the sample, and to make the spectroscopic data set as homogeneous as possible. As such, we ended up with multiple (two spectra or more) observations for 126 white dwarfs in our sample, for a total of 284 spectra. Of course for each star, we always pick the best spectrum in the final analysis. Since all spectra have been obtained independently by different observers, with an independent reduction, etc., these spectra can be used to get an estimate of the external error of the atmospheric parameter determinations. We thus fitted all 284 spectra using our fitting technique, and calculated the average parameters and standard deviations for each star, which are displayed in Figure 8 as a function of $T_{\text {eff }}$. The standard deviations in $T_{\text {eff }}$ are divided by the average temperature for each star to obtain a distribution that is largely independent of $T_{\text {eff }}$. We also show in Figure 8 the average standard deviations of each atmospheric parameter, $0.038 \mathrm{dex}$ in $\log g$ and $1.2 \%$ in $T_{\text {eff }}$, which we adopt as a measure of the external error of our fitting procedure. Note that these are even conservative estimates since each time, our best spectrum is compared with admittedly worse spectra. Further note that we do not take into account possible systematic errors related to the model atmospheres themselves.

\subsection{Comparison with Other Investigations}

As discussed in the introduction, Balmer line analyses of other large samples of DA stars have been conducted by various investigators. In general these analyses have relied on Balmer line fitting techniques similar to those described in this paper but have employed independent observational data and independently computed model grids. It is therefore important to investigate the degree of consistency between our $T_{\text {eff }}$ and $\log g$ results and these unrelated data sets. Such comparisons also allow an assessment of the role of external systematic errors between the various data sets, models and fitting techniques.

In Figure 9 we compare our $T_{\text {eff }}$ and $\log g$ determinations for PG stars which we have in common with the data sets of Finley et al. (1997); Vennes et al. (1997b); Marsh et al. (1997); Homeier et al. (1998) and Koester et al. (2001), respectively. In each case we plot

the quantities $T_{\text {others }}-T_{\mathrm{PG}}$ and $\log g_{\text {others }}-\log g_{\mathrm{PG}}$ vs $T_{\mathrm{PG}}$, where the subscripts refer to the above independent data sets and our PG data set, respectively. In order to estimate 
any systematic offsets in temperature and gravity between our results and others, we have calculated unweighted averages of the data in Figure 9. Unweighted averages were used because it is clear in each case that both the uncertainties in both the temperature and gravity are large with respect to the true level of mutual uncertainty for individual stars.

\subsubsection{Finley et al. Data}

Finley et al. (1997) determined the effective temperatures and surface gravities of 174 DA stars having $T_{\text {eff }}$ greater than $25,000 \mathrm{~K}$. These stars were selected from a variety of catalog sources including DA stars detected in the ROSAT and EUVE all-sky EUV surveys. The analysis of the temperatures and gravities is based on pure hydrogen LTE atmospheres derived from the grids of Koester. The masses are calculated from the thick models of Wood (1995). There are 62 stars in common between our PG sample and those of Finley et al. (1997). In Figure 9 we show the comparison of the Finley et al. $T_{\text {eff }}$ and $\log g$ determinations with respect to our results. The Finley et al. data are, on average, $1.7 \%$ lower in temperature and 0.06 dex lower in gravity than our results.

\subsubsection{Vennes et al. Data}

Vennes (1999) describes the results for a purely EUV-selected sample of 141 DA stars. The full $T_{\text {eff }}$ and $\log g$ results for this sample are variously contained in Vennes et al. (1996, 1997a,b), and Vennes (1999). Due to the EUV-selected nature of this sample the $T_{\text {eff }}$ values of all stars are effectively in excess of $25,000 \mathrm{~K}$. The analysis of the temperatures and gravities is based on pure hydrogen LTE atmospheres derived from the grids of Vennes (1992). Masses are calculated for both the thick and thin models of Wood (1995). There are 17 stars in common between our PG sample and those of Vennes et al. (1997b). In Figure 9 we show the comparison of the Vennes $T_{\text {eff }}$ and $\log g$ determinations with respect to our results. The Vennes et al. data are on average $0.6 \%$ lower in temperature and 0.03 dex higher in gravity than our results.

\subsubsection{Marsh et al. Data}

Marsh et al. (1997) describe the results for a purely EUV-selected sample of 89 DA stars. As with the Vennes sample, all stars are effectively in excess of 25,000 K. The analysis of the temperatures and gravities is based on pure hydrogen LTE atmospheres derived from 
the grids of Koester, while the masses are calculated from the thick models of Wood (1995). There are 16 stars in common between our PG sample and those of Marsh et al. (1997). In Figure 9 we show the comparison of the Marsh et al. $T_{\text {eff }}$ and $\log g$ determinations with respect to our results. The Marsh et al. data, which show the most variance of all data sets, are on average $3.3 \%$ lower in temperature and 0.10 dex lower in gravity than our results.

\subsubsection{Homeier et al. Data}

Homeier et al. (1998) describe the results for an initial sample of 80 DA stars drawn from the Hamburg Quasar Survey, an objective prism survey of at high northern galactic latitudes. The Homeier et al. sample covers the temperature range above 10,000 K. The analysis of the temperatures and gravities is based on pure hydrogen LTE atmospheres derived from the grids of Koester while the masses are calculated from the thick hydrogen models of Wood (1995). There are 9 stars in common between our PG sample and those of Homeier et al. In Figure 9 we show the comparison of the Homeier $T_{\text {eff }}$ and $\log g$ determinations with respect to our results. The Homeier et al. data are on average $0.3 \%$ higher in temperature and 0.08 dex lower in gravity than our results.

\subsubsection{Koester et al. Data}

The SPY project is a large, general observational program to find close binaries from several white dwarf surveys, mainly as potential progenitors for SNIa (Napiwotzki et a. 2001). Koester et al. (2001) analyze stars from the SPY project in a temperature range between about 8,000 and 30,000 K. There are 17 stars in common with our sample. In Figure 9 we show the same comparisons with respect to our results. The Koester et al. data are on average $0.6 \%$ higher in temperature and 0.08 dex lower in gravity than our results.

\section{LUMINOSITY, MASS AND OTHER DISTRIBUTION FUNCTIONS}

In Liebert \& Bergeron (1995) and especially in Bergeron, Leggett, \& Ruiz (2001), the importance of combining the mass and luminosity functions (MFs, LFs) into one distribution function for the comparison of cooling times is emphasized. The primary parameter we will be seeking is the formation rate of white dwarfs in the recent history of the local Galactic

disk. However, for comparison with the traditional, published LFs, we present first the derived LF for all 347 stars. 


\subsection{A Comparison of Luminosity Functions}

The LF was calculated using the method of Green (1980) and Fleming et al. (1986). Briefly, given the limiting magnitude of the particular field in which a given object was found, we let $V_{\max }$ be the volume defined by the maximum distance at which a given object would still appear in the sample. To correct for the nonuniform distribution of stars (i.e. the Galactic disk scale height), we define $d V^{z}=\exp \left(-z / z_{0}\right) d V$, where $z_{0}$ is the assumed scale height. Then each star's contribution to the local space density is $1 / V_{\max }$.

In Figure 10 the visual luminosity function of all DA stars in the complete PG sample is presented in half-magnitude bins, assuming a scale height for the Galaxy of $z_{0}=250 \mathrm{pc}$ (as did Fleming et al. 1986). The dotted line represents the results of Fleming et al. (1986), and the dashed line that for the KUV sample of Darling (1994) to be discussed later. Note that the color correction factors given in Table 2 of Fleming et al. are not included in our calculations. To within the errors, which are of order $30 \%$ and larger in a $1 / V_{\max }$ calculation, the two earlier and present PG LFs are indistinguishable. The total number of DA white

dwarfs per $1000 \mathrm{pc}^{-3}$ is again $0.50 \pm 0.05$ for $M_{V}<12.75$. The close agreement of the two determinations might seem surprising, given (1) the appreciable number of misclassified white dwarfs and subdwarfs in the PG (see $\S 2.1$ ), and (2) the crudeness with which Fleming et al. (1986) were able to estimate $T_{\text {eff }}$ values for each star using a heterogeneous set of pre-CCD era spectrophotometry. The method is one-dimensional since a monotonic relation between $M_{V}$ and $T_{\text {eff }}$ assumes basically that all white dwarfs have the same mass. Moreover, as we shall show, the PG sample would need a substantial correction for incompleteness at the redder colors (fainter $M_{V}$ bins). The LF presented here is therefore a crude, first estimate of a two-dimensional function.

\subsection{The Mass Distribution}

The entire mass distribution as a function of $T_{\text {eff }}$ is shown in Figure 11, together with evolutionary isochrones discussed further below. Below $T_{\text {eff }} \sim 13,000 \mathrm{~K}\left(\log T_{\text {eff }}=4.11\right)$, the atmospheres of DA stars become convective, and there is the suspicion that the high masses inferred from spectroscopy below $\sim 12,000 \mathrm{~K}$ are actually a measure of the presence of helium brought to the surface by the hydrogen convection zone (Bergeron et al. 1990, BSL). As in BSL, our strategy is thus to exclude all stars below 13,000 K from the following discussion. The resulting gravity and mass distributions for the 297 DA stars above 13,000 K are shown in Figure 12. A bin size of 0.1 dex was used for the gravity distribution, which has a mean of 7.885. As the mass distribution is the primary parameter of interest, we do not discuss the gravity distribution further. 
The mass distribution shown is constructed from a binning of the 297 masses over the range 0.2 to $1.2 M_{\odot}$, using a bin size of $0.025 M_{\odot}$. This is a significantly finer binning than the commonly employed $0.05 M_{\odot}$ used by many previous studies having smaller sample sizes. To within the limits of poisson statistics, the complete PG mass distribution can be well characterized by three primary components with assumed Gaussian profiles. The primary peak is centered on $0.565 M_{\odot}$, with a FWHM of $0.188 M_{\odot}$. At the lowest masses there is a peak centered on $0.403 M_{\odot}$, with a FWHM of $0.055 M_{\odot}$. Finally, we tentatively identify a broad, high-mass component centered on $0.780 M_{\odot}$ with a FWHM of $0.255 M_{\odot}$. This, unfortunately, appears to overlap considerably in mass with the primary $0.6 M_{\odot}$ component, if the assumption of a Gaussian shape is correct. As we shall discuss, this assumption does not predict the correct number of stars with masses $>1.0 M_{\odot}$. The respective contributions of the $0.4 M_{\odot}$, the $0.6 M_{\odot}$ and the $>0.8 M_{\odot}$ components are $8 \%, 76 \%$ and $16 \%$ of the unweighted sample.

The peak just below $0.6 M_{\odot}$ was first pointed out by Koester, Schulz, \& Weidemann (1979), and the sharpness of this peak is now well documented from several modern studies already cited (Bergeron et al. 1992; Bragaglia, Renzini, \& Bergeron 1995; Marsh et al. 1997; Vennes et al. 1997b). In the entire PG sample the peak is located at $0.572 M_{\odot}$, significantly less than our mean sample mass of $0.62 M_{\odot}$. (This is due to the fact that there are more stars in the high mass component than in the low mass component.) The intrinsic width of this peak should be slightly less than the $0.188 M_{\odot}$ FWHM, due to the broadening introduced by the inherent uncertainties in the estimated masses. However, the dominant central component is effectively resolved.

Assuming that all of the stars in the central peak represent single white dwarfs, and the number of undiscovered double degenerates is low, then approximately $76 \%$ of the PG white dwarfs fall into a fairly narrow range of masses. Considering the stars within one standard deviation of the peak, the mean age and temperature of these stars is $7.5 \times 10^{7}$ years and 27,400 K, respectively. The corresponding range of progenitor main sequence masses of these stars is also fairly restricted. Using the revised initial-final mass relation of Weidemann (2000), this range of white dwarf masses would correspond to main sequence progenitor stars with masses between approximately 1.0 to $2.5 M_{\odot}$, essentially spectral types G2 to A0. If the initial-final mass relation were strictly monotonic with no dispersion, the central peak should have a sharp low-mass cutoff - see Weidemann \& Yuan (1989). The relative symmetry of the wings suggests that there is appreciable dispersion.

The low mass peak consists of He-core white dwarfs, formed when the He-core burning phase is truncated during a common-envelope phase in close binary systems. As predicted in BSL, these stars turn out to be close binaries, usually double degenerate systems (Marsh et 
al. 1995). These must be white dwarfs with cores composed of helium, whose envelopes were removed in the red giant phase before the core mass reached the $0.45-0.50 M_{\odot}$ necessary for core-helium ignition Iben \& Renzini (1984). Note that the minimum between the two peaks is in this interval, as would be expected. The lowest mass case is PG $1101+364$ at 0.32 $M_{\odot}$. In our analysis we implicitly assume that the spectra of all He-core white dwarfs are dominated by a single luminous degenerate star. The existence of significant spectroscopic or photometric contributions from a secondary degenerate star can bias our mass and distance estimates for such stars.

There also exists a distinct high mass shoulder next to the central peak, which we are able to characterize as a much broader Gaussian with a centroid of $0.780 M_{\odot}$ and a FWHM of $0.255 M_{\odot}$. Computing means for the stars with in $\pm 0.1 M_{\odot}$ of the centroid yields characteristic ages and temperatures of $1.1 \times 10^{8}$ years and $25,500 \mathrm{~K}$, considerably older and cooler then the central peak. If these result from single star evolution, the progenitors would have had a mean mass of about $3.8 M_{\odot}$, but there appear to be somewhat more of them than modeling with a standard initial mass function would predict as single-star evolution (Weidemann \& Yuan 1989). There may also exist a high mass excess of stars between 1.0 and $1.2 M_{\odot}$. Several authors (Vennes et al. 1997b; Vennes 1999; Marsh et al. 1997) have suggested the existence of one or more high mass peaks in this range. However, these were based primarily on EUV selected samples of hot DA stars. While there well may be selection effects with these samples that are responsible for an apparent excess of high mass stars, in our sample we can use the Gaussian tail of the high mass shoulder to estimate the expected number of stars in the range 1.0 to $1.2 M_{\odot}$. Extrapolating the high mass shoulder indicates that there should be less than one star in our sample with a mass this high, in contrast there are eight such stars. The few such objects expected from single-star evolution with masses above about $1.2 M_{\odot}$ might have cores composed of oxygen-neon-magnesium $(\mathrm{O}-\mathrm{Ne}-\mathrm{Mg})$, the primary products of the nucleosynthesis of ${ }^{12} \mathrm{C}$.

It has been been suggested by several authors, due to this apparent excess of massive stars, that a substantial fraction of the $0.8-1.35 M_{\odot}$ result from close binary evolution, in particular mergers white dwarfs of double helium ( $\mathrm{He}-\mathrm{He})$, helium-carbon-oxygen $(\mathrm{He}-\mathrm{CO})$, and double carbon-oxygen $(\mathrm{CO}-\mathrm{CO})$ core compositions. This role was in fact suggested by Marsh et al. (1997) and Vennes (1999), among others.

The mass distribution discussed above, however, is biased substantially in a magnitudelimited survey like PG, due to the corresponding variation in the radius of the white dwarfs. The search radius to a given limiting magnitude is proportional to $R$, so the search volume is proportional to $R^{3}$ (Shipman 1972; Liebert \& Bergeron 1995). The search volumes available for each star differ for this and other reasons, but the $1 / V_{\max }$ weighting corrects for all of 
these.

Hence we show in Figure 13 the mass distribution corrected for the actual volumes searched. This distribution should display the true space densities of white dwarfs of various mass intervals. For the three mass components identified earlier, the total space densities of DA stars with $T_{\text {eff }} \gtrsim 13,000 \mathrm{~K}$ are $4.52 \pm 0.03 \times 10^{-6} \mathrm{pc}^{-3}$ for the $0.4 M_{\odot}$ component, $1.183 \pm 0.75 \times 10^{-4} \mathrm{pc}^{-3}$ for the $0.6 M_{\odot}$ "peak" component, and a surprising $3.53 \pm 0.22 \times 10^{-5}$ $\mathrm{pc}^{-3}$ for the $>0.8 M_{\odot}$ massive component. The total space density of DA white dwarfs based on this PG sample is $1.58 \times 10^{-4} \mathrm{pc}^{-3}$, while the three mass components account for $3 \%, 75 \%$, and $22.0 \%$ of the hot DA sample, respectively. Because of the absence of lower temperature, lower luminosity stars, this total density is considerably lower than that estimated in the previous subsection from the LF for $M_{V}<12.75$.

The shape of Figure 13 suggests that any high mass component may split into sharper components centered near $0.9 M_{\odot}$ and beyond $1.0 M_{\odot}$. The latter accounts for a space density of $1.02 \times 10^{-5} \mathrm{pc}^{-3}$, or $6.4 \%$ of the total DA white dwarf density! We have already noted that such a separate component of $>1 M_{\odot}$ white dwarfs exists has again previously been identified in their EUV-selected samples by such authors as Marsh et al. (1997) and Vennes (1999).

These PG DA white dwarf density estimates are just lower limits, however. It is necessary to correct for the incompleteness of the survey, for white dwarfs hidden by luminous nondegenerate companions, for stars that are actually double degenerate systems, and for DB/DO stars if one desires the total space density of hot white dwarfs. Most importantly, as we shall document in the next section, the consideration of the space densities of different components of white dwarfs gives an incomplete, and probably misleading, picture. One must fold in the evolution times for stars of different masses, in order to calculate the formation rates of each group of stars.

\subsection{The Luminosity Function Revisited: The Three Mass Components}

Since the $0.4 M_{\odot}, 0.6 M_{\odot}$, and $>0.8 M_{\odot}$ components may represent, at least in part, separate paths of single and binary stellar evolution through the white dwarf phase, it is appropriate to break up the LF of Figure 10 into (at least) three components. This largely removes the mass dependence on the LF, and leads to insight into the selection effects due to magnitude and color (temperature) limits. For now we will retain the $>0.8 M_{\odot}$ component intact. Small numbers would make it difficult to subdivide the component.

In Figure 14, as expected, it is seen that the $0.6 M_{\odot}$ peak contributes the most to the 
white dwarf space density. The $>0.8 M_{\odot}$ component rises much more sharply at lower luminosities. While contributing very little at $M_{V}<10.25$, near $M_{V} \sim 13$ its contribution comes close to matching that of the $0.6 M_{\odot}$ LF. Some understanding of this behavior comes from the very different cooling rate for massive white dwarfs, compared to the norm. At highest luminosities, these cool more rapidly due to neutrino losses from their dense cores. At lower luminosities, however, they cool much more slowly: they have much greater internal energy per unit volume in the core, but smaller surface areas from which to radiate it away. So the massive stars evolve over this interval from cooling more rapidly to cooling considerably more slowly than the $0.6 M_{\odot}$ peak stars. As to their identification in the PG Survey, their cooling times offset the selection effect due to the smaller survey volume in which they may be found.

Very contrasting in behavior is the distribution of the $0.4 M_{\odot}$ component. This LF contributes down to $M_{V} \sim 11$, but nothing fainter than that magnitude. These will linger as hot stars since their low density interiors lose little energy to neutrinos. A selection effect, however, is very important: at $M_{V}>11$, they are luminous due to their large radii, but the $T_{\text {eff }}$ fall below the $10,000 \mathrm{~K}$ limit due to the UV-excess selection of the PG Survey. Their rapid evolution time offsets their larger survey volumes in the PG selection.

\subsection{The Mass and Luminosity Distributions vs. Temperature}

Crossing the mass range in Figure 11 are evolutionary isochrones with cooling times of $\log \tau=5.5-9.5$ years obtained from the carbon-core models of Wood (1995, solid lines). This is not appropriate for masses much below $0.5 M_{\odot}$, and we will return to this point shortly. It is seen that completeness certainly stops near $\log T_{\text {eff }}$ of 4.0 , where the $0.4 M_{\odot}$ stars are near $M_{V} \sim 11$ but the $>0.8 M_{\odot}$ stars approach $M_{V} \sim 13$, as we saw in Figure 14 . The faster neutrino-cooling phase of the high mass stars is brief, and these stars cool more slowly

at cooling times $\tau>10^{6}$ years. They cool much more slowly by $10^{9}$ years. The 0.4 and 0.6 $M_{\odot}$ show sharp peaks in two dimensions, while the distribution of high mass stars is again more extended.

An alternative way of highlighting similar themes is to plot the absolute visual magnitude $M_{V}$ vs. $\log T_{\text {eff }}$. This is shown in Figure 15. Diagonal solid curves from the upper left to the lower right are lines of constant mass from the Wood models, from $0.4 M_{\odot}$ (top) to 1.2 $M_{\odot}$ in intervals of $0.2 M_{\odot}$, as labeled in the lower left of the figure. The dashed curves are evolutionary tracks from He interiors of Althaus et al. (2001), with the masses labeled in the upper right of the figure. Crossing the mass lines are isochrones with log cooling times for 0.5 dex intervals from 5.5 to 9.0. Note that the helium track isochrones for 7.5 and earlier are 
too luminous to fall in this plot. Here the slope of the isochrones due to the slower cooling of the massive stars turns nearly horizontal! Incompleteness near $T_{\text {eff }} \sim 10,000 \mathrm{~K}$ clearly imposes a sharp cutoff in $M_{V}$ as a function of mass.

\section{COMPLETENESS OF THE PG SAMPLE}

PG was a photographic survey performed in the 1970s using the Palomar 46-cm Schmidt telescope. The detector was baked IIa-O film. The fields were circular in shape, and overlapped by $<10 \%$. A double, offset $m_{u}$ and $m_{b}$ exposure was made on each field. Point sources were scanned with a microdensitometer. Selection of "UV-excess" candidates was made on the basis of a photographic $m_{b}$ limiting magnitude (near 16.2) and a $m_{u}-m_{b}$ color cut. Photoelectric photometry was obtained of a few standards in each PG field in order to calibrate to a photometric system. the accuracy of the $m_{b}$ values was believed to be $0.29 \mathrm{mag}$. The color cut was applied to identify candidates having $m_{u}-m_{b}<-0.46$, but the dispersion in $m_{u}-m_{b}$ was modelled as a Gaussian with $\sigma=0.38$ mag. Star-count modelling tied to the local standards showed the $m_{b}$ limit varied from field to field, and such observations were necessary to determine it for each field. Moreover, followup spectroscopy showed that the actual $m_{u}-m_{b}$ cut also varied considerably from field to field, as was evident in particular by large variation in the number of weak-lined main sequence stars extracted as candidates, because of the large uncertainties, and the realized $m_{u}-m_{b}$ cut varied appreciable from field to field.

The preceding paragraph is a brief summary of the details provided in Green et al. (1986), to give the reader a sense of how this three-decades-old survey was carried out. Some $46.8 \%$ of the survey area was covered by two exposures, and various tests suggested to the authors that the overall completeness to the specified magnitude and color limits was about $84 \%$, although others (see below) have argued that this number is too optimistic. In estimating the luminosity function, Fleming et al. (1986) attempted a color incompleteness correction which increased with $M_{V}$ (see their Table 2). We shall argue below, however, that this correction was probably too small for the fainter $M_{V}$ bins.

In the following decades, deeper surveys of fields overlapping the PG were performed, so that subsequent authors could evaluate the PG completeness. The Edinburgh-Cape Blue Object Survey also targetted the North Galactic Cap at Galactic latitude $|b|>30^{\circ}$, to a fainter limiting magnitude of $m_{b}=16.5$, and to somewhat redder stars. Kilkenny et al. (1997) concluded that the completeness of the PG averaged $84 \%$ relative to other surveys and compilations, which could themselves be incomplete. The area of overlap is not stated in the paper. These authors appeared to dispute the much less optimistic conclusions of 
Goldschmidt et al. (1992), who claimed a surface density of quasars a factor of 3.4 times higher than that found in the PG. The Goldschmidt group appeared to use an appreciablyfainter $m_{b}$ limit, which could make a large difference since the surface density of UV-excess quasars is known to rise sharply at $B \sim 16-17$.

Darling (1994) - see also Wegner \& Darling (1994) - did a similar comparison of the Kiso Survey (Noguchi et al. 1980; Kondo et al. 1984) with the PG. The Kiso Survey used $m_{u}$ and $m_{g}$ photographic colors, with 600 square degrees of overlap. Darling (1994) estimated the completeness of PG to be $57.5 \%$ for white dwarfs to the stated magnitude limits of the PG (though only $50 \%$ for quasars). Using his spectroscopically-identified sample of hot white dwarfs, Darling (1994) constructed a LF from the Kiso or KUV sample, shown in Figure 10. Wegner \& Darling (1994) stated that "On the whole, the luminosity function derived compares relatively well with that from the PG Survey (Fleming et al. 1986)." In fact, the KUV LF has significantly more stars than PG in the bins $M_{V} 10.5$ to 13.0, with the PG appearing to become incomplete by a factor of 4 in the 12.0 and the two fainter magnitude bins.

It has to be noted that, while spectra had been obtained to classify the DA white dwarfs of the Darling (1994) KUV sample, the assignment of $M_{V}$ values for individual stars was not based on the spectroscopic line-fitting method utilized here. Rather, an $M_{V}$ vs. $m_{u}-m_{g}$ relation was determined from $B-V$ photometry of a subset of the sample - the $M_{V}$ - color index or $M_{V}(\mathrm{CI})$ relation. The $B-V$ vs. $m_{u}-m_{g}$ relation had a very large dispersion - see Figure 4.1 of Darling (1994) - and necessarily so did the $M_{V}$ estimates.

We have been able to assess the dispersion and look for systematic error in the Darling (1994) $M_{V}$ estimates using KUV stars for which accurate CCD spectra are available - largely due to observation by one of us (P.B.), using the same Steward $2.3 \mathrm{~m}$ telescope and spectrograph. Excluding four DA $+\mathrm{M}$ stars for which the CI might be corrupted by the companion, $67 \mathrm{KUV}$ stars can be used to compare the spectroscopic line-fitting luminosities $M_{V}$ (sp) with $M_{V}(\mathrm{CI})$. The mean difference in $M_{V}(\mathrm{sp})-M_{V}(\mathrm{CI})$ is -0.526 , and a linear fit yields a relation of the form $M_{V}(\mathrm{sp})=-3.118+0.242 M_{V}(\mathrm{CI})$. Part of the growing difference between the KUV and PG LFs as $f\left(M_{V}\right)$ in Figure 10 is understandable from the systematic offset in $M_{V}$ between the two sets of determinations. However, this offset varies from from about -1 at $M_{V}(\mathrm{sp})=+9$ to 0 at $M_{V}(\mathrm{sp})=+13$. Since the KUV survey includes a redder color limit than $\mathrm{PG}$, it is clear that the latter becomes increasingly incomplete for the fainter magnitudes (redder colors), due to the large dispersion in the $m_{u}-m_{b}$ color measurements. In the following section, we present an additional argument. However, the offset in $M_{V}$ between the two surveys makes the KUV sample difficult to deal with for deriving a completeness correction for the bluer, hotter PG stars. 
The EUVE and ROSAT all-sky surveys detected only hotter white dwarfs, generally with $T_{\text {eff }}>25,000 \mathrm{~K}$. We have found that 18 EUVE sources, listed in Vennes et al. (1997b) and Vennes (1999) (but including ROSAT detections), were also found in PG. The EUVE and ROSAT surveys detected many white dwarfs with $B$ magnitudes fainter than the PG limit, which makes this a good source for testing the incompleteness in PG for hot white dwarfs - i.e. too blue for the $m_{u}-m_{b}$ color dispersion to be a significant problem. Rather, this is a cleaner test of the magnitude limit of the survey. Note that the application of this test does not require the assumption that the EUV surveys are 100\% complete in detecting white dwarfs to a particular flux level.

The 18 EUV-detected sources listed in the above references have PG (1950) designations of $0102+095,0136+251,0216+143,0937+505,1026+453,1033+464,1040+451,1041+580$, $1057+719,1109+244,1125-025,1145+187,1234+481,1254+223,1403-077,1415+132,1636+351$, and $2357+296$. However, 12 EUV-detected stars, believed to be within a PG field as listed in the survey paper, and measured to have $B$ magnitudes within the $m_{b}$ color limit listed for that field, do not appear in PG. (Other EUV detected stars having V magnitudes from Vennes et al. (1997b) fainter than the magnitude limit of the overlapping PG field are ignored.) The 12 include 5 - PG 0232+035 (Feige 24), 1123+189, 1232+238, 1415+132, and 2309+105 which are in the PG, but in fields which for reasons of quality were not retained in the final sample of the catalog. We omit $0845+488 \mathrm{~J}$, as well, since it has a luminous A companion (HD 74389A) that would prevent its discovery in a UV-excess survey. The remaining six undetected stars, named by the J2000 coordinates used in the EUV survey publications, are 1016-053J, 1032+534J, 1043+490J, 1426+500J, 1529+486J, and 1650+406J.

We thus find that 18 of 24 EUV-selected stars in PG fields are in the complete PG sample, or $75 \%$. This falls between the estimates of Darling (1994, 58\% for white dwarfs) and Kilkenny et al. (1997, 84\% for completeness to the stated magnitude limit). The latter value agrees with the assessment in the PG paper itself. Our judgment is to adopt this intermediate value of $75 \%$, given that the Darling (1994) result was probably influenced by the color incompleteness, a problem which the PG project clearly underestimated. At the same time, the evidence from the EUV comparison is that the PG estimate was too optimistic. However, we have to regard this completeness correction as uncertain by at least $15 \%$.

There is one remaining worry about the application of this test: Four of the six "missed" EUV-selected stars have V magnitudes of 13-14. These magnitudes are near the brightness limit of the film. The possibility exists that the photographic $m_{u}$ or $m_{b}$ could be reaching saturation at these brightnesses, so that the color difference was corrupted. This is difficult to quantify, since there is no hard magnitude limit. It is possible that apparently-bright 
stars that have been missed could have added disproportionally more to the $1 / V_{\max }$ density.

\section{THE FORMATION RATE OF WHITE DWARFS}

\subsection{Formation Rate of the DA White Dwarfs in PG}

The more complete picture of the DA white dwarfs is to take the space densities and consider their evolution times as functions of both mass and luminosity. In Figure 16 the number density of white dwarfs weighted by $1 / V_{\max }$ is plotted for 0.5 dex intervals of the log of the cooling time, for the three mass components shown in Figure 14. The evolution rates per unit time interval are listed in Table 3. The local formation rate of white dwarfs is not expected to have varied rapidly over the last Gyr or so. We thus expected the evolution rates for each of the three mass components to be roughly constant, as long as there are enough stars per bin and the samples are complete.

For the $0.6 M_{\odot}$ peak component, the rates are similar for the first four bins through $\log \tau<$ about 7.75. This corresponds roughly to a $T_{\text {eff }}$ near $23,000 \mathrm{~K}$ and $M_{V} \sim 10.5$. For the oldest four bins, however, the rates decline rapidly. This indicates the onset of incompleteness, due in all probability to the increasingly red $m_{u}-m_{b}$ color of the stars. The redder a star is, the more likely it was not found in the PG. The dispersion of photographic $m_{u}-m_{b}$ must be large indeed for any DA stars approaching 25,000 K to be missed. This decline in the rates tracks the progressive decline of the overall LF compared with that of Darling (1994) for the fainter $M_{V}$ bins. A rough weighted mean of the first three bins of Table 3 indicates that the formation rate of dominant $0.6 M_{\odot}$ component DA white dwarfs is thus most likely around $4.5 \pm 0.4 \times 10^{-13} \mathrm{pc}^{-3} \mathrm{yr}^{-1}$. The error estimate is based on the dispersion of the first three bins. A single star with a CO core usually dominates the light, but a fraction of these may be double degenerates, for which no enhancement to the rate is applied. Moreover, no correction has yet been applied for the likely incompleteness due to the PG magnitude limit discussed by Darling (1994) and Kilkenny et al. (1997).

The other two components contribute appreciably to the overall formation rate of white dwarfs. Inspection of Figure 15 reminds us, however, that the onset of incompleteness due to $m_{u}-m_{b}$ color occurs at different $M_{V}$ values. We can make a rough estimate of the contributions of the other two components, but the smaller sample sizes make these even less rigorous.

The low mass $\left(0.4 M_{\odot}\right)$ component reaches a similar color $(25,000 \mathrm{~K})$ at $M_{V}$ near +9

and $\log \tau$ of 7 . Based on $\sim 10$ stars, the rough formation rate is near $0.4 \times 10^{-13}$ in the same units. 
Since we intend to count the formation rate of all white dwarfs, in binaries as well as single stars, our calculated rate requires enhancement because most of the low mass stars are really double. Marsh et al. (1995) found most of the low mass PG stars to be radial velocity variables, though Maxted \& Marsh (1999) investigated two that appeared not to vary. The SPY project is a large, general observational program to find close binaries from several white dwarf surveys, mainly as potential progenitors for SNIa (Napiwotzki et a. 2001; Koester et al. 2001). Detailed statistics are not yet available, but the yield of radial velocity binaries from stars with low mass estimates appears to be high. When the primary star (in apparent magnitude) is a helium white dwarf, the companion can have a core composed of He or CO. Admittedly, Zuckerman \& Becklin (1992) found that a few of the low mass PG white dwarfs harbor low mass nondegenerate companions. These should also be close, probably pre-cataclysmic binaries with only one helium white dwarf.

Our conclusion is that, to account for the degenerate companions to the observed low mass white dwarfs, the overall formation rate should be $0.6-0.7 \times 10^{-13}$ in the same units. Note that this may include some more massive companions with CO cores. Thus the properlyweighted contribution of the low mass stars is more than an order of magnitude higher than indicated by the space density alone, and accounts for $\sim 10 \%$ of all DA white dwarfs.

There is another reason why the discovery of low mass probable binary white dwarfs may be incomplete: they may be so low in mass, their spectral lines are too narrow for them to be readily recognized as white dwarfs. The discoveries by (1) Heber et al. (2003) that HD 188112, a "bright $V=10$ nearby B-type star" (to quote these authors), is in reality a very low mass $\left(0.24 M_{\odot}\right)$ white dwarf with a helium core, and (2) of several more in the early white dwarf samples from the SDSS (Kleinman et al. 2004; Liebert et al. 2004) with masses as low as $0.19 M_{\odot}$, suggests that the low mass white dwarf component is incomplete in its mass range. The lowest mass white dwarf in the present sample is $0.32 M_{\odot}$. No additional correction to the rate for this kind of incompleteness can yet be made, but the ultra-low mass stars can be found efficiently in the SDSS samples.

For the high mass component, the stars remain sufficiently blue $(25,000 \mathrm{~K})$ to about $M_{V}$ near +11 and $\log \tau \sim 8.0$. Again, with $\sim 10$ stars retained that are sufficiently blue, a rough formation rate of $0.9 \times 10^{-13}$ is inferred. This is $20 \%$ of the rate of the peak $0.6 M_{\odot}$ component, a factor of two smaller than the relative contribution from the space density alone. However, the contribution of the subset with $\geq 1 M_{\odot}$ drops to several per cent from the $14 \%$ contribution to the space density. There are not enough stars to determine a separate, accurate formation rate for this very massive subset.

In the next section, the likely contribution of binary evolution to the component of massive white dwarfs will be evaluated. It is inappropriate to increment the formation rate 
for binaries within this component, as binary evolution would generally result in a merger leaving a single star of high mass. Very few double degenerate systems with very high combined masses have been found so far in the SPY project (Karl et al. 2003).

No correction to the contribution of the massive star rate for those in binary systems is appropriate here. Binary evolution may be responsible for the some of the massive stars - presumably by mergers of white dwarfs of more ordinary mass - as argued previously by from their EUV and soft X-ray-selected hot white dwarfs. If the high mass component is thus a mix of these two origins, very few if any will remain binary, and no enhancement to their rate is appropriate.

Proper weighted by the apparent magnitude limit (radius), and by cooling time, the low and high mass components account for $15 \%$ and $20 \%$ of all hot DA white dwarfs.

Thus, our estimate of the total formation rate of hot DA white dwarfs is about $6 \times 10^{-13} \mathrm{pc}^{-3}$ $\mathrm{yr}^{-1}$. Of these $10 \%$ are of low mass (or have at least one binary component of low mass), and a striking $15 \%$ are more massive than $0.8 M_{\odot}$. The total estimate is likely uncertain by at least $10 \%$ based on the scatter in the rate determinations.

\subsection{Inclusion of DB and DO White Dwarfs}

To get the total formation rate for all hot PG white dwarfs, one must also include the 51 DB and 9 DO stars found in complete sample. A similar analysis of these stars has not yet been completed. We do know that the temperature (and age) distributions of the DB-DO and DA sequences differ in that the DB-DO do not include any stars between 30,000 K and 45,000 K (the so-called "DB gap"). The DA sequence overlaps the DB-DO sequence at other temperatures. For now, we can only assume that, on average, the same $V_{\max }$ weighting distribution applies for these stars. This would give us an increment of $60 / 347$ or $17 \%$. If the spectroscopic analysis of a large sample of DB stars by Beauchamp (1996) and Beauchamp et al. (1996) is correct, nearly all of the stars with helium atmospheres are part of the 0.6 $M_{\odot}$ component. It should be noted, however, that the more modest study of DB stars by Koester et al. (2001) found systematically higher masses. Anyhow, the overall formation

rate is lifted to $7 \times 10^{-13} \mathrm{pc}^{-3} \mathrm{yr}^{-1}$. It is impossible to assess how much is the uncertainty in this non-DA rate increment, though it is a relatively minor effect. 


\subsection{Catalog Incompleteness Due to the Magnitude Limit}

Our comparison with lists of EUV detections discussed earlier favors $75 \%$ as the best educated guess as to completeness to the stated magnitude limits of individual PG fields. This applies for only white dwarfs hotter than about 25,000 K for reasons discussed earlier. With this assumption, the overall $\mathrm{PG}$ rate is multiplied by $1 / 0.75$, to give a corrected rate of $0.93 \times 10^{-12} \mathrm{pc}^{-3} \mathrm{yr}^{-1}$. The optimistic assessment of $82 \%$ catalog completeness from Kilkenny et al. (1997) and the original PG paper would yield $0.85 \times 10^{-12} \mathrm{pc}^{-3} \mathrm{yr}^{-1}$, while the pessimistic Darling (1994) estimate (58\% for white dwarfs) would yield 1.2 in the same units. The uncertainty in the completeness estimate is at least $15 \%$.

\subsection{Incompleteness due to White Dwarfs Lost from PG due to Luminous Companions}

A further contribution to the formation rate of white dwarfs comes from undetected white dwarfs in binary systems containing luminous nondegenerate companions. Such systems, typified by Sirius and Sirius B, are often called Sirius-like systems. However, unresolved main sequence companions as cool as K stars may prevent selection of the white dwarf by UV excess. The relative frequency of these systems is highly uncertain since the white dwarf components are effectively obscured at optical wavelengths by the luminosity of the companion. In the past they have mainly come to light through radial velocity variations of the primary or in some cases, were accidentally discovered as wide binary common proper motion pairs. Recently, however, observations in the vacuum UV and the EUV have led to the discovery of many more such systems. At these short wavelengths, even moderately hot white dwarfs $\left(M_{V}<12\right)$ can dominate the composite energy distribution.

A survey of existing literature (by JBH) indicates that there are approximately 41 Siriuslike systems with white dwarf companion of types B through $\mathrm{K}$, most having been discovered at EUV wavelengths. A relatively firm lower limit to the relative number of such systems can be obtained from the known white dwarfs within 20 pc of the Sun (Holberg, Oswalt \& Sion 2002). Of the 109 white dwarfs in this sample, $25 \%$ are in binary systems, mainly with M dwarfs. All four Sirius-like systems in the sample lie within $14 \mathrm{pc}$ of the sun. This can be taken as a reasonable lower limit of $\sim 4 \%$ on the relative frequency of such systems.

The actual number is expected to be significantly higher, however. Some evidence that this is indeed the case comes from the FAUST UV camera flown on the space shuttle Atlas-1 mission (Bowyer et al. 1993). Detailed studies of 14 selected FAUST fields (Fromiggini et al. 2003) detected some 14 main sequence stars with strong evidence of UV excesses, where 
only 3 to 5 would have been expected based on the number of EUV detections. The EUVE and ROSAT EUV surveys doubtlessly failed to detect many Sirius-like white dwarfs, due to (1) the distance limitations imposed by the absorption in the interstellar medium, (2) effective temperature biases due to a lower limit of $T_{\text {eff }}=25,000 \mathrm{~K}$ for detecting any white dwarf, and (3) the rapid increase of EUV opacity in most DA stars above $T_{\text {eff }}=50,000 \mathrm{~K}$. Finally, consideration of the cumulative $\log \mathrm{N}-\log$ distance distribution for 39 Sirius-like systems, having firm distance estimates, gives a slope of only +1 , where a slope of +3 slope is expected for a reasonably complete sample.

In summary, an uncertain increment of around $10 \%$ to account for white dwarfs hidden by luminous companions appears appropriate. We shall include this tacitly in the final estimate. A program has been initiated using the GALEX (Galaxy Evolution Explorer) mission to produce hopefully a much better estimate of the fraction of hot white dwarfs hidden by companions.

\subsection{The Total White Dwarf Formation Rate}

In conclusion the total, corrected rate of recent white dwarf formation appears to be close to $1 \times 10^{-12} \mathrm{pc}^{-3} \mathrm{yr}^{-1}$. Our best educated guess is that this is uncertain by \pm 0.25 in the above units, but this is obviously poorly constrained.

It may be worthwhile to compare this rate applicable to white dwarf formation in the last $10^{8}$ years or so to estimates of the average formation rate over the lifetime of the Galactic disk. A rough estimate may be easily obtained. The local density of all white dwarfs is arguably complete to a distance of about $13 \mathrm{pc}$, based on the cumulative distribution function (Holberg, Oswalt \& Sion 2002), and is $5.0 \pm 0.7 \times 10^{-3} \mathrm{pc}^{3}$. Since these and other cool white dwarfs fit cooling ages no older than 8 Gyr (BLR), the average white dwarf formation rate averaged over the lifetime of the disk is about $6 \times 10^{-13} \mathrm{pc}^{-3} \mathrm{yr}^{-1}$. It is not surprising that the current formation rate is nearly twice as high, since new white dwarfs are drawn from progenitors of decreasing average mass.

\subsection{Other Estimates of the WD Formation Rate}

Vennes et al. (1997b) estimated a DA white dwarf formation rate of $0.7-1.0 \times 10^{-12} \mathrm{pc}^{-3}$

$\mathrm{yr}^{-1}$ based on a sample of over 100 hot white dwarfs detected in the EUVE. Objects fall between 25-75,000 $\mathrm{K}$ and most have $\tau<30 \mathrm{Myr}$. It may be argued that EUV selection may be hampered by neutral hydrogen absorption in the local ISM or heavy element absorption 
in the white dwarf photosphere (Finley et al. 1997; Marsh et al. 1997). Both effects might exclude some white dwarfs from detection, and bias an EUV sample toward higher masses where gravitational diffusion could more likely be effective. However, Vennes et al. (1997b) found that the estimated density based on samples of objects closer than 80-100 pc is only marginally dependent on Galactic coordinates. A cumulative distribution vs. distance of 110 DA stars is fairly well reproduced by a $d^{3}$ slope up to 80-90 pc.

Eight stars from this PG sample have $T_{\text {eff }}>25,000 \mathrm{~K}$ and estimated distances $<90 \mathrm{pc}$. Of these, six are detected by the EUVE. Moreover, the Vennes et al. (1997b) formation rate is in fairly good agreement with that reported here after correction of the PG DA sample for catalog incompleteness and luminous binary companions.

The older Weidemann (1991) estimate of $2.3 \times 10^{-12}$ has often been cited by those comparing the formation rate of planetary nebulae $(\mathrm{PN})$. However, this result presented in a conference proceeding is not based on a study of a hot white dwarf sample. Rather, it is based on the star, white dwarf formation model over the lifetime of the Galactic disk by Yuan (1992), mentioned earlier. Moreover, the rate would be only $60 \%$ of this value if a $67 \%$ correction for incompleteness due to luminous companions were not included. The rate does agree with recent estimate of the $\mathrm{PN}$ formation rate, as we will discuss. If our binary correction of $10 \%$ were applied, the Weidemann estimate is actually in fair agreement with ours and Vennes et al. (1997b) estimates.

Darling (1994) did not calculate an overall formation rate of DA white dwarfs. It may be possible to correct the $M_{V}$ bias and make such an estimate. It would likely exceed our uncorrected $\mathrm{PG}$ rate, but not necessarily the corrected rate.

\subsection{Comparison with PN Formation Rate}

To determine an accurate formation rate of PN in the Galaxy has been a notoriously difficult problem. Pottasch (1996) notes that published values differ from $0.4-8 \times 10^{-12} \mathrm{pc}^{-3}$ $\mathrm{yr}^{-1}$. There are two main reasons why this rate is difficult to determine:

(1) Distance estimates for Galactic PN are obtained by a variety of methods, but traditional distance scales vary over a factor of 2 , which translates to a factor of 8 in space density.

(2) Lifetimes of the PN phase are very uncertain. For example, in individual cases, there is often disagreement by an order of magnitude between a nebular kinematic age (several $\times 10^{4}$ ) and the evolutionary age of the central star (several $\times 10^{5}$ ) based on its mass and 
position in the HR Diagram.

The best estimates of the space density of PN utilize a local sample, where the distances may be estimated by more robust techniques, such as a trigonometric parallax. However, this still requires an accurate census of sources close to the Sun, as well as distances and post-AGB ages. Two such recent studies may be the most reliable attempts for Galactic PN.

For a restricted cylinder of radius $1 \mathrm{kpc}$ and vertical length $0.64 \mathrm{kpc}$, Phillips (2002) gets $2.1 \times 10^{-12} \mathrm{pc}^{-3} \mathrm{yr}^{-1}$. A difficulty with the comparison to white dwarfs, even with a "local" sample, is that the use of a much larger sample size for PNs like the above is inevitable, and leads to a considerable dependence on the assumed vertical $(z)$ scale height. Pottasch (1996) gave an estimate of the local PN formation rate based on distances to 50 nearby PN determined individually by a variety of methods. Using a $0.7 \mathrm{kpc}$ cylinder around the sun, his value is $3 \times 10^{-12} \mathrm{pc}^{-3} \mathrm{yr}^{-1}$.

Many researchers might conclude that estimates of the formation rate of WD and PN differing by only a factor of 1.8 to 2.6 (for each of the above estimates) are not in serious disagreement, given the many uncertainties. On the other hand, the PN are only a subset of the objects becoming WD. The PN phase probably must have been preceded by a full AGB phase. Stars which for any reason have lost much of their hydrogen envelopes in their earlier red giant evolution may evolve off the AGB at a lower luminosity and core mass. These may have post-AGB evolutionary times too slow to ionize the ejected PN gas before it is dispersed. There are more "UV-bright" post-AGB stars observed in globular clusters than there are PN. Hence, WDs emerging from the oldest and lowest mass progenitors as single stars may not produce PN. Some horizontal branch stars have lost so much hydrogen envelope that they do not enter the AGB phase at all, and almost certainly do not produce PN. Heber (1986) has estimated that about $2 \%$ of WD progenitors go through only such a subdwarf B phase of core-helium burning. While the majority of WD progenitors in the disk population probably do pass through a full AGB phase, it is not clear how close to $100 \%$ that fraction is. Especially intriguing is the growing evidence that a large fraction of $\mathrm{PN}$ central stars are binary (De Marco et al. 2004), suggesting to some that single stars might not produce $\mathrm{PN}$ at all!

In summary, it is not clear how serious is the lingering disagreement between our estimate of recent WD formation rate and the recent, probably best estimates of the PN rate. 


\section{ORIGIN OF THE MASSIVE WHITE DWARF COMPONENT}

The $15 \%$ contribution of stars more massive than $0.8 M_{\odot}$ to the rate of formation of all DA white dwarfs would seem surprisingly high, based on most previous studies. We have already pointed out the suggestions by Marsh et al. (1997) and Vennes (1999) - and even earlier by Schmidt et al. (1992) and BSL - that binary mergers may account for a substantial fraction of these stars. To our knowledge, the hypothesis of a binary contribution has never been evaluated quantitatively.

To assess this possibility, one must first know the expected contribution to the overall DA white dwarf formation rate from single, presumably-massive progenitors. In general, single white dwarfs of $<0.8 M_{\odot}$ are expected to evolve from $\geq 4 M_{\odot}$ progenitors, which are rare compared to less massive stars. The only theoretical calculation of the expected mass distribution from single stars we are aware of is the dissertation work of Yuan (1992) - see also Weidemann \& Yuan (1989). Here the overall white dwarf formation rate, mass and luminosity distributions over the lifetime of the Galactic disk were calculated, based on a stellar initial mass function (IMF), a star formation rate, and an initial/final mass relation. A graphical display of one version of the predicted mass function is shown in Figure 11 of Yuan (1992). This particular choice assumes (1) a constant star formation rate, (2) a Scalo IMF, (3) white dwarf evolution times from Wood (1990), (4) a 12 Gyr disk age, and (5) the initial/final mass relation of Weidemann (1987). These choices still appear reasonable today, but also the shape of the derived mass distribution appears from the plots in the paper to be pretty insensitive to reasonable variations of these five choices.

We have performed a graphical measurement of areas under the "unfolded" mass distribution curve in this figure. "Unfolded" means no allowance was made for dispersion in the assumed monotonic initial/final mass relation, an irrelevant consideration here. The curve spanning $0.5-1.2 M_{\odot}$ was divided into ten arbitrarily chosen mass intervals and linear segments were drawn across each. The mass intervals $0.5-0.55,0.55-0.575,0.575-0.60$, $0.60-0.65,0.65-0.7,0.7-0.75,0.75-0.8,0.8-0.9,0.9-1.0$, and $1.0-1.2 M_{\odot}$. Finer intervals were chosen where the curve was changing slope the most. Areas were measured under each chord to assess its contribution to the overall predicted mass. For the components $<$ and $>0.8 M_{\odot}$, respectively, the areas (arbitrary units) are 172 and 6 units. For the $>0.8 M_{\odot}$ component, the subset $>1.0 M_{\odot}$ is 2 units.

The above exercise indicates that the Yuan (1992) calculation predicts that the $>0.8$ $M_{\odot}$ component forms only $3.5 \%$ as many stars as the $<0.8 M_{\odot}$ "peak" component. The low mass component produced from binary evolution is not part of this calculation. This compares with $20 \%$ as many high mass stars as "peak" stars from the rates of the previous section. The Yuan (1992) results are given in units of $\mathrm{pc}^{-3}$, and hence allow for the magnitude 
limit correction. The use of cooling models from Wood (1990) indicates that evolution times have also been allowed for. The only difference here is that the calculated mass distribution includes white dwarfs with luminosities down to below $10^{-4} L_{\odot}$.

The result of the calculation is that this model for white dwarf formation from single star evolution predicts only $17.5 \%$ of the observed rate of formation of the massive white dwarfs. Binary evolution is required for 82.5\%! We argue in Appendix A, however, that a larger fraction of the very hot, EUV-selected white dwarfs might come from single-star evolution due to the associations of massive stars distributed around the Sun nearer the Galactic plane.

\section{COMMENTS ON INDIVIDUAL OBJECTS}

\subsection{Magnetic White Dwarfs}

This sample includes 10 white dwarfs with detected magnetic fields. Eight of these have an estimate of the mass, from methods discussed in Liebert et al. (2003). These authors use this and other samples to document an increasingly-strong case that the average mass of magnetic white dwarfs is substantially higher than peak mass. The mean mass of the eight PG stars is $0.93 M_{\odot}$ - including PG $1658+441$ at $1.31 M_{\odot}$ (Schmidt et al. 1992), the highest determination in the sample. The radius bias discussed in $\S 3$ leads to the conclusion in the cited paper that the true frequency of magnetic white dwarfs with fields exceeding about 2 megagauss may exceed $10 \%$ of all white dwarfs. This is before allowing for more difficult-to-detect fields less than this value.

\subsection{Peculiar DAB and Composite Objects}

Several stars with basically hydrogen-rich atmospheres but showing helium lines are found in the survey, usually with temperatures in the 30,000-45,000 K range of the so-called "DB gap" (Liebert et al. 1986). GD 323 (PG 1302+597) is a peculiar DAB star at about $30,000 \mathrm{~K}$ whose spectrum could be fit with neither a homogeneous, mixed H/He model. A more promising, but unproven possibility is that its $\mathrm{H}$ and He layers are spatially stratified in some manner (Liebert et al. 1984). Since the parameter determinations for this star are not accurate, we have little choice but to omit it from the complete PG sample.

PG 1305-017 and PG 1210+533 are peculiar DAO stars Bergeron et al. (1994). The

former is best fit with a stratified model at $T_{\text {eff }}$ near $44,000 \mathrm{~K}$. The latter $(\sim 45,000 \mathrm{~K})$ shows 
variability in the strengths of the He I and He II lines, perhaps due to spatial variation in the $\mathrm{H} / \mathrm{He}$ ratio. Most DAO stars are best fit with homogeneous atmospheres of mixed $\mathrm{H}, \mathrm{He}$ composition, and are not known to be spectrum variables (Bergeron et al. 1994).

The hydrogen-rich PG 1603+432 near 37,000 K was recently shown by Vennes, Dupuis, \& Chayer (2004) to have a He II $1085 \AA$ line in spectrum obtained with the Far Ultraviolet Spectroscopic Explorer. These authors predicted that He I $4471 \AA$ should be detected in a spectrum of high signal-to-noise ratio. These authors pointed out that the line may have been marginally detected in a spectrum published by Bergeron et al. (1994). Indeed, we have obtained a second, better spectrum, and the He I line indeed seems to be there. Both the old and new spectra of this star are shown in Figure 17. However, the best fit to the far-UV spectrum that Vennes, Dupuis, \& Chayer (2004) obtained was with a homogeneous atmosphere with $\mathrm{He} / \mathrm{H}=0.01$. This predicts a He I $4471 \AA$ line that is distinctly stronger than observed in both of these spectra. This star may thus be a spectrum variable, or require a more complicated H,He layering.

The DAB star PG $1115+166$, as mentioned in $\S 2.3$, requires a quite different explanation. Bergeron \& Liebert (2002) showed that the spectrum is best explained as a double degenerate where one component is a DA star with $T_{\text {eff }}$ of $22,000 \mathrm{~K}$, accompanied by a DB star near $T_{\text {eff }}$ of $16,210 \mathrm{~K}$. In a similar time frame, Maxted et al. (2002) found this to be a 30.09-day period double degenerate system.

\subsection{ZZ Ceti Stars}

The PG sample extends to low enough effective temperatures to cover the range where ZZ Ceti pulsators are found, between about $T_{\text {eff }}=12,500 \mathrm{~K}$ and $11,100 \mathrm{~K}$ according to the detailed study of Bergeron et al. (1995). At the beginning of our project, the PG sample included 9 previously known ZZ Ceti stars, all of which were found within the empirical instability strip. As part of our survey, PG 1349+552 (LP 133-144) was also found to lie within the strip, and further high-speed photometric observations by Bergeron et al. (2004) confirmed that it was indeed a new variable white dwarf. Our results for the PG survey are thus consistent with the conclusions of Bergeron et al. (2004, and references therein) that the empirical instability strip contains no non-variable stars, in sharp contrast with the results of Mukadam et al. (2004) who claim, based on the analysis of the DA stars in the Sloan Digital Sky Survey, that the ZZ Ceti instability strip contains a significant fraction of non-variable stars. 


\subsection{Two Unusual, Very Hot, Hydrogen-rich White Dwarfs}

We could not end a section on the "unusual" objects in PG without mention of what may be the two hottest DA stars in the sample:

BE UMa (PG1155+492) is the only pre-cataclymic binary we are aware of in the sample. It consists of a DAO or high-gravity sdO paired with a $\mathrm{K}$ star at a 2.2-hour period (Ferguson et al. 1999) - and references therein. The reprocessing spectrum due to radiation intercepted by the facing side of the secondary is one of the most impressive known. Of the known sample of such binaries, this is one of the most recent to have emerged from the common envelope evolution phase, which may account for why the $\mathrm{K}$ star is oversized compared with main sequence stars of similar temperature. The $T_{\text {eff }}$ and $\log g$ values are not considered accurate enough in include it in the PG analysis here.

The planetary nebulae EGB $6=$ PG 0950+139 is a unique, unsolved problem of stellar astrophysics. The central star has a poor $T_{\text {eff }}$ estimate of $108,000 \mathrm{~K}$, though it is still included in Table 1 and our analysis. There is an old, low-surface-brightness PN shell (Ellis, Grayson, \& Bond 1984), not unexpected for a central star with these parameters. What is unique about the object, however, is that there is a very compact component to the PN (Liebert et al. 1989), very uncharacteristic of a central star with these parameters. Several arguments constrain the dimensions of this extremely-dense, nebular component to the order of 10 a.u. An unusual hypothesis to explain the object - the ablation of a Jovian planet by its very hot star (Dopita \& Liebert 1989) - is apparently ruled out by imaging and spectra taken with the Hubble Space Telescope (Bond et al. 1993). The mystery remains.

\section{SUMMARY AND FUTURE ENDEAVORS}

While a greater appreciation of the incompleteness of the PG Survey - particularly at lower temperatures - over that envisioned earlier has hampered our efforts, we believe that this thorough study of the sample using homogeneous observations and improved theory has led to some interesting, even unexpected conclusions. When the sample is normalized to the same volume, there are nearly two thirds as many high mass DA stars $(39.1 \%$ of the total) with $\geq 0.8 M_{\odot}$, as are in the peak $\left(0.46-0.8 M_{\odot}\right)$ component $(60.1 \%)$. Yet, the low mass component - so prominent in the plot of the unweighted mass distribution - accounts for only $0.8 \%$ of the space density of hot DA stars.

While the accounting for white dwarf radii has a big impact on a apparent-magnitude limited sample, so does consideration of the evolutionary times also as a function of mass. Thus, the peak, low and high mass components contribute $75 \%, 10 \%$, and $15 \%$ to the 
overall formation rate of DA white dwarfs, respectively. The calculation discussed in $\S 6$ appears to require that $\geq 80 \%$ of the massive white dwarfs come from binary star evolution, presumably mergers of two white dwarfs of smaller mass. To our knowledge, neither the observed formation rates as a function of mass components, nor the relative contribution of binaries to the high mass component, have been rigorously evaluated previously.

When the overall formation rate is corrected for incompleteness of the catalog (as best as we are able to assess it), for DB-DO stars, for those hidden by luminous non-degenerate companions, and for those white dwarfs which are likely to be double degenerates, we estimate a total, recent formation rate of white dwarfs in the local Galactic disk of $1 \pm 0.25 \times 10^{-12} \mathrm{pc}^{-3}$ $\mathrm{yr}^{-1}$. It is difficult to know if this error estimate encompasses all possible systematic errors. Agreement with previous determinations for white dwarfs is good. The best estimates of the formation rate of planetary nebulae still appear to be around twice as high. Although less of a discrepancy than appeared to be the case at the time of the publication of Fleming et al. (1986), this may still be somewhat worrisome, since not all white dwarfs pass through a planetary nebula phase. A rigorous, similar analysis for DB stars is also needed.

In the near future, larger samples with better completeness assessment and homogeneous observations can be brought to bear on this problem. The SPY project, though its primary purpose is to search for double degenerate systems using radial velocities, should produce robust determinations of the white dwarf parameters for 1500 stars Koester et al. (2001). Data from the Sloan Digital Sky Survey has already been mined to produce a catalog of over 2,500 mostly-new, mostly-hot white dwarfs Kleinman et al. (2004). These come with photometric observations in five $(u, g, r, i, z)$ bands, and with blue and red spectrophotometry. A limitation is that the short-wavelength cutoff of the spectra near $\lambda=3830 \AA$ excludes the highest Balmer lines for the bulk of DA white dwarfs, and this compromises primarily the measurement of the gravity. In any case, it is hoped that this analysis of the PG sample will serve as a suitable "benchmark" against which the expected improvements from such samples as these can be measured.

The PG sample will retain one advantage over those from the larger, deeper surveys: this is a sample which contains many of the brightest, hot white dwarfs known. The availability of accurate parameters should support followup observations with FUSE and other future space missions. Earlier it was mentioned that GALEX may produce a more rigorous determination of the fraction of hot white dwarfs hidden by companions.

We acknowledge S. Boudreault, A. Gianninas, R. A. Saffer, and D. K. Sing for the acquisition and data reduction of spectra used in this analysis. We thank I. Hubeny for calculating LTE and NLTE model atmospheres for comparison with our calculations. JL 
acknowledges useful conversations with Richard Green concerning the completeness of the PG, Lars Bildsten concerning the strategy of this analysis, and Eric Mamajek concerning the Gould's Belt population. We thank the referee and Professor Volker Weidemann for careful readings of this paper, and for helpful criticisms, suggestions and comments. This work was supported in part by the National Science Foundation through grant AST-0307321, by the NSERC Canada, by the Fund FQRNT (Québec), and by NASA grant NAG5-9408. 


\section{A. MASSIVE, EUV-SELECTED WHITE DWARFS AND GOULD'S BELT?}

We have shown in this paper that, for the PG high Galactic latitude sample, $\geq 80 \%$ of the DAs with $>0.8 M_{\odot}$ may require a formation mechanism from binary evolution, rather than from massive (4-8 $M_{\odot}$ ) single progenitors. It is interesting to examine briefly the white dwarf counterparts found in the EUVE and ROSAT all-sky surveys, whose parameters are published in Vennes et al. (1996), Marsh et al. (1997), Vennes et al. (1997b), and Vennes (1999). There are 28 white dwarfs from these sources with estimated masses $>0.8 M_{\odot}$, in fact 19 have $>1 M_{\odot}$. Note that this is a much higher fraction with $>1 M_{\odot}$ than is the case for the PG sample. All are hotter than 25,000 K, and all but three are above 30,000 K. The latter temperature corresponds to a cooling age near $10^{8}$ years for a $1 M_{\odot}$ white dwarf. Thus most of these stars have total ages of a few $\times 10^{8}$ years or less.

These white dwarfs are plotted (with "star" symbols) vs. Galactic coordinates in Figure 18. While these stars were detected in an all-sky survey, it is interesting that all but nine are within \pm 30 degrees Galactic latitude. The overlap with PG (whose stars are all above this latitude) is small. Most of the massive PG white dwarfs are too cool to be EUV sources.

The Sun appears to reside currently in a region of enhanced, recent star formation in the Galactic disk. Historically, Gould (1874) noted that the brightest stars in the sky lie in a "belt" tilted some $\sim 18^{\circ}$ from the Galactic plane reaching farthest south near $180^{\circ}$ Galactic longitude. This distribution, dominated by B and O stars, is referred to as Gould's Belt. In Figure 18 we have added as small dots all the B and $\mathrm{O}$ stars from the on-line version of the Yale Bright Star Catalog (Hoffleit 1991). An analysis of an earlier version of this sample is presented in Bahcall et al. (1987). The agreement in positions of the massive EUV white dwarfs and the O,B stars suggests an association. The distribution of white dwarfs admittedly also depends on the very nonuniform interstellar EUV opacity for distances out to a few hundred parsecs.

A comprehensive analysis of Hipparcos astrometry by De Zeeuw et al. (1999) identified $18 \mathrm{OB}$ associations within this range of Galactic latitude, located at distances ranging from 100 to roughly $600 \mathrm{pc}$. The youngest of these entities such as the Sco OB2 complex are probably too young (a few Myr to perhaps $20 \mathrm{Myr}$ ) to have produced any white dwarfs. However, the older (of order $100 \mathrm{Myr}$ ) of these associations at 100-200 pc distances, such as the $\alpha$ Per and Cas-Tau associations, may have provided suitable progenitors for massive white dwarfs. We thus suggest that a significantly higher fraction of the massive EUV white dwarfs near the Galactic plane could have evolved from single-star progenitors than for those at high Galactic latitudes. Finally, we surmise that the Gould Belt associations may not generally be old enough for white dwarfs in the $0.8-1.0 M_{\odot}$ range to have yet been produced. 


\section{REFERENCES}

Althaus, L. G., Serenelli, A. M., \& Benvenuto, O. G. 2001, MNRAS, 323, 471

Bahcall, J., Casertano, S., \& Ratnatunga, K. U. 1987, ApJ, 320, 515

Barstow, M. A., Good, S. A., Burleigh, M. R., Hubeny, I., Holberg, J. B., and Levan, A. J. 2003, MNRAS, 344, 582

Barstow, M. A., Good, S. A., Holberg, J. B., Hubeny, I., Bannister, N. P., Bruhweiler, F. C., Burleigh, M. R., \& Napiwotzki, R. 2003, MNRAS, 344, 870

Beauchamp, A. 1996, Ph. D. dissertation, Université de Montréal

Beauchamp, A., Wesemael, F., Fontaine, G., Lamontagne, R., Saffer, R. A., \& Liebert, J. 1996, in Hydrogen-Deficient Stars and Related Objects, ASP Conf. Proc. vol. 96, eds. C.S. Jeffery \& U. Heber (San Francisco CA: Astronomical Society of the Pacific), 295

Bergeron, P. 1993, in White Dwarfs: Advances in Observation and Theory, NATO ASI Series, ed. M. A. Barstow (Dordrecht: Kluwer Academic Publishers), 267

Bergeron, P., Fontaine, G., Billères, M., Boudreault, S., \& Green, E.M. 2004, ApJ, 600, 404

Bergeron, P., Leggett, S. K., \& Ruiz, M. T. 2001, ApJS, 133, 413

Bergeron, P., \& Liebert, J. 2002, ApJ, 566, 1091

Bergeron, P., Saffer, R. A., \& Liebert, J. 1992, ApJ, 394, 228 (BSL)

Bergeron, P., Wesemael, F., Beauchamp, A., Wood, M. A., Lamontagne, R., Fontaine, G., \& Liebert, J. 1994, ApJ, 432, 305

Bergeron, P., Wesemael, F., \& Fontaine, G. 1991, ApJ, 367, 253

Bergeron, P., Wesemael, F., Fontaine, G., \& Liebert, J. 1990, ApJ, 351, L21

Bergeron, P., Wesemael, F., Lamontagne, R. \& Chayer, P. 1993, ApJ, 407, L85

Bergeron, P., Wesemael, F., Lamontagne, R., Fontaine, G., Saffer, R. A., \& Allard, N. F. 1995, ApJ, 449, 258

Bond, H. E., Meakes, M. G., Liebert, J. W., \& Renzini, A. 1993, in Planetary Nebulae, Proc. IAU Symp. 155, eds. R. Weinberger \& A. Acker (Dordrecht: Kluwer Academic Publ.), 499 
Bowyer, S., Sasseen, T. P., Lampton, M., \& Wu, X. 1993, ApJ, 415, 875

Bragaglia, A., Renzini, A., \& Bergeron, P. 1995, ApJ, 443, 735

Darling, G. W. PhD Thesis, Dartmouth College, Hanover NH

De Marco, O., Bond, H. E., Harmer, D., \& Fleming, A. J. 2004, ApJ, 602, L93

De Zeeuw, P. T., Hoogerwerf, R., de Bruijne, J. H. J., Brown, A. G. A., \& Blaauw, A. 1999, AJ, 117,354

Dopita, M. A. \& Liebert, J. 1989 ApJ, 347, 910

Dreizler, S., \& Werner, K. 1996, A\&A, 314, 217

Ellis, G. L., Grayson, E. T., \& Bond, H. E. 1984, PASP, 96, 283

Ferguson, D. H., Liebert, J., Haas, S., Napiwotzki, R. \& James, T. A. 1999, ApJ, 518, 866

Finley, D. S., Koester D., \& Basri, G. 1997, ApJ, 488, 375

Fleming, T. A., Liebert, J., \& Green, R. F. 1986, ApJ, 308, 176

Fromiggini, L., Brosch, N., Almozino, E., Bowyer, S., \& Lampton, M. 2003, MNRAS, 332, 441

Goldschmidt, P., Miller, L., La Franca, F., \& Cristiani, S. 1992, MNRAS, 256, 65p

Gould, B. A. 1874, Proc. AAAS, 115

Green, R. F. 1980, ApJ, 238, 685

Green, R. F., Schmidt, M., \& Liebert, J. 1986, ApJS, 61, 305

Greenstein, J. L. 1976, AJ, 81, 323

Heber, U. 1986, A\&A, 155, 33

Heber, U., Edelmann, H., Lisker, T., \& Napiwotzki 2003, A\&A, 411 L477

Hoffleit, D., 1991, it The Bright Star Catalog, (5th ed.; New Haven: Yale University Observatory)

Holberg, J. E., Barstow, M. A., \& Sion, E. M. 1998, ApJS, 119, 207

Holberg, J. B., Oswalt, T. D., \& Sion, E. M. 2002, ApJ, 571, 512 
Homeier, D., Koester, D., Hagen, H.-J., Jordan, S., Heber, U., Engels, D., Reimers, D., \& Dreizler, S. 1998, A\&A, 338, 563

Hubeny, I., \& Lanz, T. 1995, ApJ, 439, 875

Hummer, D. G., \& Mihalas, D. 1988, ApJ, 331, 794

Iben, Jr., I. \& Renzini, A. 1984, Phys. Reports, 105

Karl, C. A., Napiwotzki, R., Nelemans, G., Christlieb, N., Koester, D., Heber, U., \& Reimers, D. 2003, A\&A, 410, 663

Kilkenny, D., O’Donoghue, D., Koen, C., Stobie, R. S., \& Chen, A. 1997, MNRAS, 287, 867

Kleinman, S.G., et al. 2004, ApJ, 607, 426

Koester, D., Napiwotzki, R., Christlieb, N., Drechsel, H., Hagen, H.-J., Heber, U., Homeier, D., Karl, C., Leibundgut, B., Moehler, S., Nelemans, G., Pauli, E.-M., Reimers, D., Renzini, A., \& Yungelson, L. 2001, A\&A, 378, 556

Koester, D., Schulz, H. \& Weidemann, V. 1979, A\&A, 76, 262

Kondo, M., Noguchi, T., \& Maehara, H. 1984, Ann. Tokyo Astron. Obs., 20, 130

Lemke, M. 1997, A\&AS, 122, 285

Liebert, J., \& Bergeron, P. 1995, in White Dwarfs, eds. D. Koester \& K. Werner (Heidelberg: Springer-Verlag), 12

Liebert, J., Bergeron, P., Eisenstein, D., Harris, H. C., Kleinman, S. J., Nitta, A., \& Krzesinski, J. 2004, ApJ, 606, 147

Liebert, J., Bergeron, P., \& Holberg, J. B. 2003, AJ, 125, 348

Liebert, J., Bergeron, P., Schmidt, G. D., \& Saffer, R. A. 1993, ApJ, 418, 426

Liebert, J., Green, R. F., Bond, H. E., Holberg, J. B., Wesemael, F., Fleming, T. A., \& Kidder, K. 1989, ApJ, 346, 251

Liebert, J., Schmidt, G. D., Sion, E. M., Starrfield, S. G., Green, R. F., \& Boroson, T. A. 1985, PASP, 97, 158

Liebert, J., Wesemael, F., Hansen, C. J., Fontaine, G., Shipman, H. L., Sion, E. M., Winget, D. E., \& Green, R. F. 1986, ApJ, 309, 241 
Liebert, J., Wesemael, F., Sion, E. M., \& Wegner, G. 1984, ApJ, 277, 692

Marsh, M. C., Barstow, M. A., Buckley, D. A., Burleigh, M. R., Holberg, J. B., O’Donoghue, D., Penny, A. J., \& Sansom, A. E. 1997, MNRAS, 287, 705

Marsh, T. R., Dhillon, V. S., \& Duck, S. R. 1995, MNRAS, 275, 828

Maxted, P. F. L., Burleigh, M. R., Marsh, T. R., \& Bannister, N. P. 2002, MNRAS, 334, 833

Maxted, P., \& Marsh, T. R. 1998, MNRAS, 296, L34

McCook, G. P., \& Sion, E. M. 1999, ApJS, 121, 1

Mukadam, A. S., Winget, D. E., von Hippel, T., Kepler, S. O., \& Costa, A. F. M. 2004, ApJ, in press

Napiwotzki, R. 1992, in The Atmospheres of Early Type Stars, Lecture Notes in Physics, v. 401, eds. U. Heber \& C.S. Jeffery (Springer: Berlin), 310

Napiwotzki, R. 1997, A\&A, 322, 256

Napiwotzki, R. et al. 2001, Astronomishe Nachrichten, 322, 411

Napiwotzki, R., Green, P. J., \& Saffer, R. A. 1999, ApJ, 517, 399

Noguchi, T., Maehara, H., \& Kondo, M. 1980, Ann. Tokyo Astron. Obs., 18, 55

Oke, J. B. 1969, PASP, 81, 11

Phillips, J. P. 2002, ApJS, 139, 199

Pottasch, S. R. 1996, A\&A, 307, 561

Press, W. H., Flannery, B. P., Teukolsky, S. A., \& Vetterling, W. T. 1986, Numerical Recipes (Cambridge: Cambridge University Press)

Saffer, R. A., Bergeron, P., Koester, D., \& Liebert, J. 1994, ApJ, 432, 351

Saffer, R. A., Keenan, F. P., Hambly, N. C., Dufton, P. L., Liebert, J. 1997, ApJ, 491, 172

Saffer, R. A., Liebert, J., \& Olszewski, E. M. 1988, ApJ, 334, 947

Schmidt, G. D., Bergeron, P., Liebert, J., \& Saffer, R. A. 1992, ApJ, 394, 603 
Schmidt, G. D., West, S. C., Liebert, J., Green, R. F., \& Stockman, H. S. 1986, ApJ, 309, 218

Shipman, H.L. 1972, ApJ, 177, 723

Smith, J. A. et al. 2002, AJ, 123, 2121

Thejll, P., Bauer, F., Saffer, R., Liebert, J., Kunze, D., Shipman, H. L. 1994, ApJ, 433, 819

Vennes, S. 1992, ApJ, 390, 590

Vennes, S. 1999, ApJ, 525, 995

Vennes, S., Dupuis, J., \& Chayer, P. 2004, ApJ, 611, 1091

Vennes, S., Korpela, E., \& Bowyer, S. 1997a, AJ, 114, 1567

Vennes, S., Thejll, P. A., Genova Galvan, R., \& Dupuis, J. 1997b, ApJ, 480, 714

Vennes, S., Thejll, P. A., Wickramasinghe, D. T., \& Bessell, M. S. 1996, ApJ, 467, 782

Wegner, G., \& Darling, G. W. 1994, in Stellar and Circumstellar Astrophysics, eds. G. Wallerstein \& A. Noriega-Crespo, ASP Conf. Series No. 57 (San Francisco CA: Astronomical Society of the Pacific), 178

Wegner, G., \& Schulz, H. 1981, A\&A, 43, 473

Weidemann, V. 1987, A\&A, 188, 74

Weidemann, V. 1991, in White Dwarfs, ed. G. Vauclair \& E.M. Sion (Dordrecht: Kluwer), 230

Weidemann, V. 2000, A\&A, 363, 647

Weidemann, V., \& Yuan, J. W. 1989, in White Dwarfs, Proc. of IAU Coll. 114, Lecture Notes in Physics, ed. G. Wegner (Berlin: Springer-Verlag), 1

Werner, K. 1996, ApJ, 457, L39

Wesemael, F., Auer, L. H., Van Horn, H. M., \& Savedoff, M. P. 1980, ApJS, 43, 159

Wood, M. A. 1990, Ph. D. thesis, Univ. of Texas at Austin

Wood, M. A. 1995, in 9th European Workshop on White Dwarfs, NATO ASI Series, ed. D. Koester \& K. Werner (Berlin: Springer), 41 
Yuan, J. W. 1992, A\&A, 261, 105

Zuckerman, B., \& Becklin, E. E. 1992, ApJ, 386, 260 
Table 1. Atmospheric Parameters of DA Stars from the Complete PG Sample

\begin{tabular}{|c|c|c|c|c|c|c|c|c|c|c|c|c|}
\hline $0000+172$ & & 20,210 & ( 311$)$ & $7.99(0.05)$ & $0.62(0.03)$ & 10.70 & -1.58 & 15.84 & 106 & $5.77(-7)$ & 7.93 & \\
\hline $0004+061$ & PHL 670 & 15,120 & (332) & $7.84(0.05)$ & $0.52(0.03)$ & 10.98 & -2.00 & 16.23 & 112 & $9.60(-7)$ & 8.26 & 1 \\
\hline $0010+281$ & & 26,280 & ( 393$)$ & $7.85(0.05)$ & $0.56(0.02)$ & 9.99 & -1.03 & 15.70 & 139 & $2.14(-7)$ & 7.26 & \\
\hline $0014+098$ & & 13,770 & $(342)$ & $7.96(0.05)$ & $0.59(0.03)$ & 11.34 & -2.24 & 15.87 & 80 & $1.63(-6)$ & 8.46 & 1,2 \\
\hline $0017+061$ & PHL 790 & 28,060 & ( 459) & $7.76(0.06)$ & $0.53(0.03)$ & 9.70 & -0.85 & 14.75 & 102 & $1.48(-7)$ & 7.12 & 2 \\
\hline $0033+016$ & $\mathrm{G} 1-7$ & 10,980 & $(158)$ & $8.83(0.05)$ & $1.12(0.02)$ & 13.28 & -3.23 & 15.61 & 29 & $2.36(-5)$ & 9.32 & \\
\hline $0048+202$ & & 20,160 & ( 312$)$ & $7.99(0.05)$ & $0.62(0.03)$ & 10.70 & -1.59 & 14.85 & 67 & $5.80(-7)$ & 7.93 & \\
\hline $0052+190$ & & 29,420 & $(442)$ & $7.72(0.05)$ & $0.51(0.02)$ & 9.52 & -0.74 & 16.51 & 249 & $1.18(-7)$ & 7.03 & \\
\hline $0059+258$ & & 21,370 & (411) & $8.04(0.06)$ & $0.65(0.03)$ & 10.68 & -1.52 & 15.69 & 100 & $5.52(-7)$ & 7.89 & \\
\hline $0101+048$ & G1-45 & 8530 & ( 123$)$ & $8.27(0.05)$ & $0.77(0.04)$ & 13.17 & -3.27 & 14.03 & 14 & $2.20(-5)$ & 9.18 & \\
\hline $0102+096$ & PHL 972 & 24,770 & $(374)$ & $7.93(0.05)$ & $0.60(0.02)$ & 10.23 & -1.18 & 14.46 & 70 & $2.95(-7)$ & 7.45 & \\
\hline $0107+268$ & GD 12 & 13,880 & $(242)$ & $7.87(0.05)$ & $0.54(0.03)$ & 11.19 & -2.18 & 15.03 & 58 & $1.33(-6)$ & 8.39 & \\
\hline $0125+094$ & PB 6456 & 32,020 & (503) & $7.95(0.06)$ & $0.63(0.03)$ & 9.71 & -0.74 & 16.28 & 205 & $1.44(-7)$ & 6.94 & 2 \\
\hline $0126+101$ & $\mathrm{G} 2-40$ & 8690 & ( 125$)$ & $7.84(0.06)$ & $0.51(0.03)$ & 12.45 & -2.98 & 14.38 & 24 & $8.26(-6)$ & 8.89 & \\
\hline $0127+270$ & GD 14 & 24,870 & ( 388$)$ & $7.83(0.05)$ & $0.55(0.02)$ & 10.08 & -1.12 & 15.96 & 150 & $2.44(-7)$ & 7.36 & \\
\hline $0132+254$ & & 19,960 & ( 351$)$ & $7.45(0.05)$ & $0.41(0.02)$ & 9.84 & -1.25 & 16.12 & 180 & $1.96(-7)$ & 7.80 & \\
\hline $0134+181$ & & 56,380 & ( 1677$)$ & $7.40(0.11)$ & $0.49(0.03)$ & 7.85 & +0.68 & 17.19 & 737 & $1.87(-8)$ & 5.96 & 3 \\
\hline $0136+251$ & & 39,640 & $(711)$ & $8.99(0.07)$ & $1.20(0.03)$ & 11.26 & -1.12 & 16.12 & 93 & $9.24(-7)$ & 7.88 & \\
\hline $0136+152$ & & 7980 & ( 114) & $8.20(0.05)$ & $0.72(0.03)$ & 13.31 & -3.34 & 14.94 & 21 & $2.74(-5)$ & 9.21 & \\
\hline $0143+217$ & G94-9 & 9290 & (133) & $8.49(0.05)$ & $0.92(0.03)$ & 13.22 & -3.27 & 15.05 & 23 & $2.26(-5)$ & 9.28 & \\
\hline $0156+015$ & PB 6544 & 24,120 & $(365)$ & $8.06(0.05)$ & $0.67(0.03)$ & 10.48 & -1.31 & 17.05 & 206 & $4.07(-7)$ & 7.67 & \\
\hline $0205+134$ & & 57,400 & ( 1305$)$ & $7.63(0.08)$ & $0.56(0.03)$ & 8.32 & +0.55 & 14.761 & 194 & $2.92(-8)$ & 6.18 & 4 \\
\hline $0216+144$ & & 26,900 & ( 398$)$ & $7.84(0.05)$ & $0.56(0.02)$ & 9.92 & -0.98 & 14.55 & 84 & $1.95(-7)$ & 7.21 & \\
\hline $0229+271$ & LP $354-382$ & 24,160 & ( 368$)$ & $7.90(0.05)$ & $0.58(0.02)$ & 10.24 & -1.21 & 15.58 & 117 & $3.01(-7)$ & 7.48 & \\
\hline $0237+242$ & & 69,160 & ( 1914) & $7.02(0.09)$ & $0.40(0.02)$ & 6.98 & +1.33 & 16.71 & 881 & $9.32(-9)$ & 7.20 & 2 \\
\hline $0243+155$ & & 16,670 & $(272)$ & $8.02(0.05)$ & $0.63(0.03)$ & 11.08 & -1.94 & 16.51 & 122 & $1.04(-6)$ & 8.25 & \\
\hline $0805+655$ & & 46,650 & (1408) & $7.86(0.12)$ & $0.62(0.05)$ & 8.99 & -0.00 & 16.55 & 324 & $5.95(-8)$ & 6.43 & 1,2 \\
\hline $0808+595$ & & 27,330 & $(436)$ & $7.40(0.05)$ & $0.42(0.02)$ & 9.12 & -0.64 & 16.01 & 238 & $7.66(-8)$ & 7.07 & \\
\hline $0812+478$ & & 60,920 & (1220) & $7.58(0.07)$ & $0.55(0.02)$ & 8.15 & +0.70 & 15.22 & 259 & $2.47(-8)$ & 6.06 & 4,5 \\
\hline $0814+569$ & & 37,650 & $(561)$ & $7.89(0.05)$ & $0.61(0.02)$ & 9.33 & -0.41 & 16.64 & 290 & $8.86(-8)$ & 6.68 & \\
\hline
\end{tabular}


Table 1-Continued

\begin{tabular}{|c|c|c|c|c|c|c|c|c|c|c|c|c|}
\hline $0816+297$ & & 16,660 & $(267)$ & $7.84(0.05)$ & $0.53(0.03)$ & 10.81 & -1.83 & 15.93 & 105 & $7.29(-7)$ & 8.11 & \\
\hline $0817+386$ & & 25,230 & $(384)$ & $7.97(0.05)$ & $0.62(0.03)$ & 10.25 & -1.17 & 15.69 & 122 & $3.01(-7)$ & 7.45 & \\
\hline $0819+364$ & & 18,740 & ( 297) & $8.03(0.05)$ & $0.64(0.03)$ & 10.89 & -1.74 & 16.35 & 123 & $7.70(-7)$ & 8.09 & \\
\hline $0821+633$ & & 16,770 & $(281)$ & $7.82(0.05)$ & $0.52(0.03)$ & 10.78 & -1.81 & 15.85 & 103 & $6.93(-7)$ & 8.09 & \\
\hline $0823+317$ & TON 320 & 68,850 & ( 2074) & $7.68(0.12)$ & $0.61(0.04)$ & 8.07 & +0.85 & 15.74 & 342 & $2.25(-8)$ & 5.95 & 3 \\
\hline $0824+289$ & & 50,700 & ( 1288$)$ & $7.74(0.08)$ & $0.58(0.03)$ & 8.67 & +0.24 & 14.27 & 131 & $4.20(-8)$ & 6.36 & 1,4 \\
\hline $0826+455$ & GD 91 & 10,370 & $(151)$ & $7.86(0.05)$ & $0.52(0.03)$ & 11.82 & -2.68 & 15.09 & 45 & $3.39(-6)$ & 8.71 & \\
\hline $0834+501$ & & 60,350 & (2096) & $7.11(0.13)$ & $0.40(0.03)$ & 7.21 & +1.01 & 15.24 & 403 & $1.10(-8)$ & 7.17 & 3,5 \\
\hline $0841+603$ & & 33,170 & $(509)$ & $7.91(0.06)$ & $0.61(0.03)$ & 9.57 & -0.65 & 16.14 & 205 & $1.21(-7)$ & 6.87 & 2 \\
\hline $0843+517$ & & 23,870 & $(392)$ & $7.90(0.05)$ & $0.58(0.03)$ & 10.26 & -1.23 & 16.15 & 151 & $3.09(-7)$ & 7.50 & \\
\hline $0846+558$ & & 27,380 & $(406)$ & $7.88(0.05)$ & $0.58(0.02)$ & 9.94 & -0.97 & 16.42 & 197 & $2.00(-7)$ & 7.19 & \\
\hline $0846+249$ & TON 353 & 66,110 & ( 2534) & $7.11(0.19)$ & $0.40(0.05)$ & 7.02 & +1.17 & 16.71 & 865 & $9.54(-9)$ & 7.15 & 3 \\
\hline $0852+659$ & & 19,070 & ( 299) & $8.13(0.05)$ & $0.70(0.03)$ & 11.00 & -1.77 & 15.84 & 92 & $8.80(-7)$ & 8.15 & 2 \\
\hline $0854+405$ & GD 98 & 22,250 & $(340)$ & $7.91(0.05)$ & $0.58(0.02)$ & 10.40 & -1.36 & 14.90 & 79 & $3.82(-7)$ & 7.66 & \\
\hline $0858+363$ & GD 99 & 11,830 & $(174)$ & $8.09(0.05)$ & $0.66(0.03)$ & 11.78 & -2.58 & 14.544 & 35 & $3.14(-6)$ & 8.70 & 1,6 \\
\hline $0901+140$ & & 9220 & ( 134) & $8.29(0.05)$ & $0.79(0.04)$ & 12.91 & -3.14 & 16.04 & 42 & $1.49(-5)$ & 9.10 & \\
\hline $0904+512$ & & 32,290 & $(485)$ & $8.25(0.05)$ & $0.80(0.03)$ & 10.17 & -0.92 & 16.40 & 176 & $2.49(-7)$ & 7.21 & 5 \\
\hline $0908+171$ & & 17,340 & $(277)$ & $7.92(0.05)$ & $0.57(0.03)$ & 10.86 & -1.81 & 16.11 & 112 & $7.59(-7)$ & 8.11 & \\
\hline $0913+205$ & & 23,610 & (445) & $8.22(0.06)$ & $0.76(0.04)$ & 10.77 & -1.45 & 16.87 & 165 & $5.98(-7)$ & 7.90 & 1 \\
\hline $0913+442$ & G116-16 & 8680 & ( 125$)$ & $8.20(0.05)$ & $0.72(0.03)$ & 12.99 & -3.19 & 15.35 & 29 & $1.69(-5)$ & 9.11 & \\
\hline $0915+526$ & & 15,560 & $(246)$ & $7.96(0.05)$ & $0.60(0.03)$ & 11.12 & -2.03 & 15.49 & 75 & $1.13(-6)$ & 8.30 & \\
\hline $0915+201$ & LB 3016 & 69,970 & (2120) & $7.33(0.10)$ & $0.51(0.03)$ & 7.48 & +1.15 & 16.69 & 694 & $1.36(-8)$ & 5.54 & \\
\hline $0916+065$ & & 43,960 & $(760)$ & $7.72(0.06)$ & $0.56(0.02)$ & 8.83 & -0.01 & 16. & 271 & $5.02(-8)$ & 6.51 & 2 \\
\hline $0920+217$ & LB 3025 & 18,000 & $(282)$ & $7.83(0.05)$ & $0.53(0.02)$ & 10.67 & -1.69 & 16.55 & 150 & $5.83(-7)$ & 7.99 & \\
\hline $0920+364$ & TON 1054 & 24,060 & $(405)$ & $7.63(0.05)$ & $0.46(0.02)$ & 9.84 & -1.05 & 16. & 171 & $1.84(-7)$ & 7.33 & \\
\hline $0921+355$ & G117-B15A & 11,630 & $(172)$ & $7.98(0.05)$ & $0.59(0.03)$ & 11.66 & -2.55 & 15.53 & 59 & $2.69(-6)$ & 8.65 & 1,6 \\
\hline $0922+162$ & & 23,560 & $(369)$ & $8.35(0.05)$ & $0.85(0.03)$ & 10.99 & -1.54 & 16.19 & 109 & $7.96(-7)$ & 8.01 & 7 \\
\hline $0922+183$ & & 24,730 & $(428)$ & $8.21(0.06)$ & $0.76(0.04)$ & 10.67 & -1.36 & 16.51 & 147 & $5.15(-7)$ & 7.81 & \\
\hline $0928+085$ & & 29,430 & (437) & $8.56(0.05)$ & $0.98(0.03)$ & 10.90 & -1.30 & 16.99 & 165 & $6.36(-7)$ & 7.86 & \\
\hline $0933+026$ & & 22,360 & $(401)$ & $8.04(0.05)$ & $0.65(0.03)$ & 10.59 & -1.43 & 16.04 & 123 & $4.82(-7)$ & 7.80 & 1 \\
\hline
\end{tabular}


Table 1-Continued

\begin{tabular}{|c|c|c|c|c|c|c|c|c|c|c|c|c|}
\hline PG & Name & $T_{\text {eff }}$ & $(\mathrm{K})$ & $\log g$ & $M / M_{\odot}$ & $M_{V}$ & $\log L / L_{\odot}$ & $V$ & $D(\mathrm{pc})$ & $1 / V_{\max }$ & $\log \tau$ & Notes \\
\hline $0933+729$ & & 17,380 & $(267)$ & $8.00(0.05)$ & $0.62(0.03)$ & 10.97 & -1.85 & 15.71 & 88 & $8.85(-7)$ & 8.17 & \\
\hline $0934+338$ & TON 1080 & 24,380 & $(376)$ & $7.24(0.05)$ & $0.38(0.01)$ & 9.05 & -0.72 & 16.35 & 288 & $7.27(-8)$ & 7.34 & \\
\hline $0937+506$ & & 35,830 & (528) & $7.88(0.05)$ & $0.60(0.02)$ & 9.38 & -0.49 & 16.00 & 211 & $9.49(-8)$ & 6.75 & \\
\hline $0938+286$ & TON 20 & 14,490 & ( 243) & $7.82(0.05)$ & $0.52(0.02)$ & 11.04 & -2.07 & 15.53 & 79 & $1.06(-6)$ & 8.30 & \\
\hline $0938+299$ & TON 443 & 19,750 & ( 311$)$ & $8.14(0.05)$ & $0.71(0.03)$ & 10.97 & -1.72 & 16.08 & 105 & $8.31(-7)$ & 8.12 & \\
\hline $0938+550$ & & 18,530 & ( 303$)$ & $8.10(0.05)$ & $0.68(0.03)$ & 11.02 & -1.80 & 14.82 & 57 & $9.09(-7)$ & 8.17 & \\
\hline $0939+262$ & TON 21 & 67,930 & $(1366)$ & $7.82(0.07)$ & $0.65(0.03)$ & 8.48 & +0.71 & 14.65 & 171 & $3.40(-8)$ & 5.98 & \\
\hline $0941+433$ & US 906 & 25,680 & ( 403) & $7.92(0.05)$ & $0.60(0.03)$ & 10.14 & -1.11 & 16.37 & 175 & $2.62(-7)$ & 7.36 & \\
\hline $0943+441$ & G116-52 & 12,820 & ( 248) & $7.55(0.05)$ & $0.41(0.02)$ & 10.84 & -2.11 & 13.29 & 30 & $8.72(-7)$ & 8.53 & \\
\hline $0945+246$ & LB 11146 & 14,500 & ( 1021) & $8.50(0.11)$ & $0.93(0.07)$ & 12.08 & -2.49 & 14.37 & 28 & $4.34(-6)$ & 8.78 & 7,8 \\
\hline $0947+326$ & TON 458 & 22,060 & $(341)$ & $8.31(0.05)$ & $0.82(0.03)$ & 11.04 & -1.63 & 15.43 & 75 & $8.72(-7)$ & 8.07 & \\
\hline $0948+534$ & & 130,370 & ( 6603$)$ & $7.26(0.12)$ & $0.67(0.03)$ & 6.42 & +2.42 & 15.08 & 540 & $6.44(-9)$ & 4.37 & 4,9 \\
\hline $0949+256$ & TON 462 & 22,600 & $(356)$ & $7.98(0.05)$ & $0.62(0.03)$ & 10.48 & -1.38 & 16.31 & 146 & $4.21(-7)$ & 7.71 & 7 \\
\hline $0950+186$ & & 31,790 & $(575)$ & $7.68(0.09)$ & $0.50(0.03)$ & 9.29 & -0.57 & 15.35 & 162 & $8.85(-8)$ & 6.92 & 1 \\
\hline $0950+024$ & & 40,330 & (689) & $7.80(0.06)$ & $0.58(0.03)$ & 9.07 & -0.22 & 16.82 & 355 & $6.58(-8)$ & 6.60 & \\
\hline $0950+139$ & & 108,390 & (16786) & $7.39(0.38)$ & $0.64(0.31)$ & 6.97 & +1.95 & 16.025 & 645 & $9.11(-9)$ & 5.13 & 9 \\
\hline $0950+078$ & & 14,770 & $(254)$ & $7.95(0.05)$ & $0.59(0.03)$ & 11.19 & -2.12 & 16.09 & 95 & $1.29(-6)$ & 8.36 & 2 \\
\hline $0954+697$ & & 21,420 & ( 355$)$ & $7.91(0.05)$ & $0.58(0.03)$ & 10.47 & -1.43 & 15.95 & 124 & $4.20(-7)$ & 7.73 & \\
\hline $0954+248$ & $\mathrm{G} 49-33$ & 8620 & $(126)$ & $8.30(0.06)$ & $0.79(0.04)$ & 13.18 & -3.27 & 15.3 & 26 & $2.20(-5)$ & 9.18 & 2 \\
\hline $0955-008$ & LB 3090 & 23,120 & (418) & $7.99(0.06)$ & $0.63(0.03)$ & 10.45 & -1.34 & 16.77 & 183 & $4.00(-7)$ & 7.67 & \\
\hline $0956+046$ & & 18,150 & ( 304$)$ & $7.81(0.05)$ & $0.52(0.03)$ & 10.62 & -1.66 & 15.85 & 111 & $5.47(-7)$ & 7.96 & \\
\hline $0956+021$ & & 15,670 & ( 248) & $7.80(0.05)$ & $0.51(0.02)$ & 10.87 & -1.92 & 15.54 & 85 & $8.14(-7)$ & 8.18 & \\
\hline $1000-002$ & LB 564 & 19,470 & $(312)$ & $7.99(0.05)$ & $0.62(0.03)$ & 10.76 & -1.64 & 16.13 & 118 & $6.32(-7)$ & 7.99 & \\
\hline $1001+204$ & TON 1150 & 21,490 & ( 558$)$ & $7.97(0.08)$ & $0.61(0.04)$ & 10.56 & -1.46 & 15.35 & 90 & $4.72(-7)$ & 7.81 & 1 \\
\hline $1003-023$ & & 20,340 & $(324)$ & $7.95(0.05)$ & $0.60(0.03)$ & 10.62 & -1.54 & 15.43 & 91 & $5.23(-7)$ & 7.88 & \\
\hline $1005+642$ & GD 462 & 19,660 & $(302)$ & $7.93(0.05)$ & $0.58(0.03)$ & 10.65 & -1.59 & 13.74 & 41 & $5.48(-7)$ & 7.91 & 5 \\
\hline $1010+065$ & & 44,450 & ( 866$)$ & $7.89(0.07)$ & $0.63(0.03)$ & 9.10 & -0.11 & 16.57 & 312 & $6.72(-8)$ & 6.48 & \\
\hline $1013+257$ & TON 494 & 21,990 & (339) & $8.00(0.05)$ & $0.63(0.03)$ & 10.57 & -1.44 & 15.97 & 120 & $4.72(-7)$ & 7.79 & 2 \\
\hline $1015+161$ & & 19,540 & $(305)$ & $8.04(0.05)$ & $0.65(0.03)$ & 10.83 & -1.67 & 15.62 & 90 & $6.94(-7)$ & 8.04 & \\
\hline $1017+125$ & & 21,670 & $(340)$ & $7.94(0.05)$ & $0.60(0.03)$ & 10.50 & -1.43 & 15.8 & 114 & $4.37(-7)$ & 7.75 & \\
\hline
\end{tabular}


Table 1-Continued

\begin{tabular}{|c|c|c|c|c|c|c|c|c|c|c|c|c|}
\hline PG & Name & $T_{\text {eff }}$ & $(\mathrm{K})$ & $\log g$ & $M / M_{\odot}$ & $M_{V}$ & $\log L / L_{\odot}$ & $V$ & $D(\mathrm{pc})$ & $1 / V_{\max }$ & $\log \tau$ & Notes \\
\hline $1018+411$ & & 23,680 & ( 397$)$ & $8.04(0.05)$ & $0.66(0.03)$ & 10.49 & -1.33 & 15.57 & 103 & $4.17(-7)$ & 7.69 & \\
\hline $1019+129$ & & 18,020 & ( 299) & $8.00(0.05)$ & $0.62(0.03)$ & 10.91 & -1.79 & 15.6 & 86 & $8.05(-7)$ & 8.12 & \\
\hline $1022+050$ & LP $550-52$ & 11,680 & ( 179$)$ & $7.64(0.06)$ & $0.44(0.02)$ & 11.17 & -2.34 & 14.20 & 40 & $1.40(-6)$ & 8.69 & \\
\hline $1023+009$ & & 35,950 & $(565)$ & $7.83(0.06)$ & $0.58(0.03)$ & 9.30 & -0.45 & 16.45 & 269 & $8.66(-8)$ & 6.74 & \\
\hline $1026+002$ & & 17,180 & $(256)$ & $7.97(0.04)$ & $0.60(0.03)$ & 10.95 & -1.86 & 13.88 & 38 & $8.60(-7)$ & 8.16 & 1 \\
\hline $1026+024$ & LP 550-292 & 12,570 & ( 187$)$ & $7.98(0.05)$ & $0.60(0.03)$ & 11.52 & -2.41 & 14.25 & 35 & $2.17(-6)$ & 8.56 & \\
\hline $1026+454$ & & 35,720 & $(531)$ & $7.90(0.05)$ & $0.61(0.02)$ & 9.42 & -0.51 & 16.12 & 218 & $1.00(-7)$ & 6.76 & \\
\hline $1031+234$ & TON 527 & 15,000 & $(1022)$ & $8.50(0.30)$ & $0.93(0.18)$ & 12.02 & -2.43 & 15.84 & 58 & $3.94(-6)$ & 8.74 & 10 \\
\hline $1031+063$ & & 20,750 & $(354)$ & $7.87(0.05)$ & $0.56(0.03)$ & 10.47 & -1.46 & 16.31 & 147 & $4.25(-7)$ & 7.76 & \\
\hline $1033+464$ & GD 123 & 29,430 & $(434)$ & $7.88(0.05)$ & $0.58(0.02)$ & 9.77 & -0.84 & 14.32 & 81 & $1.59(-7)$ & 7.06 & 1 \\
\hline $1034+492$ & GD 304 & 20,650 & ( 340$)$ & $8.17(0.05)$ & $0.73(0.03)$ & 10.94 & -1.66 & 15.46 & 80 & $7.84(-7)$ & 8.07 & \\
\hline $1036+086$ & & 22,230 & $(342)$ & $7.49(0.05)$ & $0.42(0.02)$ & 9.72 & -1.08 & 16.26 & 203 & $1.63(-7)$ & 7.66 & 2 \\
\hline $1037+512$ & & 20,100 & ( 336$)$ & $8.03(0.05)$ & $0.64(0.03)$ & 10.76 & -1.62 & 16.25 & 125 & $6.31(-7)$ & 7.98 & 1,5 \\
\hline $1038+634$ & & 24,450 & $(365)$ & $8.38(0.05)$ & $0.87(0.03)$ & 10.97 & -1.50 & 15.15 & 68 & $7.60(-7)$ & 7.98 & 5 \\
\hline $1039+748$ & & 29,100 & (463) & $7.74(0.06)$ & $0.52(0.03)$ & 9.59 & -0.78 & 15.83 & 176 & $1.29(-7)$ & 7.05 & 2 \\
\hline $1040+452$ & & 45,230 & ( 1023) & $7.96(0.09)$ & $0.67(0.04)$ & 9.20 & -0.13 & 16.955 & 354 & $7.52(-8)$ & 6.47 & 4 \\
\hline $1041+580$ & & 30,440 & $(445)$ & $7.75(0.05)$ & $0.53(0.02)$ & 9.50 & -0.70 & 14.59 & 104 & $1.14(-7)$ & 6.99 & \\
\hline $1046+282$ & TON 547 & 12,610 & ( 202) & $7.97(0.05)$ & $0.59(0.03)$ & 11.51 & -2.40 & 15.40 & 60 & $2.15(-6)$ & 8.56 & \\
\hline $1047+694$ & & 33,160 & $(504)$ & $8.02(0.06)$ & $0.67(0.03)$ & 9.75 & -0.72 & 16.05 & 181 & $1.49(-7)$ & 6.92 & 2 \\
\hline $1049+103$ & & 20,550 & (337) & $7.91(0.05)$ & $0.58(0.03)$ & 10.55 & -1.50 & 15.7 & 107 & $4.71(-7)$ & 7.82 & 1 \\
\hline $1051+274$ & GD 125 & 23,100 & $(352)$ & $8.37(0.05)$ & $0.86(0.03)$ & 11.06 & -1.59 & 14.15 & 41 & $8.71(-7)$ & 8.05 & \\
\hline $1057+719$ & & 41,180 & ( 636$)$ & $7.75(0.05)$ & $0.56(0.02)$ & 8.97 & -0.15 & 14.8 & 146 & $5.86(-8)$ & 6.58 & \\
\hline $1058-129$ & & 24,310 & $(354)$ & $8.71(0.04)$ & $1.06(0.02)$ & 11.57 & -1.75 & 15.75 & 68 & $1.66(-6)$ & 8.26 & \\
\hline $1100+605$ & G197-4 & 17,920 & ( 271) & $8.02(0.05)$ & $0.63(0.03)$ & 10.94 & -1.81 & 13.8 & 37 & $8.39(-7)$ & 8.14 & \\
\hline $1101+364$ & & 13,040 & $(230)$ & $7.24(0.05)$ & $0.32(0.01)$ & 10.30 & -1.88 & 14.49 & 68 & $4.22(-7)$ & 8.35 & 7 \\
\hline $1101+242$ & TON 53 & 30,700 & ( 481) & $7.83(0.06)$ & $0.56(0.03)$ & 9.60 & -0.73 & 16.52 & 241 & $1.28(-7)$ & 6.98 & \\
\hline $1102+749$ & GD 466 & 19,710 & ( 315$)$ & $8.36(0.05)$ & $0.85(0.03)$ & 11.31 & -1.86 & 14.97 & 53 & $1.31(-6)$ & 8.26 & \\
\hline $1105-049$ & G163-50 & 15,140 & ( 231) & $7.85(0.05)$ & $0.53(0.02)$ & 11.00 & -2.01 & 13.06 & 25 & $9.80(-7)$ & 8.26 & 1 \\
\hline $1108+325$ & TON 60 & 62,950 & ( 2098) & $7.59(0.12)$ & $0.56(0.04)$ & 8.13 & +0.75 & 16.85 & 554 & $2.42(-8)$ & 6.04 & \\
\hline $1108+476$ & GD 129 & 12,400 & $(214)$ & $8.31(0.05)$ & $0.80(0.04)$ & 12.02 & -2.64 & 15.38 & 46 & $4.27(-6)$ & 8.78 & \\
\hline
\end{tabular}


Table 1-Continued

\begin{tabular}{|c|c|c|c|c|c|c|c|c|c|c|c|c|}
\hline$P G$ & Name & $T_{\text {eff }}$ & $(\mathrm{K})$ & $\log g$ & $M / M_{\odot}$ & $M_{V}$ & $\log L / L_{\odot}$ & $V$ & $D(\mathrm{pc})$ & $1 / V_{\max }$ & $\log \tau$ & Notes \\
\hline $1109+244$ & TON 61 & 37,840 & ( 601$)$ & $8.13(0.05)$ & $0.74(0.03)$ & 9.72 & -0.56 & 15.773 & 162 & $1.39(-7)$ & 6.75 & \\
\hline $1113+413$ & & 25,440 & ( 375$)$ & $7.83(0.05)$ & $0.55(0.02)$ & 10.02 & -1.07 & 15.43 & 120 & $2.26(-7)$ & 7.31 & \\
\hline $1115+166$ & & 22,090 & ( 311$)$ & $8.12(0.04)$ & $0.70(0.03)$ & 10.74 & -1.50 & 15.15 & 76 & $5.88(-7)$ & 7.91 & 11 \\
\hline $1116+026$ & GD 133 & 12,290 & ( 203) & $8.05(0.05)$ & $0.63(0.03)$ & 11.65 & -2.49 & 14.57 & 38 & $2.61(-6)$ & 8.63 & \\
\hline $1122+546$ & GD 307 & 14,380 & ( 231) & $7.83(0.05)$ & $0.52(0.02)$ & 11.07 & -2.09 & 15.43 & 74 & $1.10(-6)$ & 8.32 & \\
\hline $1124-019$ & & 24,280 & $(387)$ & $7.72(0.05)$ & $0.50(0.02)$ & 9.95 & -1.09 & 16.52 & 205 & $2.12(-7)$ & 7.35 & \\
\hline $1125+175$ & & 55,030 & ( 2027) & $7.72(0.14)$ & $0.58(0.05)$ & 8.54 & +0.40 & 16.81 & 450 & $3.65(-8)$ & 6.27 & 4 \\
\hline $1125-026$ & & 31,090 & (459) & $8.19(0.05)$ & $0.76(0.03)$ & 10.15 & -0.94 & 15.42 & 113 & $2.44(-7)$ & 7.23 & \\
\hline $1126+384$ & GD 310 & 25,150 & ( 383$)$ & $7.90(0.05)$ & $0.59(0.02)$ & 10.16 & -1.14 & 14.914 & 89 & $2.69(-7)$ & 7.39 & \\
\hline $1133+293$ & Feige 45 & 23,030 & $(366)$ & $7.84(0.05)$ & $0.55(0.02)$ & 10.23 & -1.25 & 15. & 90 & $3.03(-7)$ & 7.52 & \\
\hline $1134+301$ & GD 140 & 21,280 & ( 325$)$ & $8.55(0.05)$ & $0.96(0.03)$ & 11.50 & -1.86 & 12.47 & 15 & $1.62(-6)$ & 8.30 & \\
\hline $1141+505$ & LB 2094 & 20,760 & ( 357$)$ & $7.91(0.05)$ & $0.58(0.03)$ & 10.52 & -1.48 & 16.62 & 165 & $4.57(-7)$ & 7.80 & \\
\hline $1141+078$ & & 62,810 & ( 1303$)$ & $7.52(0.07)$ & $0.54(0.02)$ & 8.00 & +0.80 & 14.57 & 206 & $2.14(-8)$ & 5.97 & 2 \\
\hline $1143+321$ & G148-7 & 14,940 & $(231)$ & $7.93(0.05)$ & $0.57(0.03)$ & 11.14 & -2.08 & 13.65 & 31 & $1.19(-6)$ & 8.32 & 1 \\
\hline $1145+188$ & & 26,570 & ( 392$)$ & $7.82(0.05)$ & $0.55(0.02)$ & 9.91 & -0.99 & 14.22 & 72 & $1.95(-7)$ & 7.22 & \\
\hline $1147+256$ & G121-22 & 10,200 & ( 147$)$ & $8.14(0.05)$ & $0.69(0.03)$ & 12.29 & -2.88 & 15.67 & 47 & $6.40(-6)$ & 8.90 & \\
\hline $1149+411$ & GD 312 & 14,070 & $(271)$ & $7.84(0.05)$ & $0.53(0.03)$ & 11.12 & -2.13 & 16.08 & 98 & $1.21(-6)$ & 8.35 & \\
\hline $1149+058$ & & 11,070 & ( 161$)$ & $8.15(0.05)$ & $0.69(0.03)$ & 12.04 & -2.73 & 14.96 & 38 & $4.52(-6)$ & 8.81 & \\
\hline $1158+433$ & & 14,050 & ( 229) & $7.85(0.05)$ & $0.53(0.02)$ & 11.13 & -2.14 & 16.17 & 101 & $1.22(-6)$ & 8.35 & 2 \\
\hline
\end{tabular}


Table 1-Continued

\begin{tabular}{|c|c|c|c|c|c|c|c|c|c|c|c|c|}
\hline PG & Name & $T_{\text {eff }}$ & $(\mathrm{K})$ & $\log g$ & $M / M_{\odot}$ & $M_{V}$ & $\log L / L_{\odot}$ & $V$ & $D(\mathrm{pc})$ & $1 / V_{\max }$ & $\log \tau$ & Notes \\
\hline $1202+309$ & TON 75 & 28,880 & ( 457) & $7.80(0.06)$ & $0.55(0.03)$ & 9.70 & -0.83 & 15. & 114 & $1.46(-7)$ & 7.07 & \\
\hline $1204+451$ & & 22,560 & ( 369$)$ & $7.79(0.05)$ & $0.52(0.02)$ & 10.20 & -1.26 & 14.97 & 90 & $2.92(-7)$ & 7.53 & 1,2 \\
\hline $1210+141$ & & 31,930 & $(475)$ & $7.03(0.05)$ & $0.34(0.01)$ & 8.10 & -0.09 & 14.712 & 209 & $2.52(-8)$ & 7.60 & 4 \\
\hline $1210+464$ & & 27,670 & ( 665$)$ & $7.85(0.10)$ & $0.57(0.05)$ & 9.87 & -0.93 & 15.79 & 153 & $1.81(-7)$ & 7.17 & 1,2 \\
\hline $1210+533$ & & 44,820 & ( 738) & $7.89(0.05)$ & $0.63(0.03)$ & 9.03 & -0.10 & 14.12 & 104 & $6.16(-8)$ & 6.47 & 3 \\
\hline $1214+268$ & LB 2 & 65,700 & ( 1695$)$ & $7.67(0.12)$ & $0.60(0.04)$ & 8.13 & +0.76 & 15.63 & 315 & $2.40(-8)$ & 6.01 & 3 \\
\hline $1216+036$ & & 13,800 & $(322)$ & $7.85(0.06)$ & $0.53(0.03)$ & 11.17 & -2.18 & 15.99 & 91 & $1.31(-6)$ & 8.39 & \\
\hline $1218+497$ & LB 2318 & 35,660 & ( 567$)$ & $7.87(0.06)$ & $0.60(0.03)$ & 9.37 & -0.49 & 16.24 & 236 & $9.44(-8)$ & 6.76 & \\
\hline $1220+234$ & TON 610 & 26,540 & ( 429) & $8.28(0.06)$ & $0.81(0.04)$ & 10.63 & -1.28 & 15.64 & 100 & $4.77(-7)$ & 7.74 & 12 \\
\hline $1223+478$ & & 30,980 & (463) & $7.84(0.05)$ & $0.57(0.03)$ & 9.60 & -0.72 & 16.69 & 262 & $1.27(-7)$ & 6.96 & \\
\hline $1224+309$ & & 28,820 & $(480)$ & $7.38(0.06)$ & $0.42(0.02)$ & 8.97 & -0.53 & 16.15 & 272 & $6.34(-8)$ & 7.08 & \\
\hline $1229-013$ & & 19,430 & ( 298) & $7.47(0.05)$ & $0.41(0.01)$ & 9.92 & -1.31 & 13.79 & 59 & $2.20(-7)$ & 7.85 & \\
\hline $1230+418$ & HZ 28 & 19,520 & (298) & $7.98(0.05)$ & $0.61(0.03)$ & 10.74 & -1.64 & 15.73 & 99 & $6.19(-7)$ & 7.98 & \\
\hline $1232+479$ & GD 148 & 14,370 & ( 235$)$ & $7.82(0.05)$ & $0.52(0.02)$ & 11.06 & -2.09 & 14.50 & 48 & $1.09(-6)$ & 8.31 & \\
\hline $1233+338$ & & 19,020 & (304) & $7.99(0.05)$ & $0.62(0.03)$ & 10.80 & -1.69 & 15.79 & 99 & $6.79(-7)$ & 8.03 & 2 \\
\hline $1234+482$ & & 55,040 & $(975)$ & $7.78(0.06)$ & $0.61(0.02)$ & 8.66 & +0.35 & 14.45 & 144 & $4.12(-8)$ & 6.24 & 4 \\
\hline $1237-029$ & LP $615-183$ & 10,240 & ( 147$)$ & $8.58(0.05)$ & $0.98(0.03)$ & 13.02 & -3.16 & 15.97 & 38 & $1.69(-5)$ & 9.22 & \\
\hline $1240+754$ & LB 261 & 18,750 & $(322)$ & $7.83(0.05)$ & $0.53(0.03)$ & 10.59 & -1.62 & 16.2 & 132 & $5.17(-7)$ & 7.92 & 1 \\
\hline $1241+235$ & LB 16 & 26,730 & (408) & $7.93(0.05)$ & $0.60(0.03)$ & 10.06 & -1.04 & 15.23 & 108 & $2.33(-7)$ & 7.27 & \\
\hline $1241-010$ & & 23,800 & $(362)$ & $7.33(0.05)$ & $0.40(0.01)$ & 9.26 & -0.83 & 14.00 & 88 & $9.38(-8)$ & 7.36 & 7 \\
\hline $1244+149$ & G61-17 & 10,680 & $(156)$ & $8.06(0.05)$ & $0.64(0.03)$ & 12.02 & -2.75 & 15.9 & 59 & $4.43(-6)$ & 8.80 & \\
\hline $1249+160$ & GD 150 & 25,590 & $(400)$ & $7.29(0.05)$ & $0.39(0.01)$ & 9.03 & -0.67 & 14.615 & 130 & $7.06(-8)$ & 7.24 & 4 \\
\hline $1252+378$ & HZ 34 & 79,900 & $(3765)$ & $6.61(0.21)$ & $0.36(0.04)$ & 5.71 & +1.95 & 15.77 & 1026 & $4.70(-9)$ & 7.44 & 3 \\
\hline $1254+223$ & GD 153 & 39,450 & $(606)$ & $7.85(0.05)$ & $0.60(0.02)$ & 9.19 & -0.30 & 13.368 & 68 & $7.55(-8)$ & 6.62 & \\
\hline $1255+426$ & & 38,310 & ( 1135$)$ & $8.09(0.13)$ & $0.71(0.07)$ & 9.62 & -0.51 & 17.25 & 335 & $1.24(-7)$ & 6.74 & \\
\hline $1257+048$ & GD 267 & 22,320 & ( 348$)$ & $8.01(0.05)$ & $0.63(0.03)$ & 10.54 & -1.41 & 15.02 & 78 & $4.55(-7)$ & 7.76 & 1 \\
\hline $1257+278$ & G149-28 & 8730 & ( 127$)$ & $8.33(0.06)$ & $0.81(0.04)$ & 13.17 & -3.26 & 15.41 & 28 & $2.17(-5)$ & 9.19 & \\
\hline $1257+032$ & PB 4421 & 17,290 & (269) & $7.79(0.05)$ & $0.51(0.02)$ & 10.68 & -1.74 & 15.67 & 99 & $6.00(-7)$ & 8.02 & 5 \\
\hline $1258+593$ & GD 322 & 14,480 & ( 229) & $7.87(0.05)$ & $0.54(0.02)$ & 11.12 & -2.10 & 15.31 & 69 & $1.18(-6)$ & 8.33 & \\
\hline $1301+545$ & LB 248 & 33,560 & $(513)$ & $8.05(0.06)$ & $0.69(0.03)$ & 9.78 & -0.72 & 15.70 & 152 & $1.54(-7)$ & 6.92 & \\
\hline
\end{tabular}


Table 1-Continued

\begin{tabular}{|c|c|c|c|c|c|c|c|c|c|c|c|c|}
\hline PG & Name & $T_{\text {eff }}$ & $(\mathrm{K})$ & $\log g$ & $M / M_{\odot}$ & $M_{V}$ & $\log L / L_{\odot}$ & V & $D(\mathrm{pc})$ & $1 / V_{\max }$ & $\log \tau$ & Notes \\
\hline $1305+018$ & & 28,980 & $(445)$ & $7.87(0.05)$ & $0.58(0.03)$ & 9.79 & -0.86 & 15.08 & 114 & $1.64(-7)$ & 7.08 & \\
\hline $1305-017$ & & 44,410 & $(1526)$ & $7.76(0.11)$ & $0.57(0.05)$ & 8.75 & -0.02 & 17.05 & 457 & $4.60(-8)$ & 6.51 & 3,5 \\
\hline $1307+354$ & GD 154 & 11,180 & $(164)$ & $8.15(0.05)$ & $0.70(0.03)$ & 12.03 & -2.72 & 15.33 & 45 & $4.41(-6)$ & 8.80 & 6 \\
\hline $1310+583$ & & 10,560 & ( 153$)$ & $8.32(0.05)$ & $0.80(0.03)$ & 12.45 & -2.92 & 14.09 & 21 & $7.90(-6)$ & 8.96 & 5 \\
\hline $1314+294$ & $\mathrm{HZ} 43$ & 52,520 & $(1152)$ & $8.03(0.08)$ & $0.71(0.04)$ & 9.14 & +0.10 & 12.99 & 58 & $6.93(-8)$ & 6.24 & 1 \\
\hline $1314-067$ & & 16,270 & ( 269) & $7.88(0.05)$ & $0.55(0.03)$ & 10.92 & -1.90 & 15.89 & 98 & $8.46(-7)$ & 8.18 & 2 \\
\hline $1317+453$ & G177-31 & 13,320 & ( 231) & $7.38(0.05)$ & $0.36(0.02)$ & 10.48 & -1.93 & 14.14 & 54 & $5.27(-7)$ & 8.41 & 7 \\
\hline $1319+466$ & G177-34 & 13,880 & ( 321$)$ & $8.19(0.05)$ & $0.73(0.03)$ & 11.66 & -2.37 & 14.55 & 37 & $2.53(-6)$ & 8.60 & \\
\hline $1320+645$ & & 27,130 & ( 459) & $7.51(0.06)$ & $0.44(0.02)$ & 9.34 & -0.74 & 16.38 & 256 & $9.79(-8)$ & 6.92 & \\
\hline $1322+077$ & & 26,680 & ( 473$)$ & $7.84(0.06)$ & $0.56(0.03)$ & 9.93 & -0.99 & 16.26 & 184 & $1.99(-7)$ & 7.23 & 2 \\
\hline $1325+279$ & & 21,270 & ( 435) & $8.04(0.06)$ & $0.65(0.04)$ & 10.68 & -1.52 & 15.88 & 109 & $5.51(-7)$ & 7.90 & \\
\hline $1325+168$ & & 17,970 & ( 305$)$ & $8.18(0.05)$ & $0.73(0.03)$ & 11.18 & -1.91 & 16.07 & 95 & $1.15(-6)$ & 8.27 & \\
\hline $1327-083$ & Wolf $485 \mathrm{~A}$ & 13,490 & $(247)$ & $7.89(0.05)$ & $0.55(0.03)$ & 11.27 & -2.24 & 12.32 & 16 & $1.51(-6)$ & 8.43 & 1 \\
\hline $1328+344$ & & 16,300 & $(260)$ & $7.90(0.05)$ & $0.56(0.03)$ & 10.94 & -1.91 & 15.45 & 80 & $8.67(-7)$ & 8.19 & 2 \\
\hline $1330+473$ & & 22,460 & ( 344$)$ & $7.95(0.05)$ & $0.60(0.03)$ & 10.44 & -1.37 & 15.20 & 89 & $4.00(-7)$ & 7.68 & \\
\hline $1333+498$ & & 29,260 & $(442)$ & $7.93(0.05)$ & $0.61(0.03)$ & 9.87 & -0.89 & 15.76 & 150 & $1.80(-7)$ & 7.10 & \\
\hline $1333+525$ & LB 2694 & 16,570 & ( 288) & $7.86(0.05)$ & $0.54(0.03)$ & 10.86 & -1.86 & 16.35 & 125 & $7.78(-7)$ & 8.14 & \\
\hline $1335+701$ & & 30,140 & ( 438) & $8.25(0.05)$ & $0.79(0.03)$ & 10.32 & -1.04 & 15.5 & 108 & $3.04(-7)$ & 7.40 & \\
\hline $1335+369$ & & 20,510 & ( 328$)$ & $7.78(0.05)$ & $0.51(0.02)$ & 10.35 & -1.42 & 14.58 & 70 & $3.68(-7)$ & 7.71 & \\
\hline $1337+705$ & G238-44 & 20,460 & ( 306$)$ & $7.90(0.05)$ & $0.57(0.02)$ & 10.54 & -1.50 & 12.79 & 28 & $4.68(-7)$ & 7.82 & \\
\hline $1339+346$ & & 15,960 & $(250)$ & $7.82(0.05)$ & $0.52(0.02)$ & 10.87 & -1.90 & 15.87 & 100 & $8.02(-7)$ & 8.17 & 2 \\
\hline $1342+444$ & & 72,350 & $(2342)$ & $7.84(0.11)$ & $0.67(0.04)$ & 8.45 & +0.82 & 16.65 & 437 & $3.27(-8)$ & 5.92 & 13 \\
\hline $1344+573$ & G223-24 & 13,390 & $(281)$ & $7.94(0.05)$ & $0.58(0.03)$ & 11.36 & -2.28 & 13.35 & 25 & $1.70(-6)$ & 8.47 & \\
\hline $1344+509$ & & 20,330 & ( 325$)$ & $8.11(0.05)$ & $0.69(0.03)$ & 10.86 & -1.64 & 15.95 & 104 & $7.13(-7)$ & 8.04 & 2 \\
\hline $1344+106$ & LHS 2800 & 7140 & ( 108$)$ & $8.12(0.08)$ & $0.67(0.05)$ & 13.63 & -3.49 & 15.08 & 19 & $4.69(-5)$ & 9.28 & \\
\hline $1348+442$ & PB 772 & 19,760 & ( 333$)$ & $8.00(0.05)$ & $0.63(0.03)$ & 10.75 & -1.63 & 16.31 & 129 & $6.27(-7)$ & 7.98 & 2 \\
\hline $1349+552$ & LP $133-144$ & 11,800 & (190) & $7.87(0.06)$ & $0.54(0.03)$ & 11.49 & -2.46 & 16. & 79 & $2.13(-6)$ & 8.58 & 6 \\
\hline $1349+144$ & PB 4117 & 16,620 & $(279)$ & $7.68(0.05)$ & $0.46(0.02)$ & 10.59 & -1.75 & 15.34 & 89 & $5.46(-7)$ & 8.16 & \\
\hline $1350-090$ & LP $907-37$ & 9520 & ( 136$)$ & $8.36(0.05)$ & $0.83(0.03)$ & 12.91 & -3.14 & 14.57 & 21 & $1.47(-5)$ & 9.12 & 14 \\
\hline $1350+657$ & G238-53 & 11,880 & ( 181$)$ & $7.91(0.05)$ & $0.55(0.03)$ & 11.52 & -2.47 & 15.56 & 64 & $2.21(-6)$ & 8.59 & 6 \\
\hline
\end{tabular}


Table 1-Continued

\begin{tabular}{|c|c|c|c|c|c|c|c|c|c|c|c|c|}
\hline$P G$ & Name & $T_{\text {eff }}$ & $(\mathrm{K})$ & $\log g$ & $M / M_{\odot}$ & $M_{V}$ & $\log L / L_{\odot}$ & $V$ & $D(\mathrm{pc})$ & $1 / V_{\max }$ & $\log \tau$ & Notes \\
\hline $1353+409$ & PB 999 & 23,530 & $(364)$ & $7.59(0.05)$ & $0.46(0.02)$ & 9.79 & -1.05 & 15.55 & 142 & $1.74(-7)$ & 7.34 & \\
\hline $1403-077$ & & 50,190 & $(1035)$ & $7.59(0.07)$ & $0.52(0.02)$ & 8.42 & +0.33 & 15.82 & 302 & $3.24(-8)$ & 6.33 & \\
\hline $1407+425$ & & 10,010 & $(143)$ & $8.21(0.05)$ & $0.73(0.03)$ & 12.47 & -2.95 & 15.03 & 32 & $8.15(-6)$ & 8.96 & \\
\hline $1408+324$ & GD 163 & 18,150 & ( 278) & $7.95(0.05)$ & $0.59(0.03)$ & 10.82 & -1.75 & 14.06 & 44 & $7.12(-7)$ & 8.07 & \\
\hline $1410+425$ & PB 1665 & 15,350 & $(264)$ & $7.87(0.05)$ & $0.54(0.03)$ & 11.00 & -2.00 & 16.1 & 104 & $9.76(-7)$ & 8.25 & 2 \\
\hline $1410+318$ & PB 3534 & 19,600 & $(300)$ & $8.01(0.05)$ & $0.63(0.03)$ & 10.78 & -1.65 & 16.64 & 148 & $6.51(-7)$ & 8.00 & \\
\hline $1412-109$ & & 25,700 & $(381)$ & $7.94(0.05)$ & $0.61(0.02)$ & 10.17 & -1.13 & 15.91 & 140 & $2.72(-7)$ & 7.38 & \\
\hline $1413+232$ & G166-14 & 23,370 & ( 414) & $7.72(0.05)$ & $0.50(0.02)$ & 10.03 & -1.16 & 16.41 & 188 & $2.37(-7)$ & 7.43 & \\
\hline $1415+133$ & Feige 93 & 34,270 & ( 499) & $7.38(0.05)$ & $0.42(0.01)$ & 8.61 & -0.22 & 15.37 & 225 & $4.12(-8)$ & 7.04 & \\
\hline $1418-005$ & & 14,290 & (289) & $7.82(0.06)$ & $0.51(0.03)$ & 11.06 & -2.09 & 16.52 & 123 & $1.10(-6)$ & 8.32 & 13 \\
\hline $1421+318$ & TON 197 & 27,190 & $(406)$ & $7.94(0.05)$ & $0.61(0.03)$ & 10.04 & -1.02 & 15.35 & 115 & $2.27(-7)$ & 7.25 & \\
\hline $1422+095$ & GD 165 & 11,980 & ( 178$)$ & $8.06(0.05)$ & $0.64(0.03)$ & 11.72 & -2.55 & 14.32 & 33 & $2.90(-6)$ & 8.67 & 1,6 \\
\hline $1429+373$ & GD 336 & 34,240 & (507) & $8.15(0.05)$ & $0.74(0.03)$ & 9.90 & -0.75 & 15.272 & 118 & $1.77(-7)$ & 6.95 & \\
\hline $1431+154$ & & 13,550 & (436) & $7.95(0.06)$ & $0.58(0.03)$ & 11.35 & -2.27 & 16. & 84 & $1.69(-6)$ & 8.48 & 2 \\
\hline $1433+538$ & GD 337 & 22,410 & ( 358$)$ & $7.80(0.05)$ & $0.53(0.02)$ & 10.23 & -1.28 & 16.09 & 148 & $3.06(-7)$ & 7.55 & 13 \\
\hline $1434+289$ & TON 210 & 32,800 & ( 499) & $8.00(0.06)$ & $0.65(0.03)$ & 9.74 & -0.73 & 15.80 & 163 & $1.47(-7)$ & 6.93 & \\
\hline $1436+526$ & & 23,440 & (436) & $7.94(0.06)$ & $0.60(0.03)$ & 10.36 & -1.29 & 16.43 & 163 & $3.54(-7)$ & 7.59 & 2 \\
\hline $1443+337$ & & 29,760 & $(455)$ & $7.83(0.05)$ & $0.56(0.03)$ & 9.67 & -0.79 & 16.39 & 220 & $1.41(-7)$ & 7.03 & 1 \\
\hline $1446+286$ & TON 214 & 22,890 & ( 348$)$ & $8.42(0.05)$ & $0.89(0.03)$ & 11.16 & -1.64 & 14.54 & 47 & $9.96(-7)$ & 8.10 & \\
\hline $1448+078$ & G66-32 & 14,170 & ( 228$)$ & $7.75(0.05)$ & $0.48(0.02)$ & 10.98 & -2.07 & 15.46 & 78 & $9.87(-7)$ & 8.29 & \\
\hline $1449+168$ & & 21,600 & $(336)$ & $7.88(0.05)$ & $0.56(0.02)$ & 10.41 & -1.39 & 15.44 & 101 & $3.88(-7)$ & 7.69 & \\
\hline $1451+006$ & GD 173 & 24,930 & $(380)$ & $7.89(0.05)$ & $0.58(0.02)$ & 10.15 & -1.14 & 15.20 & 102 & $2.67(-7)$ & 7.39 & 13 \\
\hline $1452-043$ & & 23,530 & $(472)$ & $8.19(0.06)$ & $0.74(0.04)$ & 10.73 & -1.44 & 16.22 & 125 & $5.67(-7)$ & 7.88 & \\
\hline $1456+298$ & G166-58 & 7390 & (106) & $7.97(0.05)$ & $0.58(0.03)$ & 13.28 & -3.34 & 15.60 & 29 & $2.82(-5)$ & 9.14 & \\
\hline $1457-086$ & & 21,450 & $(351)$ & $7.97(0.05)$ & $0.62(0.03)$ & 10.56 & -1.46 & 15.77 & 110 & $4.75(-7)$ & 7.80 & \\
\hline $1458+172$ & & 21,950 & $(471)$ & $7.43(0.06)$ & $0.41(0.02)$ & 9.62 & -1.06 & 16.3 & 216 & $1.46(-7)$ & 7.63 & 2 \\
\hline $1459+219$ & & 18,000 & ( 274) & $7.98(0.05)$ & $0.61(0.03)$ & 10.88 & -1.78 & 16. & 105 & $7.73(-7)$ & 8.10 & 1,2 \\
\hline $1459+347$ & & 21,520 & $(346)$ & $8.48(0.05)$ & $0.92(0.03)$ & 11.36 & -1.79 & 15.74 & 75 & $1.34(-6)$ & 8.23 & \\
\hline $1459+306$ & & 25,070 & ( 389$)$ & $7.97(0.05)$ & $0.62(0.03)$ & 10.26 & -1.18 & 13.98 & 55 & $3.05(-7)$ & 7.46 & 1 \\
\hline $1501+032$ & & 13,770 & $(362)$ & $7.88(0.06)$ & $0.54(0.03)$ & 11.21 & -2.19 & 15.48 & 71 & $1.38(-6)$ & 8.41 & \\
\hline
\end{tabular}


Table 1-Continued

\begin{tabular}{|c|c|c|c|c|c|c|c|c|c|c|c|c|}
\hline$P G$ & Name & $T_{\text {eff }}$ & $(\mathrm{K})$ & $\log g$ & $M / M_{\odot}$ & $M_{V}$ & $\log L / L_{\odot}$ & $V$ & $D(\mathrm{pc})$ & $1 / V_{\max }$ & $\log \tau$ & Notes \\
\hline $1502+351$ & & 18,120 & ( 275) & $8.13(0.05)$ & $0.70(0.03)$ & 11.09 & -1.86 & 15.78 & 86 & $1.01(-6)$ & 8.22 & \\
\hline $1507+021$ & & 19,580 & (433) & $7.87(0.07)$ & $0.56(0.04)$ & 10.57 & -1.57 & 16.49 & 152 & $4.99(-7)$ & 7.88 & \\
\hline $1507+220$ & & 19,340 & (312) & $7.91(0.05)$ & $0.57(0.03)$ & 10.65 & -1.61 & 15.05 & 75 & $5.55(-7)$ & 7.93 & 2 \\
\hline $1508+549$ & & 16,970 & $(265)$ & $7.86(0.05)$ & $0.54(0.02)$ & 10.82 & -1.82 & 15.61 & 90 & $7.27(-7)$ & 8.10 & \\
\hline $1508+637$ & GD 340 & 10,450 & ( 149$)$ & $8.12(0.05)$ & $0.68(0.03)$ & 12.18 & -2.82 & 14.65 & 31 & $5.50(-6)$ & 8.86 & \\
\hline $1509+323$ & GD 178 & 13,970 & $(259)$ & $7.98(0.05)$ & $0.60(0.03)$ & 11.34 & -2.23 & 14.11 & 35 & $1.62(-6)$ & 8.45 & \\
\hline $1511+009$ & LB 769 & 27,590 & ( 409) & $7.83(0.05)$ & $0.56(0.02)$ & 9.85 & -0.93 & 15.87 & 160 & $1.77(-7)$ & 7.16 & \\
\hline $1513+442$ & & 29,230 & (429) & $7.84(0.05)$ & $0.57(0.02)$ & 9.74 & -0.83 & 15.44 & 138 & $1.53(-7)$ & 7.06 & \\
\hline $1515+669$ & & 10,320 & ( 155$)$ & $8.40(0.06)$ & $0.86(0.04)$ & 12.68 & -3.02 & 15.33 & 33 & $1.07(-5)$ & 9.05 & 2 \\
\hline $1519+500$ & & 28,730 & $(526)$ & $7.40(0.07)$ & $0.42(0.02)$ & 9.01 & -0.55 & 16.45 & 307 & $6.63(-8)$ & 7.06 & \\
\hline $1519+384$ & & 19,620 & ( 348$)$ & $7.98(0.05)$ & $0.61(0.03)$ & 10.73 & -1.63 & 15.88 & 107 & $6.08(-7)$ & 7.97 & \\
\hline $1521+311$ & TON 229 & 25,630 & ( 386$)$ & $7.94(0.05)$ & $0.61(0.03)$ & 10.17 & -1.13 & 15.19 & 101 & $2.70(-7)$ & 7.38 & \\
\hline $1525+258$ & TON 235 & 22,290 & ( 351$)$ & $8.28(0.05)$ & $0.80(0.03)$ & 10.98 & -1.59 & 15.65 & 85 & $8.04(-7)$ & 8.04 & 2 \\
\hline $1526+014$ & & 50,420 & ( 1033 ) & $7.82(0.07)$ & $0.61(0.03)$ & 8.82 & +0.17 & 16.69 & 375 & $4.90(-8)$ & 6.34 & \\
\hline $1527+091$ & & 21,520 & $(344)$ & $8.02(0.05)$ & $0.64(0.03)$ & 10.63 & -1.49 & 14.29 & 53 & $5.17(-7)$ & 7.85 & \\
\hline $1531-023$ & GD 185 & 18,620 & $(285)$ & $8.41(0.05)$ & $0.88(0.03)$ & 11.49 & -1.99 & 14.00 & 31 & $1.71(-6)$ & 8.37 & \\
\hline $1532+034$ & & 66,330 & ( 2299) & $7.56(0.12)$ & $0.56(0.04)$ & 8.00 & +0.87 & 16.02 & 401 & $2.14(-8)$ & 5.95 & \\
\hline $1533-057$ & & 20,000 & (1039) & $8.50(0.30)$ & $0.94(0.18)$ & 11.53 & -1.93 & 15.33 & 57 & $1.73(-6)$ & 8.39 & 15 \\
\hline $1534+504$ & GD 347 & 8950 & $(128)$ & $8.07(0.05)$ & $0.64(0.03)$ & 12.68 & -3.06 & 15.71 & 40 & $1.11(-5)$ & 9.00 & \\
\hline $1535+293$ & TON 797 & 24,470 & ( 369$)$ & $7.92(0.05)$ & $0.60(0.02)$ & 10.24 & -1.20 & 16.07 & 146 & $3.01(-7)$ & 7.47 & \\
\hline $1537+652$ & GD 348 & 9740 & $(142)$ & $8.15(0.06)$ & $0.69(0.04)$ & 12.47 & -2.96 & 14.64 & 27 & $8.18(-6)$ & 8.96 & \\
\hline $1541+651$ & & 11,600 & ( 178$)$ & $8.10(0.05)$ & $0.67(0.03)$ & 11.85 & -2.63 & 15.81 & 61 & $3.46(-6)$ & 8.73 & 2,6 \\
\hline $1547+057$ & & 24,850 & $(373)$ & $8.41(0.05)$ & $0.88(0.03)$ & 10.98 & -1.49 & 15.92 & 97 & $7.67(-7)$ & 7.97 & \\
\hline $1547+016$ & & 76,910 & ( 3426$)$ & $7.57(0.15)$ & $0.59(0.04)$ & 7.85 & +1.14 & 15.95 & 417 & $1.85(-8)$ & 5.75 & \\
\hline $1548+406$ & & 54,530 & ( 1217 ) & $7.61(0.08)$ & $0.54(0.03)$ & 8.34 & +0.47 & 15.89 & 323 & $2.99(-8)$ & 6.24 & \\
\hline $1548+149$ & & 20,520 & ( 318$)$ & $7.89(0.05)$ & $0.57(0.02)$ & 10.53 & -1.50 & 15.11 & 82 & $4.59(-7)$ & 7.81 & \\
\hline $1550+183$ & GD 194 & 14,260 & ( 271) & $8.25(0.05)$ & $0.77(0.03)$ & 11.70 & -2.36 & 14.79 & 41 & $2.64(-6)$ & 8.59 & \\
\hline $1553+354$ & & 25,580 & $(382)$ & $7.83(0.05)$ & $0.55(0.02)$ & 10.01 & -1.06 & 14.75 & 88 & $2.22(-7)$ & 7.30 & \\
\hline $1554+215$ & & 26,270 & ( 398$)$ & $7.83(0.05)$ & $0.55(0.02)$ & 9.95 & -1.01 & 15.23 & 113 & $2.05(-7)$ & 7.25 & 2 \\
\hline $1554+262$ & & 21,220 & ( 353$)$ & $7.61(0.05)$ & $0.45(0.02)$ & 10.03 & -1.26 & 16.87 & 233 & $2.42(-7)$ & 7.73 & \\
\hline
\end{tabular}


Table 1-Continued

\begin{tabular}{|c|c|c|c|c|c|c|c|c|c|c|c|c|}
\hline$P G$ & Name & $T_{\text {eff }}$ & $(\mathrm{K})$ & $\log g$ & $M / M_{\odot}$ & $M_{V}$ & $\log L / L_{\odot}$ & V & $D(\mathrm{pc})$ & $1 / V_{\max }$ & $\log \tau$ & Notes \\
\hline $1559+129$ & & 29,220 & $(434)$ & $7.97(0.05)$ & $0.63(0.03)$ & 9.94 & -0.92 & 16.88 & 243 & $1.96(-7)$ & 7.13 & \\
\hline $1600+369$ & $\mathrm{R} 808$ & 11,160 & ( 161) & $8.04(0.05)$ & $0.63(0.03)$ & 11.86 & -2.66 & 14.36 & 31 & $3.55(-6)$ & 8.73 & 6 \\
\hline $1600+308$ & TON 252 & 58,060 & $(1741)$ & $7.38(0.11)$ & $0.49(0.03)$ & 7.84 & +0.75 & 15.37 & 321 & $1.86(-8)$ & 5.89 & 2 \\
\hline $1601+581$ & & 14,670 & $(238)$ & $7.84(0.05)$ & $0.53(0.02)$ & 11.05 & -2.06 & 14.3 & 44 & $1.07(-6)$ & 8.30 & 2 \\
\hline $1603+432$ & & 36,950 & ( 553$)$ & $7.91(0.05)$ & $0.62(0.02)$ & 9.39 & -0.46 & 14.86 & 124 & $9.53(-8)$ & 6.71 & \\
\hline $1605+684$ & & 21,090 & $(410)$ & $7.97(0.06)$ & $0.61(0.03)$ & 10.59 & -1.49 & 16.02 & 122 & $4.92(-7)$ & 7.83 & 2 \\
\hline $1608+419$ & & 16,680 & $(326)$ & $7.95(0.06)$ & $0.59(0.03)$ & 10.98 & -1.90 & 16.28 & 114 & $9.07(-7)$ & 8.20 & 2 \\
\hline $1608+119$ & & 20,650 & $(345)$ & $7.90(0.05)$ & $0.57(0.03)$ & 10.52 & -1.49 & 15.31 & 90 & $4.56(-7)$ & 7.80 & \\
\hline $1609+135$ & G138-8 & 9320 & ( 133$)$ & $8.64(0.05)$ & $1.01(0.03)$ & 13.48 & -3.37 & 15.095 & 21 & $3.21(-5)$ & 9.38 & \\
\hline $1609+045$ & & 29,120 & $(427)$ & $7.87(0.05)$ & $0.58(0.02)$ & 9.79 & -0.86 & 15.26 & 124 & $1.63(-7)$ & 7.07 & \\
\hline $1609+631$ & & 30,400 & $(446)$ & $8.67(0.05)$ & $1.05(0.03)$ & 11.05 & -1.33 & 16.68 & 133 & $7.62(-7)$ & 7.94 & \\
\hline $1610+167$ & GD 196 & 14,390 & $(240)$ & $7.84(0.05)$ & $0.52(0.02)$ & 11.07 & -2.09 & 15.67 & 83 & $1.12(-6)$ & 8.32 & \\
\hline $1614+137$ & & 22,430 & $(340)$ & $7.33(0.05)$ & $0.40(0.01)$ & 9.39 & -0.94 & 15.24 & 148 & $1.10(-7)$ & 7.48 & \\
\hline $1619+525$ & & 18,040 & ( 271) & $7.90(0.05)$ & $0.56(0.02)$ & 10.76 & -1.73 & 15.81 & 102 & $6.56(-7)$ & 8.03 & 2 \\
\hline $1619+123$ & & 16,460 & ( 251$)$ & $7.77(0.05)$ & $0.50(0.02)$ & 10.74 & -1.81 & 14.62 & 59 & $6.62(-7)$ & 8.08 & \\
\hline $1620+513$ & & 20,890 & $(382)$ & $7.92(0.05)$ & $0.58(0.03)$ & 10.53 & -1.48 & 15.97 & 122 & $4.60(-7)$ & 7.80 & \\
\hline $1620+648$ & & 30,280 & (438) & $7.86(0.05)$ & $0.58(0.02)$ & 9.69 & -0.78 & 15.88 & 172 & $1.43(-7)$ & 7.00 & 2 \\
\hline $1620+260$ & GD 200 & 28,290 & ( 417) & $7.78(0.05)$ & $0.54(0.02)$ & 9.72 & -0.85 & 15.53 & 145 & $1.51(-7)$ & 7.10 & \\
\hline $1622+324$ & & 68,280 & ( 3387$)$ & $7.56(0.17)$ & $0.57(0.05)$ & 7.97 & +0.93 & 16.38 & 481 & $2.07(-8)$ & 5.93 & 1 \\
\hline $1626+409$ & & 21,370 & $(418)$ & $8.02(0.06)$ & $0.64(0.03)$ & 10.64 & -1.50 & 16.7 & 163 & $5.23(-7)$ & 7.86 & \\
\hline $1632+177$ & & 10,100 & ( 143$)$ & $7.96(0.04)$ & $0.58(0.03)$ & 12.05 & -2.78 & 13.05 & 15 & $4.63(-6)$ & 8.79 & \\
\hline $1633+677$ & & 23,660 & ( 388$)$ & $7.97(0.05)$ & $0.62(0.03)$ & 10.37 & -1.29 & 16.25 & 149 & $3.60(-7)$ & 7.59 & 2 \\
\hline $1633+434$ & G180-63 & 6690 & ( 116$)$ & $8.20(0.14)$ & $0.72(0.09)$ & 14.01 & -3.65 & 14.82 & 14 & $8.50(-5)$ & 9.45 & \\
\hline $1635+608$ & & 25,750 & $(450)$ & $7.91(0.06)$ & $0.59(0.03)$ & 10.11 & -1.10 & 15.82 & 138 & $2.52(-7)$ & 7.34 & \\
\hline $1636+351$ & & 37,170 & $(564)$ & $7.97(0.05)$ & $0.65(0.03)$ & 9.48 & -0.49 & 15.95 & 197 & $1.06(-7)$ & 6.72 & \\
\hline $1637+335$ & G180-65 & 10,150 & ( 149) & $8.17(0.06)$ & $0.71(0.04)$ & 12.35 & -2.90 & 14.65 & 28 & $6.95(-6)$ & 8.92 & \\
\hline $1639+153$ & G138-56 & 7480 & ( 110$)$ & $8.42(0.06)$ & $0.87(0.04)$ & 13.91 & -3.59 & 15.66 & 22 & $6.53(-5)$ & 9.49 & \\
\hline $1640+458$ & LB 953 & 18,840 & ( 313$)$ & $7.82(0.05)$ & $0.53(0.03)$ & 10.58 & -1.61 & 16.31 & 140 & $5.09(-7)$ & 7.91 & 5 \\
\hline $1640+690$ & & 17,840 & ( 283) & $8.01(0.05)$ & $0.63(0.03)$ & 10.94 & -1.82 & 16.35 & 120 & $8.42(-7)$ & 8.14 & \\
\hline $1640+114$ & & 19,630 & ( 308$)$ & $8.03(0.05)$ & $0.64(0.03)$ & 10.80 & -1.65 & 16.03 & 111 & $6.68(-7)$ & 8.01 & \\
\hline
\end{tabular}


Table 1-Continued

\begin{tabular}{|c|c|c|c|c|c|c|c|c|c|c|c|c|}
\hline$P G$ & Name & $T_{\text {eff }}$ & $(\mathrm{K})$ & $\log g$ & $M / M_{\odot}$ & $M_{V}$ & $\log L / L_{\odot}$ & V & $D(\mathrm{pc})$ & $1 / V_{\max }$ & $\log \tau$ & Notes \\
\hline $1641+388$ & GD 357 & 15,570 & $(304)$ & $7.95(0.06)$ & $0.59(0.03)$ & 11.10 & -2.02 & 14.41 & 45 & $1.11(-6)$ & 8.29 & \\
\hline $1642+414$ & & 28,720 & $(454)$ & $8.24(0.06)$ & $0.79(0.03)$ & 10.40 & -1.12 & 16.21 & 145 & $3.45(-7)$ & 7.51 & 5 \\
\hline $1642+386$ & & 45,930 & $(866)$ & $7.64(0.07)$ & $0.53(0.02)$ & 8.62 & +0.12 & 16.58 & 390 & $4.02(-8)$ & 6.46 & \\
\hline $1643+144$ & & 26,850 & $(512)$ & $7.91(0.07)$ & $0.60(0.04)$ & 10.03 & -1.03 & 15.90 & 149 & $2.24(-7)$ & 7.26 & 1 \\
\hline $1646+062$ & & 29,940 & $(457)$ & $7.98(0.05)$ & $0.64(0.03)$ & 9.90 & -0.88 & 16.03 & 168 & $1.84(-7)$ & 7.09 & 1 \\
\hline $1647+376$ & & 21,980 & $(340)$ & $7.89(0.05)$ & $0.57(0.02)$ & 10.39 & -1.37 & 15.00 & 83 & $3.77(-7)$ & 7.66 & \\
\hline $1648+371$ & & 43,110 & (918) & $7.56(0.08)$ & $0.49(0.03)$ & 8.56 & +0.06 & 15.87 & 289 & $3.82(-8)$ & 6.49 & \\
\hline $1654+637$ & GD 515 & 15,070 & ( 299) & $7.63(0.06)$ & $0.44(0.03)$ & 10.67 & -1.88 & 15.65 & 98 & $6.37(-7)$ & 8.31 & \\
\hline $1655+215$ & G169-34 & 9310 & ( 133$)$ & $8.20(0.05)$ & $0.73(0.03)$ & 12.73 & -3.07 & 14.10 & 18 & $1.16(-5)$ & 9.04 & \\
\hline $1657+344$ & & 52,240 & ( 1274$)$ & $7.64(0.09)$ & $0.55(0.03)$ & 8.46 & +0.36 & 16.42 & 390 & $3.39(-8)$ & 6.32 & 5 \\
\hline $1658+441$ & & 30,510 & $(598)$ & $9.36(0.09)$ & $1.31(0.05)$ & 12.49 & -1.91 & 14.62 & 26 & $5.00(-6)$ & 8.58 & 16 \\
\hline $1659+303$ & & 13,600 & $(310)$ & $7.95(0.05)$ & $0.58(0.03)$ & 11.35 & -2.26 & 14.99 & 53 & $1.66(-6)$ & 8.47 & 17 \\
\hline $1707+476$ & & 26,350 & ( 403) & $7.80(0.05)$ & $0.54(0.02)$ & 9.90 & -0.99 & 15.16 & 112 & $1.92(-7)$ & 7.23 & \\
\hline $1713+333$ & GD 360 & 22,120 & $(331)$ & $7.43(0.05)$ & $0.41(0.01)$ & 9.60 & -1.04 & 14.49 & 95 & $1.42(-7)$ & 7.61 & 13 \\
\hline $1720+361$ & & 13,670 & $(281)$ & $7.83(0.05)$ & $0.52(0.03)$ & 11.16 & -2.18 & 15.34 & 68 & $1.29(-6)$ & 8.39 & 2 \\
\hline $1725+587$ & LB 335 & 55,800 & ( 1189) & $8.28(0.07)$ & $0.85(0.04)$ & 9.51 & +0.03 & 15.45 & 153 & $1.05(-7)$ & 6.14 & \\
\hline $2056+033$ & & 48,400 & (1310) & $7.78(0.10)$ & $0.59(0.04)$ & 8.80 & +0.12 & 16.26 & 310 & $4.82(-8)$ & 6.40 & \\
\hline $2115+011$ & & 25,220 & $(445)$ & $7.83(0.06)$ & $0.55(0.03)$ & 10.04 & -1.09 & 15.5 & 123 & $2.31(-7)$ & 7.33 & \\
\hline $2120+055$ & & 35,860 & $(542)$ & $7.80(0.05)$ & $0.57(0.02)$ & 9.25 & -0.44 & 16.3 & 257 & $8.20(-8)$ & 6.75 & \\
\hline $2150+021$ & & 41,320 & (668) & $7.72(0.06)$ & $0.55(0.02)$ & 8.91 & -0.13 & 16.40 & 315 & $5.50(-8)$ & 6.58 & \\
\hline $2204+071$ & & 24,790 & $(383)$ & $7.95(0.05)$ & $0.61(0.03)$ & 10.26 & -1.19 & 15.86 & 132 & $3.05(-7)$ & 7.47 & \\
\hline $2207+142$ & G18-34 & 7620 & $(112)$ & $8.21(0.07)$ & $0.73(0.05)$ & 13.52 & -3.43 & 15.62 & 26 & $3.76(-5)$ & 9.28 & \\
\hline $2220+134$ & & 22,680 & $(344)$ & $8.41(0.05)$ & $0.88(0.03)$ & 11.16 & -1.65 & 15.60 & 77 & $1.01(-6)$ & 8.11 & \\
\hline $2226+061$ & GD 236 & 15,280 & $(235)$ & $7.62(0.05)$ & $0.44(0.02)$ & 10.63 & -1.85 & 14.71 & 65 & $5.93(-7)$ & 8.28 & \\
\hline $2235+082$ & & 35,540 & $(554)$ & $7.98(0.06)$ & $0.65(0.03)$ & 9.56 & -0.57 & 15.42 & 148 & $1.17(-7)$ & 6.79 & 4 \\
\hline $2239+082$ & & 23,070 & $(438)$ & $7.86(0.06)$ & $0.56(0.03)$ & 10.27 & -1.27 & 16.58 & 182 & $3.18(-7)$ & 7.55 & \\
\hline $2244+031$ & & 59,230 & ( 1114) & $7.70(0.06)$ & $0.58(0.02)$ & 8.41 & +0.56 & 16.41 & 398 & $3.18(-8)$ & 6.15 & 1 \\
\hline $2246+223$ & G67-23 & 10,650 & ( 153$)$ & $8.80(0.05)$ & $1.10(0.02)$ & 13.32 & -3.26 & 14.35 & 16 & $2.51(-5)$ & 9.34 & \\
\hline $2257+138$ & & 27,560 & ( 403) & $8.33(0.05)$ & $0.84(0.03)$ & 10.65 & -1.25 & 16.65 & 158 & $4.77(-7)$ & 7.72 & \\
\hline $2257+162$ & & 24,580 & $(382)$ & $7.49(0.05)$ & $0.43(0.02)$ & 9.50 & -0.89 & 16.14 & 212 & $1.23(-7)$ & 7.23 & \\
\hline
\end{tabular}


Table 1-Continued

\begin{tabular}{|c|c|c|c|c|c|c|c|c|c|c|c|c|}
\hline$P G$ & Name & $T_{\text {eff }}$ & $(\mathrm{K})$ & $\log g$ & $M / M_{\odot}$ & $M_{V}$ & $\log L / L_{\odot}$ & $V$ & $D(\mathrm{pc})$ & $1 / V_{\max }$ & $\log \tau$ & Notes \\
\hline $2303+017$ & PHL 400 & 41,720 & ( 1007$)$ & $7.62(0.10)$ & $0.51(0.04)$ & 8.72 & -0.04 & 16.11 & 300 & $4.52(-8)$ & 6.57 & 2 \\
\hline $2303+243$ & & 11,480 & $(175)$ & $8.09(0.05)$ & $0.66(0.03)$ & 11.86 & -2.64 & 15.5 & 53 & $3.53(-6)$ & 8.74 & 6 \\
\hline $2306+131$ & & 13,250 & ( 283$)$ & $7.92(0.05)$ & $0.56(0.03)$ & 11.34 & -2.29 & 15.38 & 64 & $1.68(-6)$ & 8.47 & \\
\hline $2306+125$ & & 20,220 & ( 318$)$ & $8.05(0.05)$ & $0.66(0.03)$ & 10.79 & -1.62 & 15.23 & 77 & $6.51(-7)$ & 7.99 & \\
\hline $2308+051$ & PB 5280 & 35,950 & $(562)$ & $7.58(0.06)$ & $0.48(0.02)$ & 8.88 & -0.28 & 16.02 & 267 & $5.48(-8)$ & 6.72 & \\
\hline $2322+207$ & & 13,060 & $(260)$ & $7.84(0.05)$ & $0.52(0.03)$ & 11.26 & -2.27 & 15.59 & 73 & $1.52(-6)$ & 8.45 & \\
\hline $2324+060$ & PB 5379 & 15,750 & $(239)$ & $7.90(0.05)$ & $0.56(0.02)$ & 11.01 & -1.97 & 15.38 & 74 & $9.71(-7)$ & 8.24 & \\
\hline $2326+049$ & G29-38 & 11,820 & ( 175$)$ & $8.15(0.05)$ & $0.70(0.03)$ & 11.87 & -2.62 & 13.03 & 17 & $3.55(-6)$ & 8.74 & 6 \\
\hline $2328+108$ & & 21,910 & $(356)$ & $7.84(0.05)$ & $0.55(0.02)$ & 10.32 & -1.34 & 15.54 & 110 & $3.47(-7)$ & 7.62 & \\
\hline $2329+267$ & G128-72 & 11,730 & $(221)$ & $8.98(0.06)$ & $1.18(0.03)$ & 13.39 & -3.23 & 15.29 & 24 & $2.70(-5)$ & 9.31 & \\
\hline $2331+290$ & GD 251 & 27,320 & $(442)$ & $7.48(0.05)$ & $0.44(0.02)$ & 9.27 & -0.70 & 15.85 & 206 & $9.05(-8)$ & 6.96 & 7 \\
\hline $2336+063$ & PB 5486 & 16,520 & ( 260$)$ & $8.03(0.05)$ & $0.63(0.03)$ & 11.11 & -1.96 & 15.60 & 79 & $1.08(-6)$ & 8.27 & \\
\hline $2341+323$ & G130-5 & 12,570 & ( 186$)$ & $7.93(0.05)$ & $0.57(0.03)$ & 11.45 & -2.38 & 12.93 & 19 & $1.99(-6)$ & 8.53 & 1 \\
\hline $2345+305$ & & 29,060 & (495) & $7.72(0.07)$ & $0.51(0.03)$ & 9.56 & -0.76 & 16.41 & 234 & $1.23(-7)$ & 7.06 & \\
\hline $2349+286$ & & 37,720 & ( 589$)$ & $7.95(0.05)$ & $0.64(0.03)$ & 9.42 & -0.44 & 16.26 & 233 & $9.85(-8)$ & 6.69 & \\
\hline $2353+027$ & PB 5617 & 60,970 & ( 1495$)$ & $7.60(0.08)$ & $0.56(0.03)$ & 8.19 & +0.68 & 15.83 & 336 & $2.58(-8)$ & 6.08 & \\
\hline $2357+297$ & & 51,960 & ( 1070$)$ & $7.52(0.07)$ & $0.51(0.02)$ & 8.25 & +0.44 & 15.1 & 234 & $2.74(-8)$ & 6.24 & \\
\hline
\end{tabular}

Note. - (1) Binary; (2) $V$ magnitude derived from $B_{\mathrm{ph}}$; (3) DAO star, $T_{\text {eff }}$ and $\log g$ from Bergeron et al. 1994; (4) Misclassified in the PG catalog; (5) $V$ magnitude derived from $g$ and $r$ Sloan Digital Sky Survey; (6) ZZ Ceti star; (7) Double degenerate; (8) DA + magnetic DB, $T_{\text {eff }}$ and $\log g$ from Liebert et al. 1993; (9) Planetary nebula nucleus; (10) Magnetic, $T_{\text {eff }}$ and $\log g$ from Schmidt et al. 1986; (11) Magnetic, $T_{\text {eff }}$ and $\log g$ from Bergeron \& Liebert 2002; (12) Magnetic, $T_{\text {eff }}$ and $\log g$ from Liebert et al. 2003; (13) Double degenerate?; (14) Magnetic; (15) Magnetic, $T_{\text {eff }}$ and $\log g$ from Liebert et al. 1985; (16) Magnetic, $T_{\text {eff }}$ and $\log g$ from Schmidt et al. 1992; (17) Not in McCook \& Sion 1999. 
Table 2. Atmospheric Parameters of other DA Stars from the PG Survey

\begin{tabular}{|c|c|c|c|c|c|c|c|c|c|}
\hline$P G$ & Name & $T_{\text {eff }}$ & $(\mathrm{K})$ & $\log g$ & $M / M_{\odot}$ & $M_{V}$ & $\log L / L_{\odot}$ & $\log \tau$ & Notes \\
\hline $0019+150$ & PHL 802 & 30,800 & $(475)$ & $8.10(0.06)$ & $0.71(0.03)$ & 10.03 & -0.90 & 7.14 & \\
\hline $0101+059$ & PB 6250 & 14,190 & ( 394$)$ & $8.35(0.05)$ & $0.83(0.03)$ & 11.86 & -2.43 & 8.66 & \\
\hline $0232+035$ & Feige 24 & 65,290 & $(1553)$ & $7.24(0.08)$ & $0.47(0.02)$ & 7.39 & +1.09 & 5.40 & \\
\hline $0302+028$ & GD 41 & 35,230 & $(528)$ & $7.78(0.05)$ & $0.55(0.02)$ & 9.24 & -0.45 & 6.78 & 1 \\
\hline $0307+149$ & & 21,320 & $(347)$ & $8.03(0.05)$ & $0.64(0.03)$ & 10.66 & -1.51 & 7.87 & \\
\hline $0308+096$ & & 25,900 & $(451)$ & $8.08(0.06)$ & $0.68(0.03)$ & 10.37 & -1.20 & 7.54 & \\
\hline $0308+188$ & & 17,860 & $(264)$ & $7.85(0.04)$ & $0.54(0.02)$ & 10.71 & -1.72 & 8.01 & \\
\hline $0317+196$ & & 17,060 & $(260)$ & $7.90(0.05)$ & $0.56(0.02)$ & 10.86 & -1.83 & 8.12 & \\
\hline $0836+237$ & & 54,260 & $(1177)$ & $7.67(0.08)$ & $0.56(0.03)$ & 8.47 & +0.41 & 6.26 & \\
\hline $0839+232$ & & 25,030 & $(370)$ & $7.84(0.05)$ & $0.56(0.02)$ & 10.07 & -1.11 & 7.35 & \\
\hline $0901+598$ & & 50,570 & ( 1536$)$ & $7.63(0.11)$ & $0.54(0.04)$ & 8.48 & +0.31 & 6.35 & \\
\hline $0904+391$ & & 25,460 & $(386)$ & $8.07(0.05)$ & $0.68(0.03)$ & 10.39 & -1.22 & 7.56 & \\
\hline $0909+272$ & TON 393 & 48,060 & ( 1651) & $8.00(0.13)$ & $0.69(0.07)$ & 9.19 & -0.04 & 6.41 & \\
\hline $0920+375$ & & 18,340 & $(304)$ & $8.40(0.05)$ & $0.87(0.03)$ & 11.50 & -2.01 & 8.38 & \\
\hline $1053-092$ & & 23,080 & $(401)$ & $7.69(0.05)$ & $0.48(0.02)$ & 10.00 & -1.16 & 7.43 & 2 \\
\hline $1121+216$ & Ross 627 & 7470 & $(112)$ & $8.20(0.08)$ & $0.72(0.05)$ & 13.57 & -3.45 & 9.29 & \\
\hline $1123+189$ & & 51,680 & $(930)$ & $7.86(0.06)$ & $0.63(0.03)$ & 8.86 & +0.19 & 6.28 & \\
\hline $1231+465$ & TON 82 & 22,550 & ( 355$)$ & $7.94(0.05)$ & $0.60(0.03)$ & 10.42 & -1.35 & 7.66 & \\
\hline $1232+238$ & & 46,090 & $(1206)$ & $7.66(0.10)$ & $0.54(0.04)$ & 8.64 & +0.12 & 6.46 & \\
\hline $1300-099$ & & 14,920 & ( 441) & $8.16(0.07)$ & $0.71(0.04)$ & 11.49 & -2.22 & 8.51 & \\
\hline $1333+511$ & & 36,110 & ( 649) & $7.90(0.08)$ & $0.61(0.04)$ & 9.40 & -0.49 & 6.75 & \\
\hline $1413+015$ & & 50,050 & $(1345)$ & $7.72(0.10)$ & $0.57(0.04)$ & 8.65 & +0.23 & 6.37 & \\
\hline $1428+374$ & & 14,010 & $(221)$ & $7.36(0.05)$ & $0.36(0.01)$ & 10.35 & -1.83 & 8.34 & \\
\hline $1439+305$ & & 21,870 & $(416)$ & $7.96(0.06)$ & $0.61(0.03)$ & 10.51 & -1.42 & 7.76 & \\
\hline $1454+173$ & & 30,660 & $(481)$ & $8.14(0.06)$ & $0.73(0.04)$ & 10.10 & -0.94 & 7.20 & \\
\hline $1520+447$ & & 16,550 & $(297)$ & $7.89(0.05)$ & $0.56(0.03)$ & 10.89 & -1.87 & 8.16 & \\
\hline $1539-035$ & GD 189 & 10,080 & ( 148$)$ & $8.30(0.06)$ & $0.79(0.04)$ & 12.59 & -2.99 & 9.01 & \\
\hline $1614+160$ & & 17,000 & $(268)$ & $7.92(0.05)$ & $0.57(0.03)$ & 10.90 & -1.85 & 8.14 & \\
\hline $2309+105$ & GD 246 & 54,410 & $(964)$ & $7.90(0.06)$ & $0.65(0.03)$ & 8.88 & +0.25 & 6.23 & \\
\hline $2314+064$ & PB 5312 & 17,570 & ( 273) & $7.98(0.05)$ & $0.61(0.03)$ & 10.93 & -1.83 & 8.14 & \\
\hline
\end{tabular}

Note. - (1) Classified sd in the PG catalog; (2) Classified DB in the PG catalog. 
Table 3. White Dwarf Evolution Rates

\begin{tabular}{lccll}
\hline \hline $\log \tau$ & Delta $\tau$ & Main & Low & \multicolumn{1}{c}{ High } \\
\hline 6.25 & $2.16(6)$ & $4.21(-13)$ & 0.00 & $4.83(-14)$ \\
6.75 & $6.84(6)$ & $4.43(-13)$ & $2.75(-14)$ & 0.00 \\
7.25 & $2.16(7)$ & $5.06(-13)$ & $4.72(-14)$ & 0.00 \\
7.75 & $6.84(7)$ & $3.95(-13)$ & $1.66(-14)$ & $7.79(-14)$ \\
8.25 & $2.16(8)$ & $3.28(-13)$ & $1.01(-14)$ & $7.67(-14)$ \\
8.75 & $6.84(8)$ & $1.72(-13)$ & $3.31(-15)$ & $3.72(-14)$ \\
9.25 & $2.16(9)$ & $1.45(-13)$ & 0.00 & $1.41(-13)$ \\
\hline
\end{tabular}


Fig. 1.- Distribution of the signal-to-noise ratio for the 345 optical spectra secured for the analysis of the complete PG sample.

Fig. 2.- Optical spectra for a subsample of DA stars from the PG catalog. The spectra are normalized at $4500 \AA$, and are shifted vertically; the various zero points are indicated by long tick marks. The effective temperature decreases from upper left to bottom right.

Fig. 3.- Corrections that must be applied to transform atmospheric parameters obtained from LTE model spectra to account for NLTE effects. The differences are magnified three times in the $\log g$ direction. LTE models tend to overestimate both $T_{\text {eff }}$ and $\log g$.

Fig. 4.- Examples of the procedures used to define the continuum with pseudo-Gaussian profiles (top panel; $T_{\text {eff }} \sim 12,000 \mathrm{~K}$ ) or model spectra (bottom panel; $T_{\text {eff }} \sim 35,000 \mathrm{~K}$ ). See text for details. The dotted lines indicate the wavelength range used to define the continuum for each line.

Fig. 5.- Model fits to the individual Balmer line profiles of sample DA stars taken from Figure 2 in order of decreasing effective temperature. The lines range from $\mathrm{H} \beta$ (bottom) to $\mathrm{H} 8($ top $)$, each offset vertically by a factor of 0.2 .

Fig. 6. - $T_{\text {eff }}$ and $\log g$ values for all DA stars from the complete PG sample. The solid lines represent the carbon-core evolutionary models of Wood (1995) with thick hydrogen layers; numbers on the right hand side of the figure indicate the mass of each model in solar masses. The dotted lines represent the helium-core models of Althaus et al. (2001) for, from top to bottom, $M=0.196,0.242,0.292,0.360$, and $0.406 M_{\odot}$.

Fig. 7.- Comparison of atmospheric parameter solutions obtained under the assumption of LTE (open circles) and NLTE (filled circles).

Fig. 8.- Distribution of standard deviations in $T_{\text {eff }}$ and $\log g$ for individual white dwarf stars with multiple measurements, as a function of effective temperature. Standard deviations in $T_{\text {eff }}$ are expressed in percentage with respect to the average temperature of the star. The dotted lines represent the average standard deviations.

Fig. 9.- Comparison of our $T_{\text {eff }}$ and $\log g$ determinations of PG stars in common with the data sets of Finley et al. (1997), Vennes et al. (1997b), Marsh et al. (1997), Homeier et al. (1998), and Koester et al. (2001). In each panel we plot the differences between these investigations (others) and our results $(\mathrm{PG})$ as a function of our determinations of $T_{\text {eff }}$ or $\log g$. Effective temperatures are in units of $10^{3} \mathrm{~K}$.

Fig. 10.- Luminosity function of all DA stars in the complete PG sample (solid line) 
presented in half-magnitude bins, assuming a scale height for the Galaxy of $z_{0}=250 \mathrm{pc}$. The dotted line represents the results of Fleming et al. (1986), and the dashed line that of Darling (1994), shown here for comparison.

Fig. 11. - Masses of all DA stars in the complete PG sample as a function of $\log T_{\text {eff }}$, together with the theoretical isochrones from Wood (1995, solid lines) and Althaus et al. (2001, dotted lines). Isochrones are labeled in units of $\log \tau$, where $\tau$ is the white dwarf cooling age in years.

Fig. 12.- Surface gravity and mass distributions for the 297 DA stars in the complete PG sample with $T_{\text {eff }}>13,000 \mathrm{~K}$. The masses of DA stars below this value may be biased, as explained in the text. Mean values and standard deviations of both distributions are indicated in each panel.

Fig. 13. - The $1 / V_{\max }$ weighted mass distribution of the $297 \mathrm{PG}$ DA stars with $T_{\text {eff }}>13,000 \mathrm{~K}$.

Fig. 14. - The luminosity functions of the three arbitrary mass components as discussed in the text, with symbols as shown in the figure.

Fig. 15.- Absolute visual magnitudes $M_{V}$ as a function of $\log T_{\text {eff }}$ for all DA stars in the complete PG sample. The theoretical isochrones from Wood (1995, solid lines) and Althaus et al. (2001, dotted lines) are also shown, with corresponding masses indicated in the Figure. Isochrones are labeled in units of $\log \tau$, where $\tau$ is the white dwarf cooling age in years.

Fig. 16. - The number density of white dwarf weighted by $1 / V_{\max }$ is plotted for 0.5 dex intervals of the log of the cooling time, for the three mass components shown in

Fig. 17. - The spectrum published in Bergeron et al. (1994) (bottom) is displayed with a new, higher signal-to-noise ratio spectrum (top). Both appear to show a weak line due to He I $4471 \AA$. No other He I or He II features appear.

Fig. 18. - The 27 white dwarfs found in the EUVE and ROSAT all-sky surveys with mass estimates $>0.8 M_{\odot}$ are shown as "star" symbols, plotted in Galactic coordinates. Also shown as small dots are the positions of all $\mathrm{O}$ and $\mathrm{B}$ stars in the on-line Yale Bright Star Catalog. 


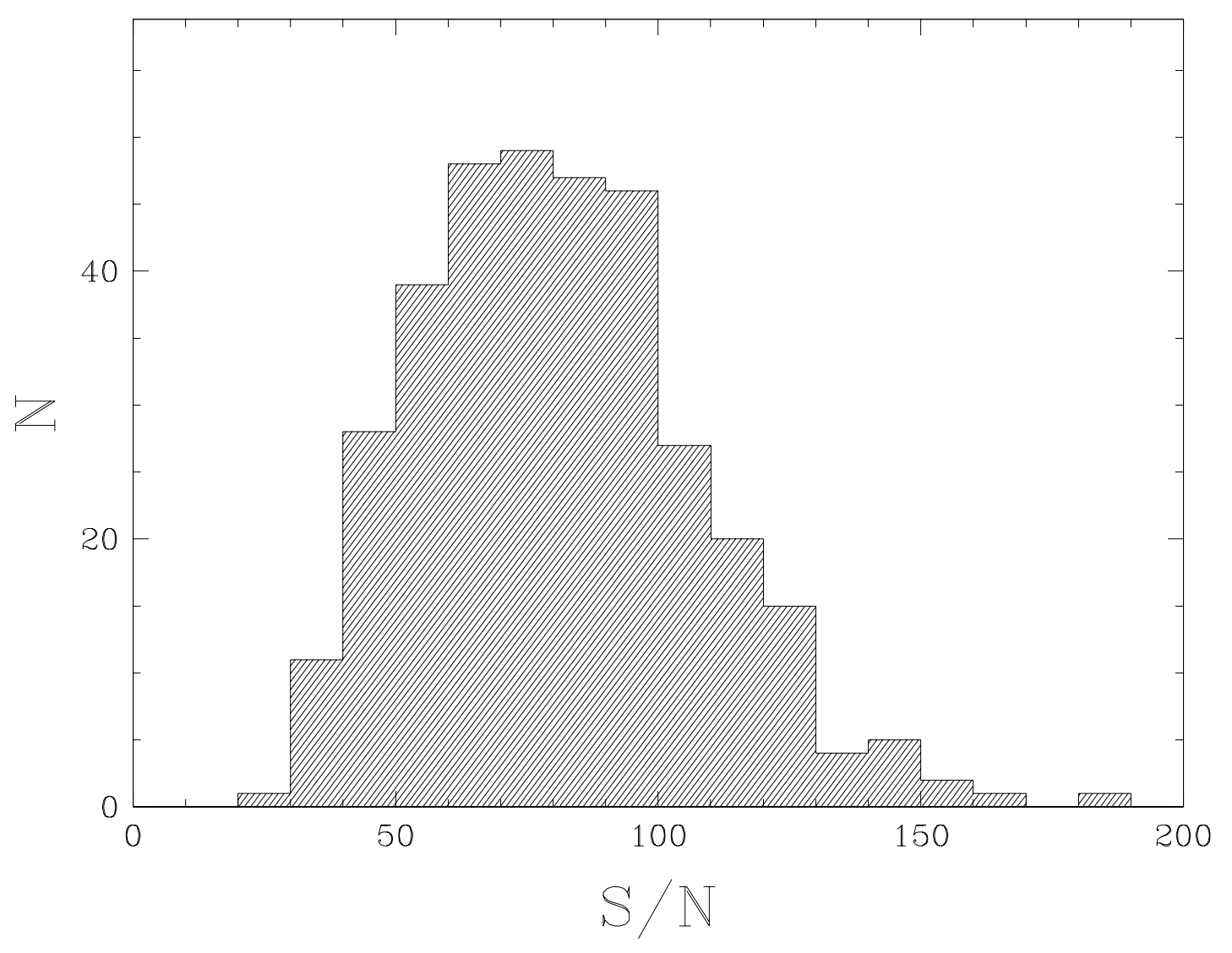

Figure 1 


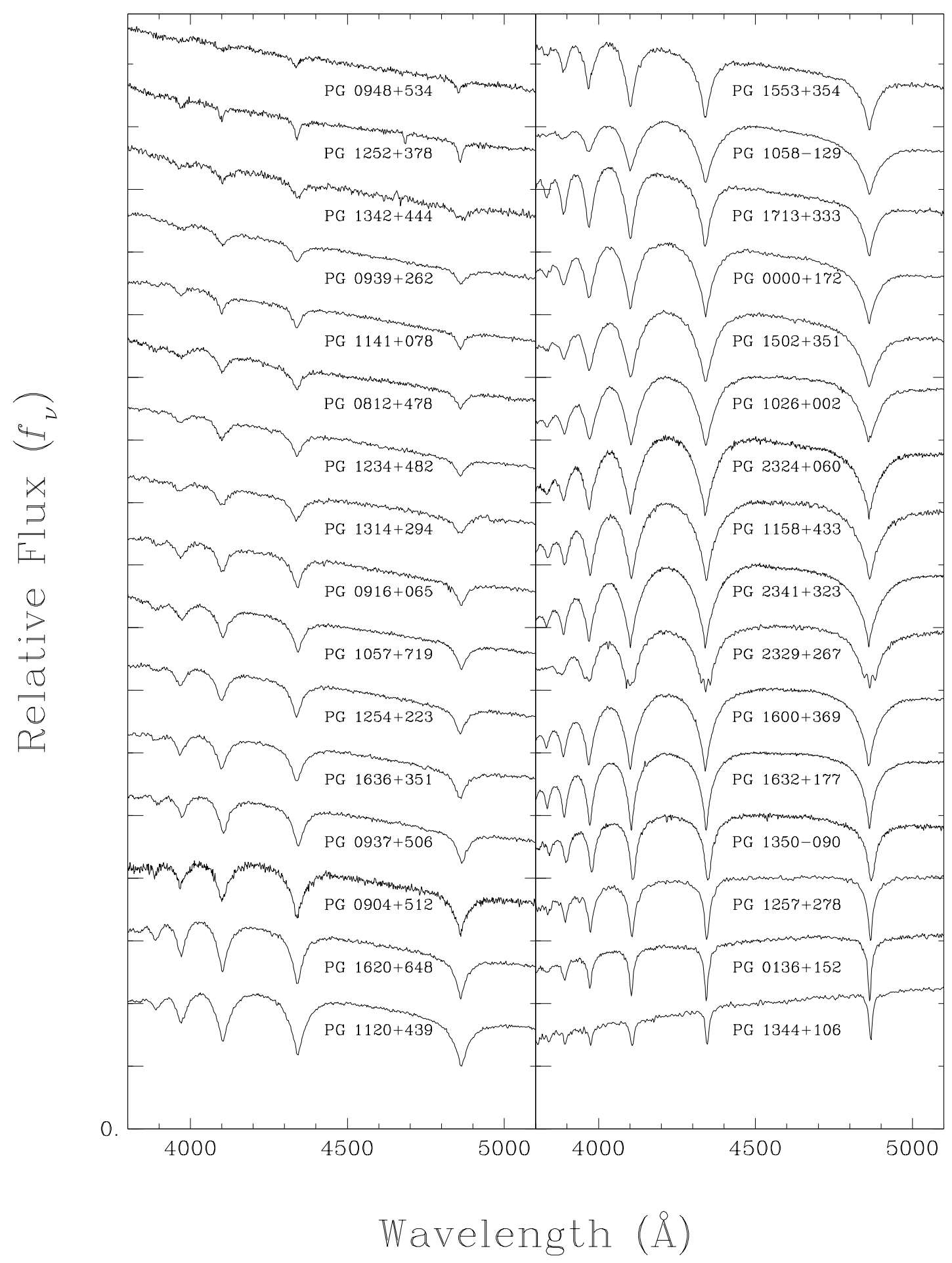

Figure 2 


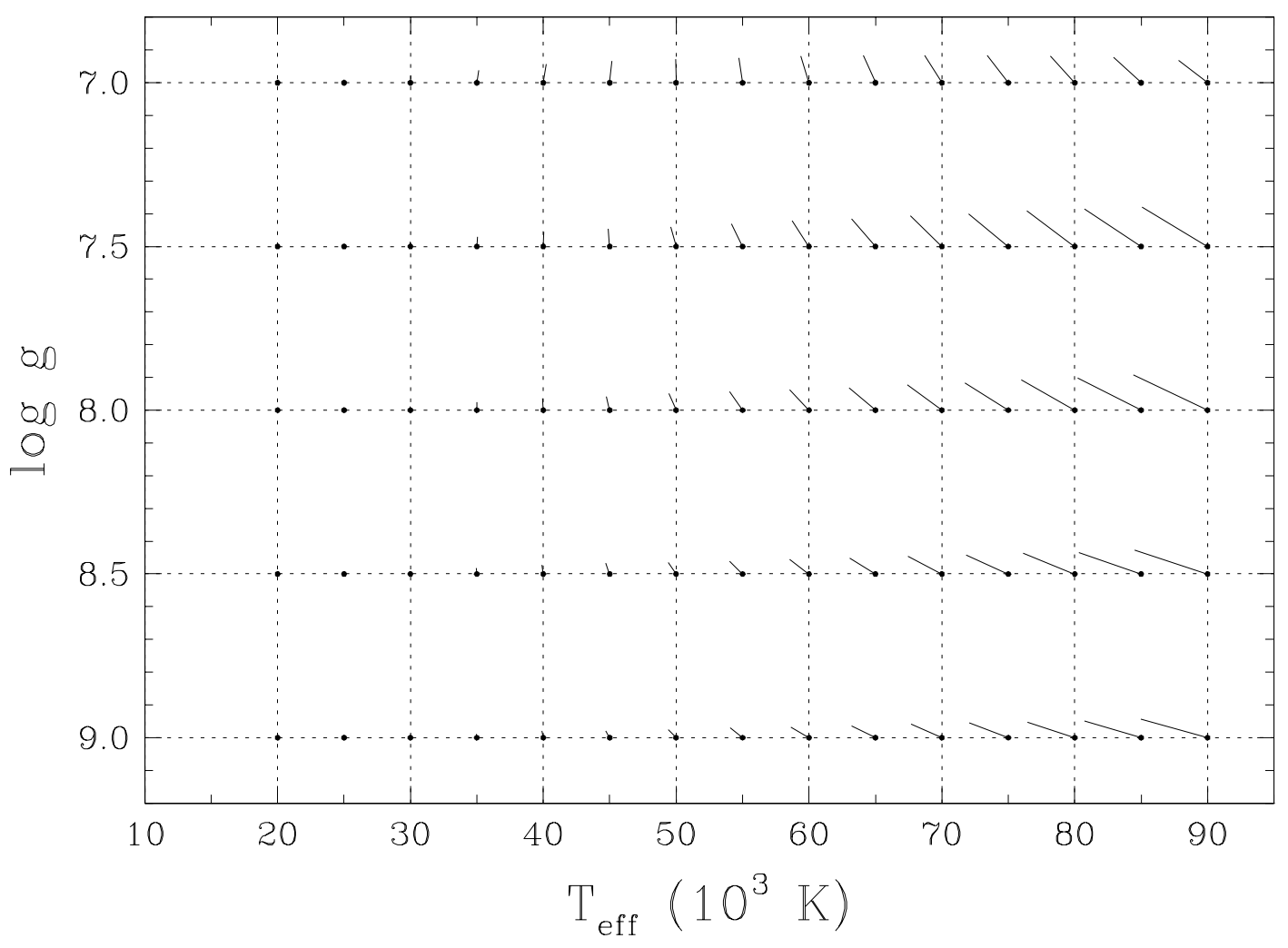

Figure 3 


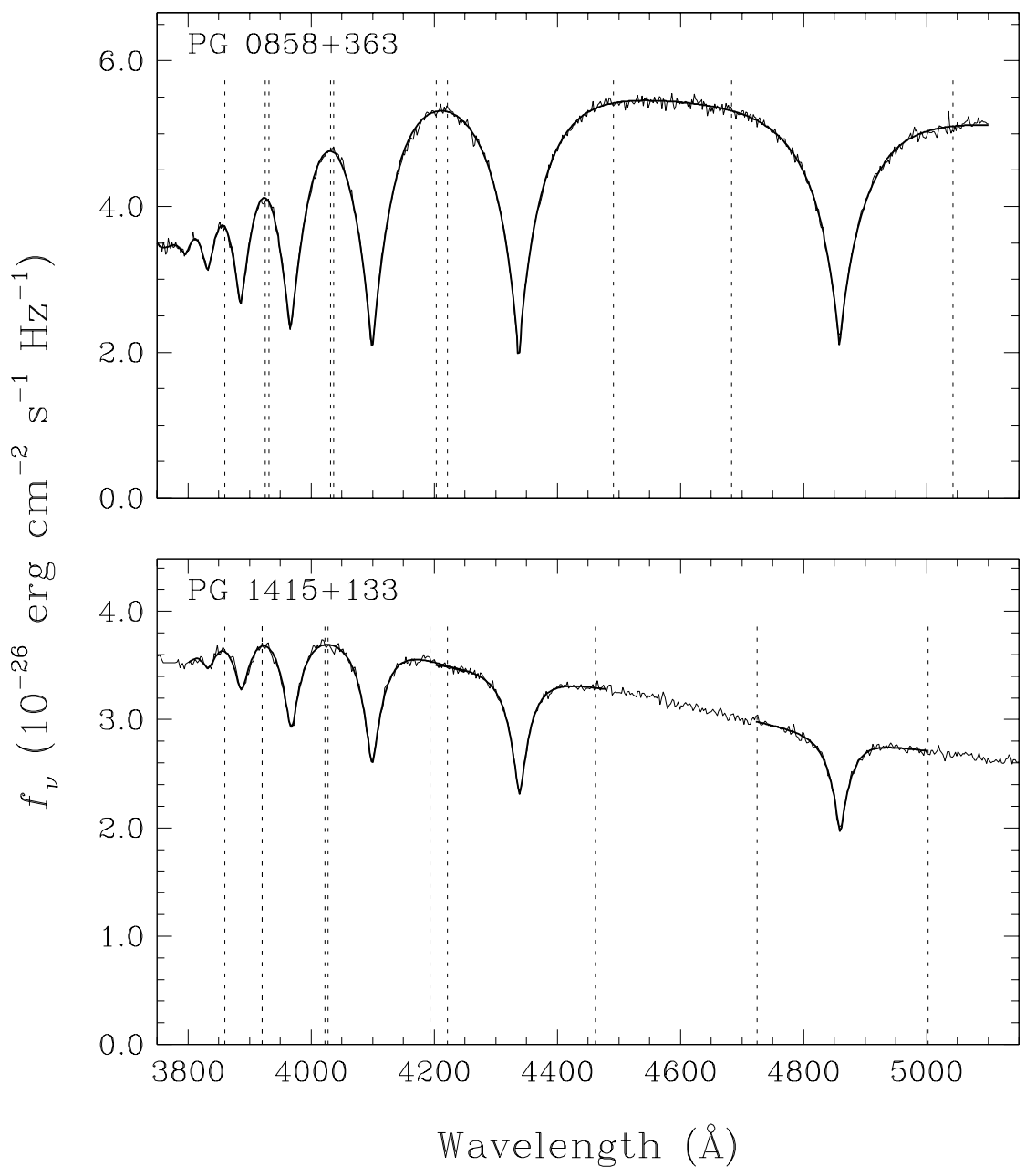

Figure 4 


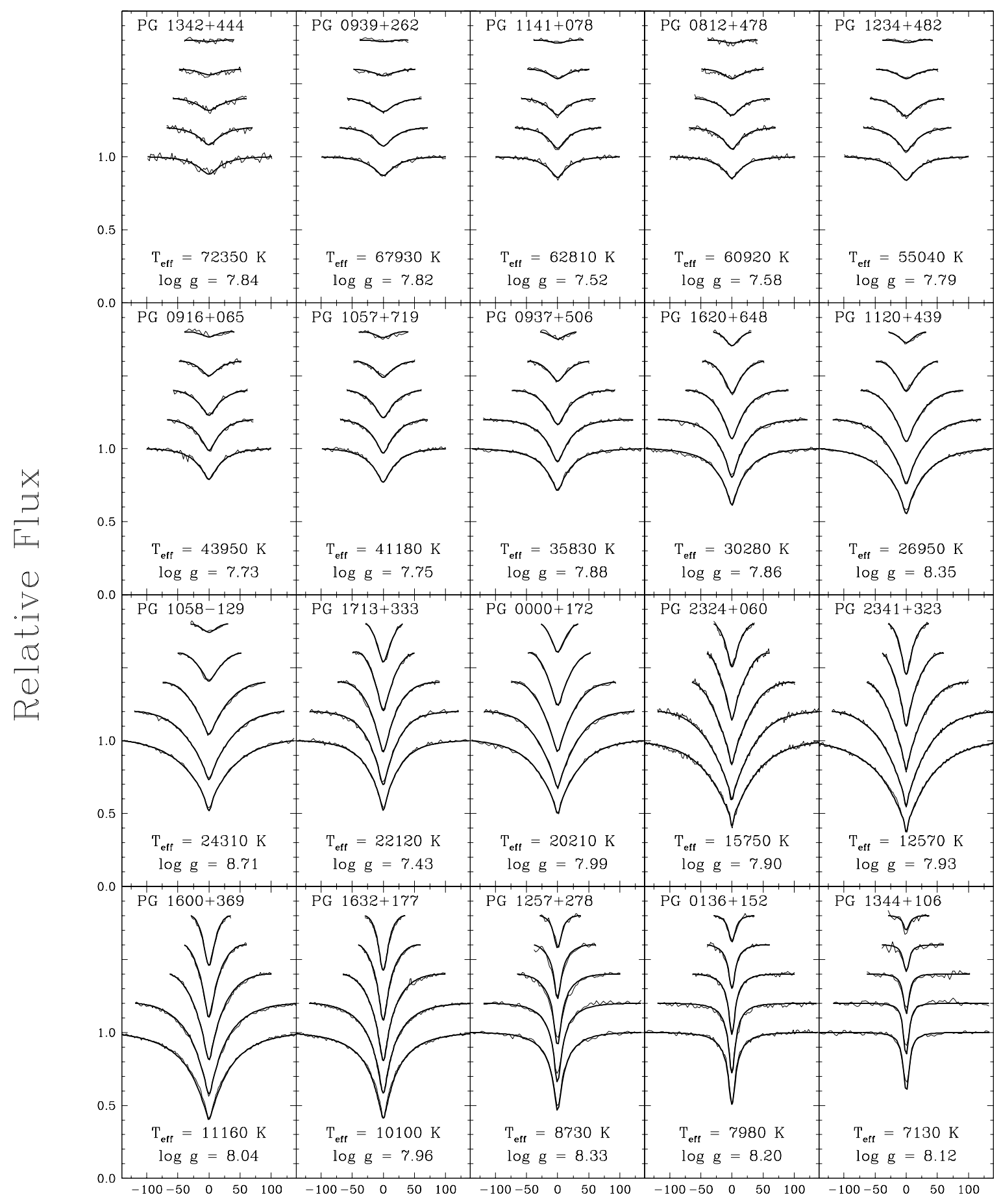

$\Delta \lambda(\AA)$

Figure 5 


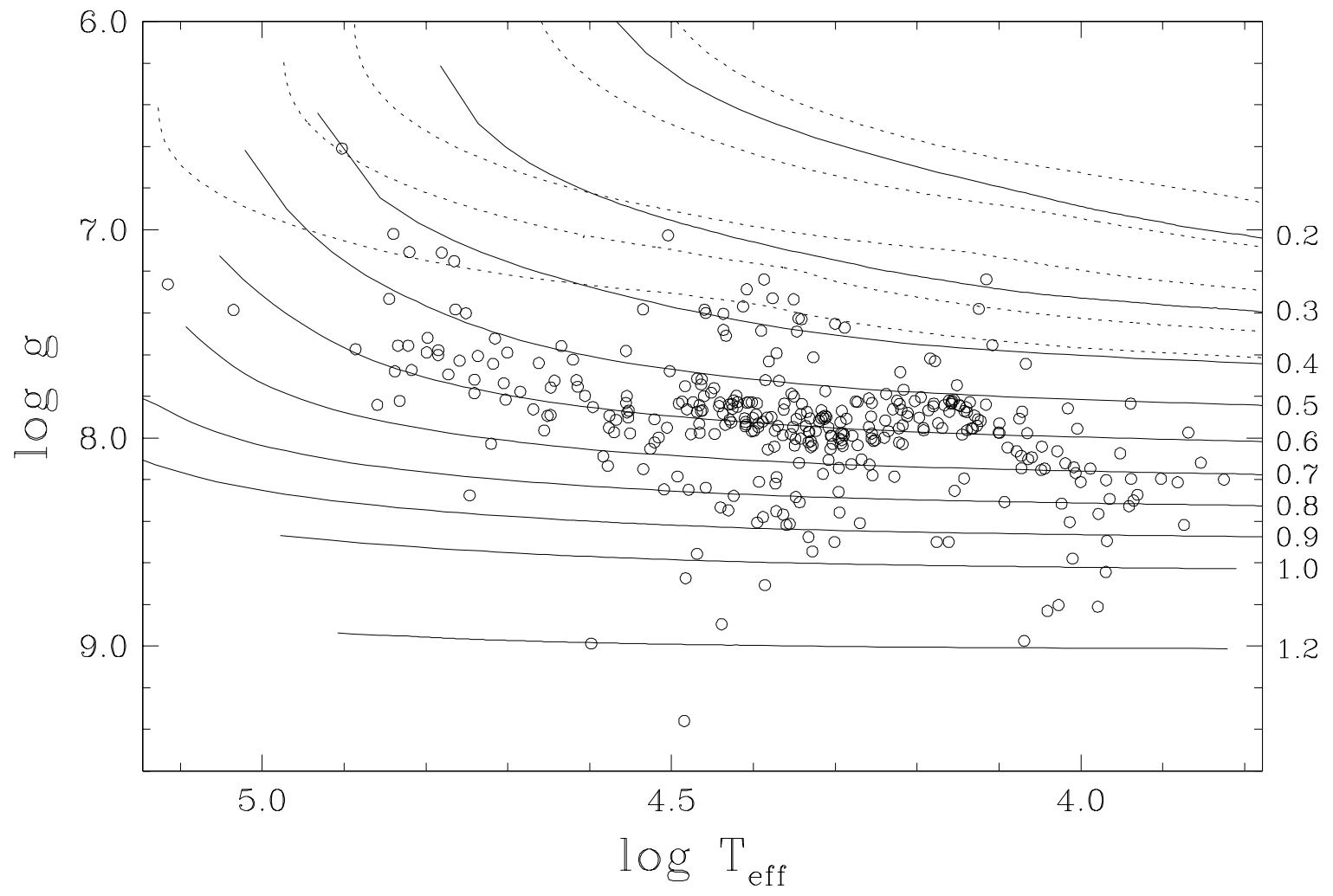

Figure 6 


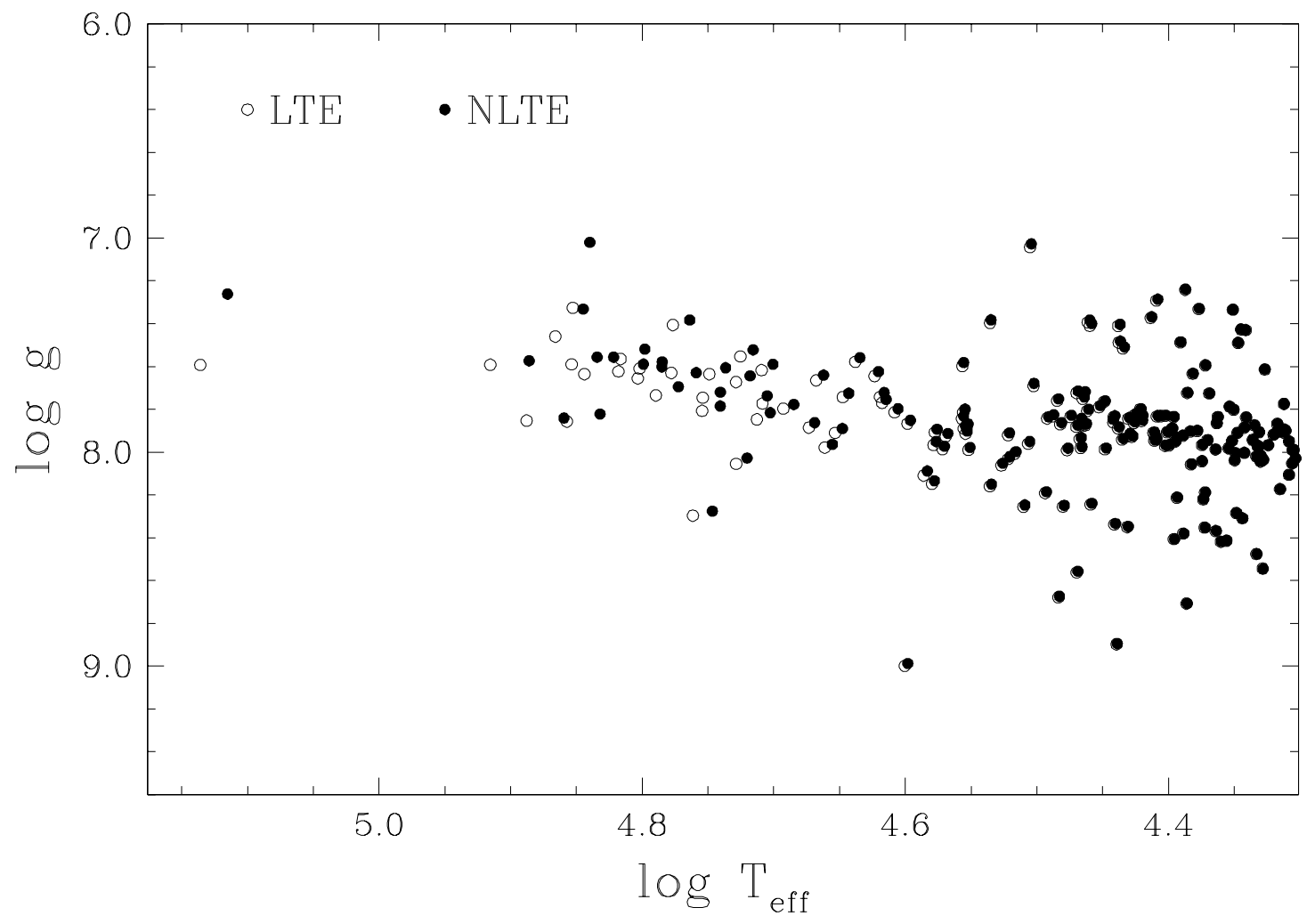

Figure 7 

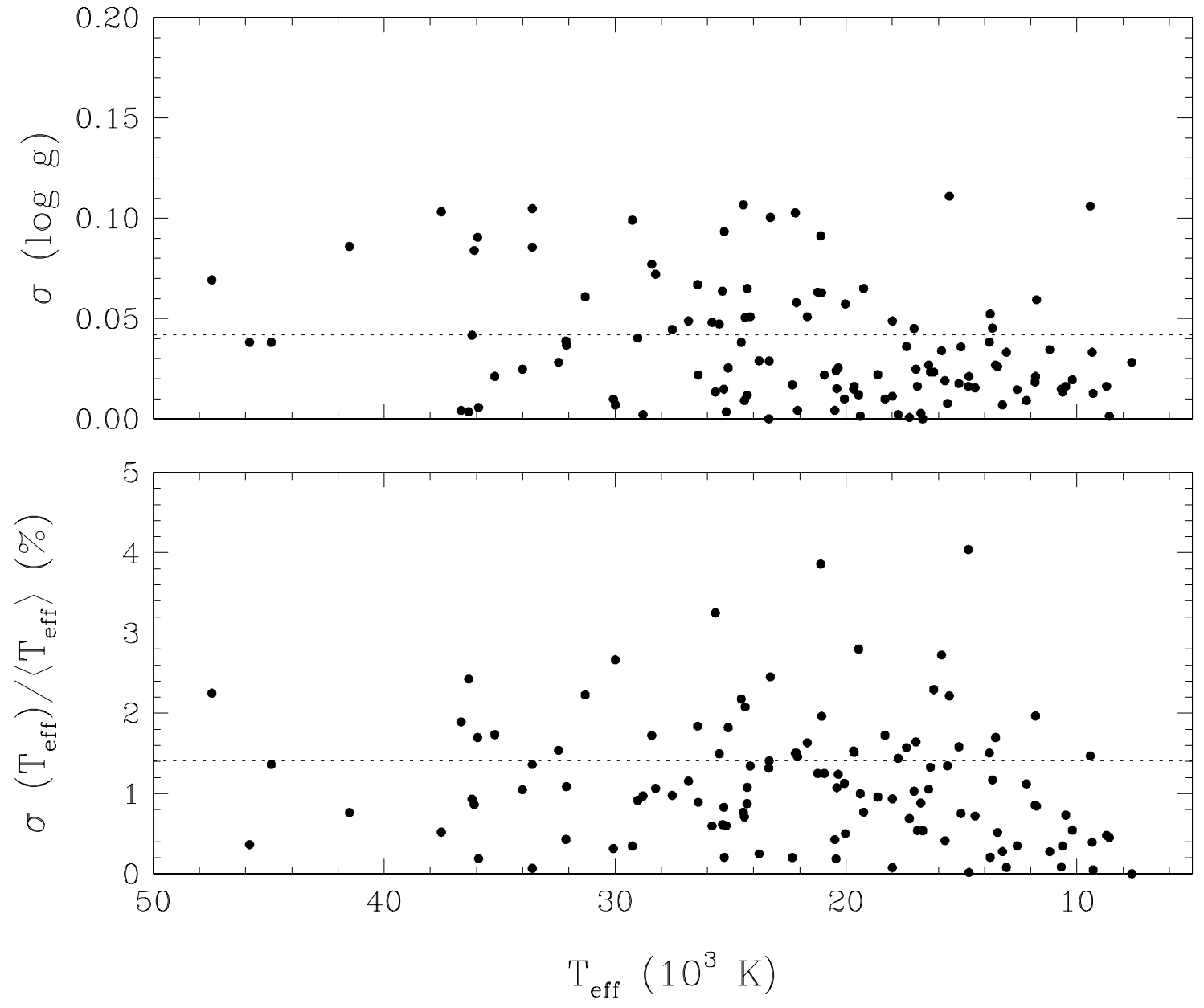

Figure 8 

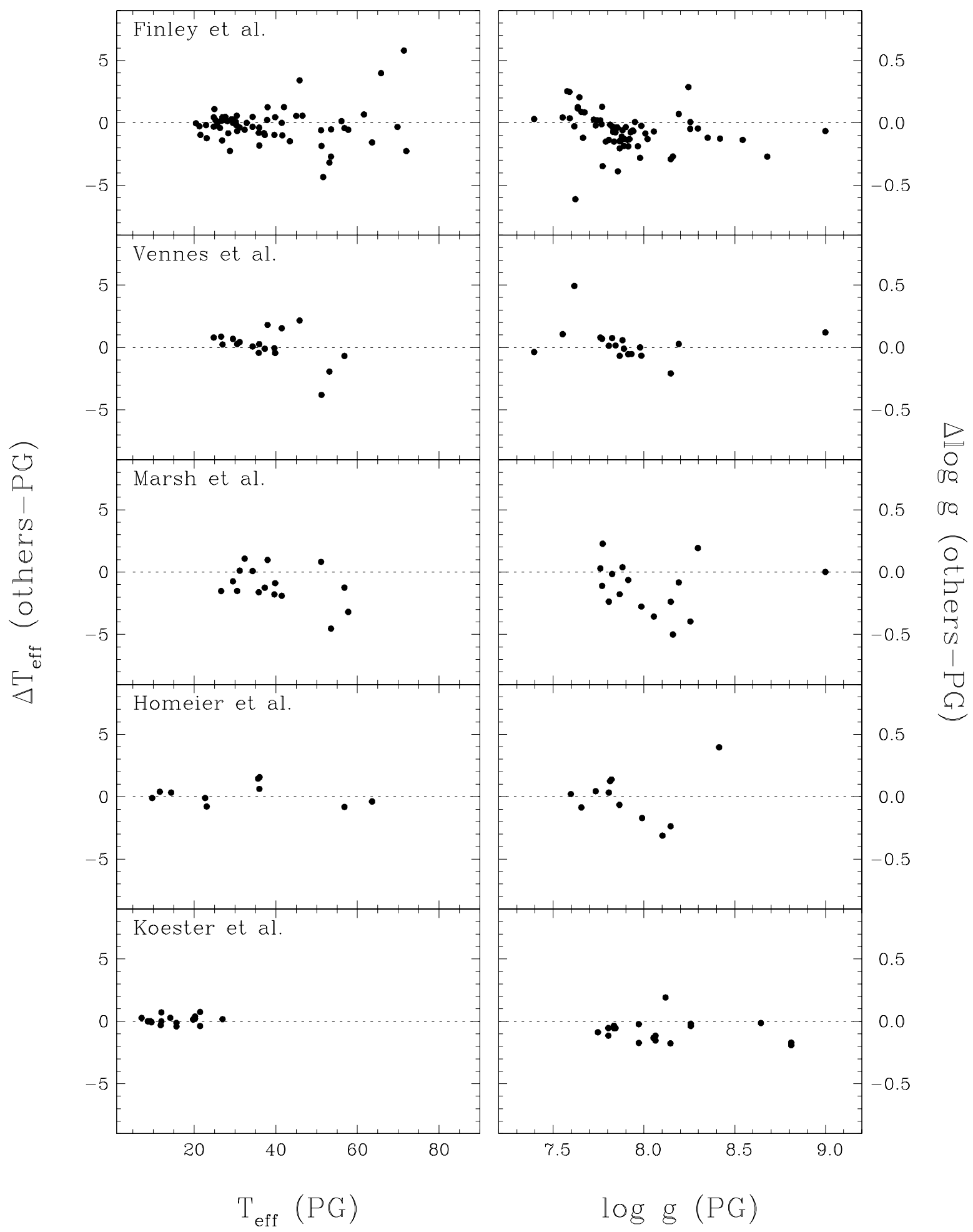

Figure 9 


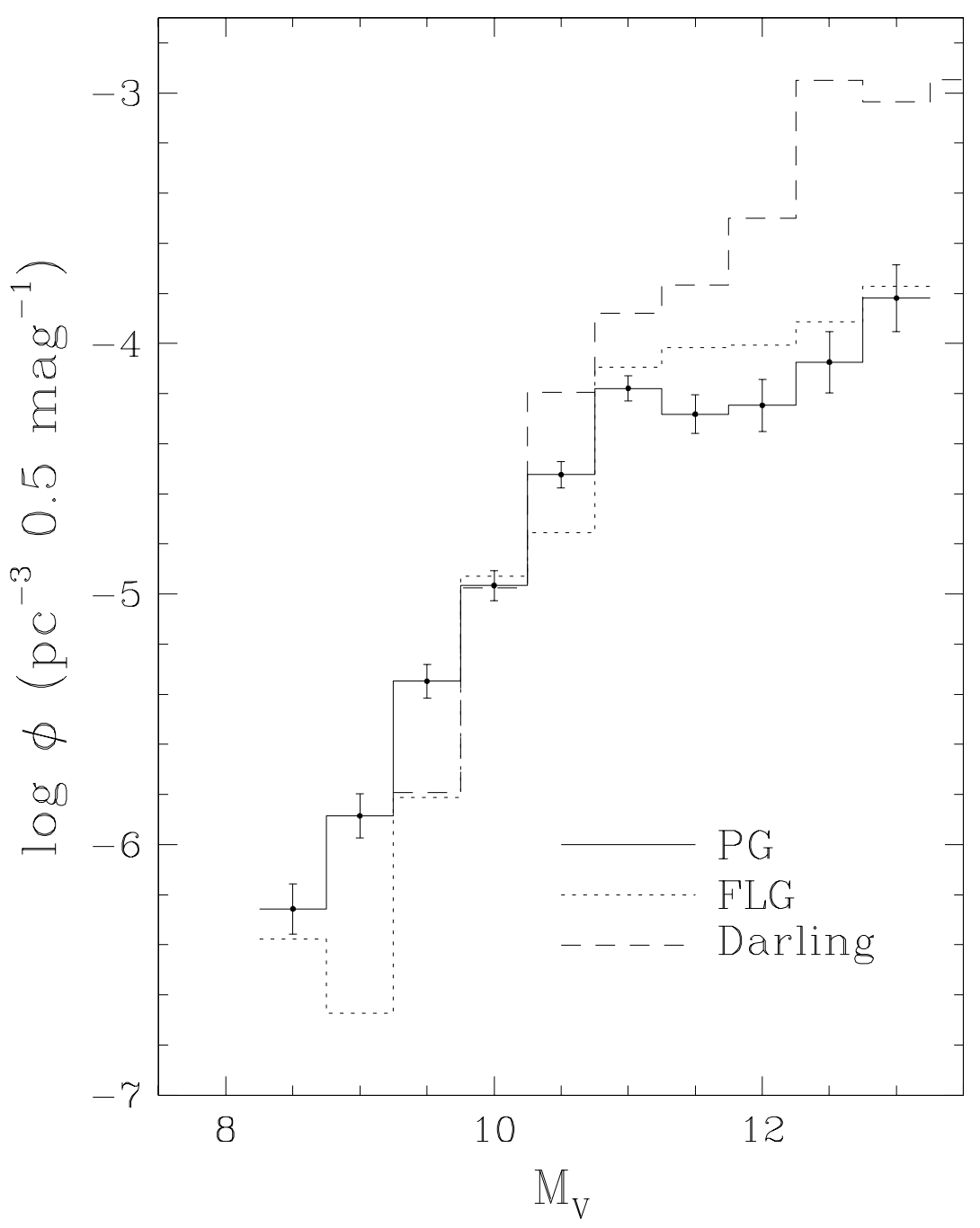

Figure 10 


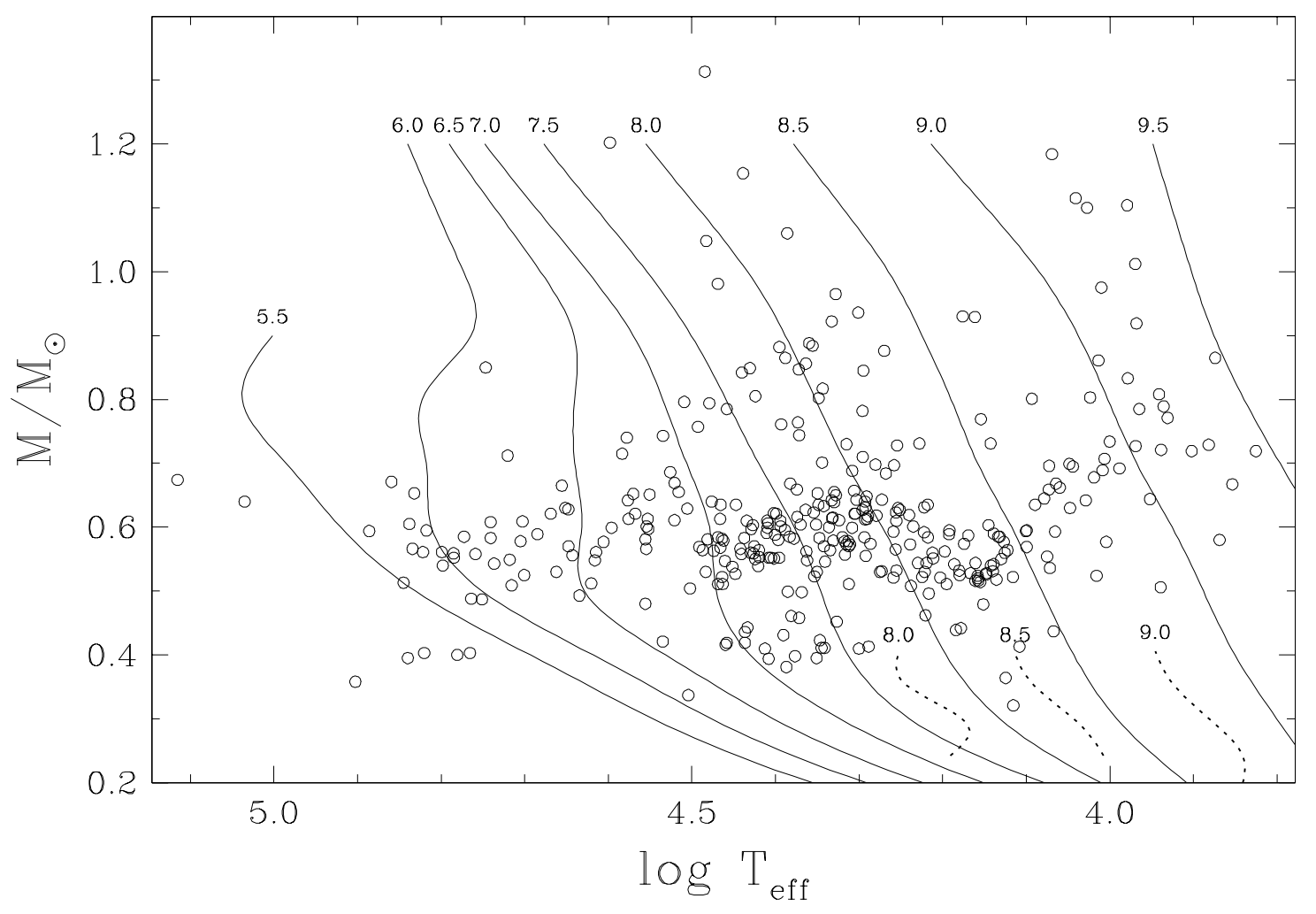

Figure 11 

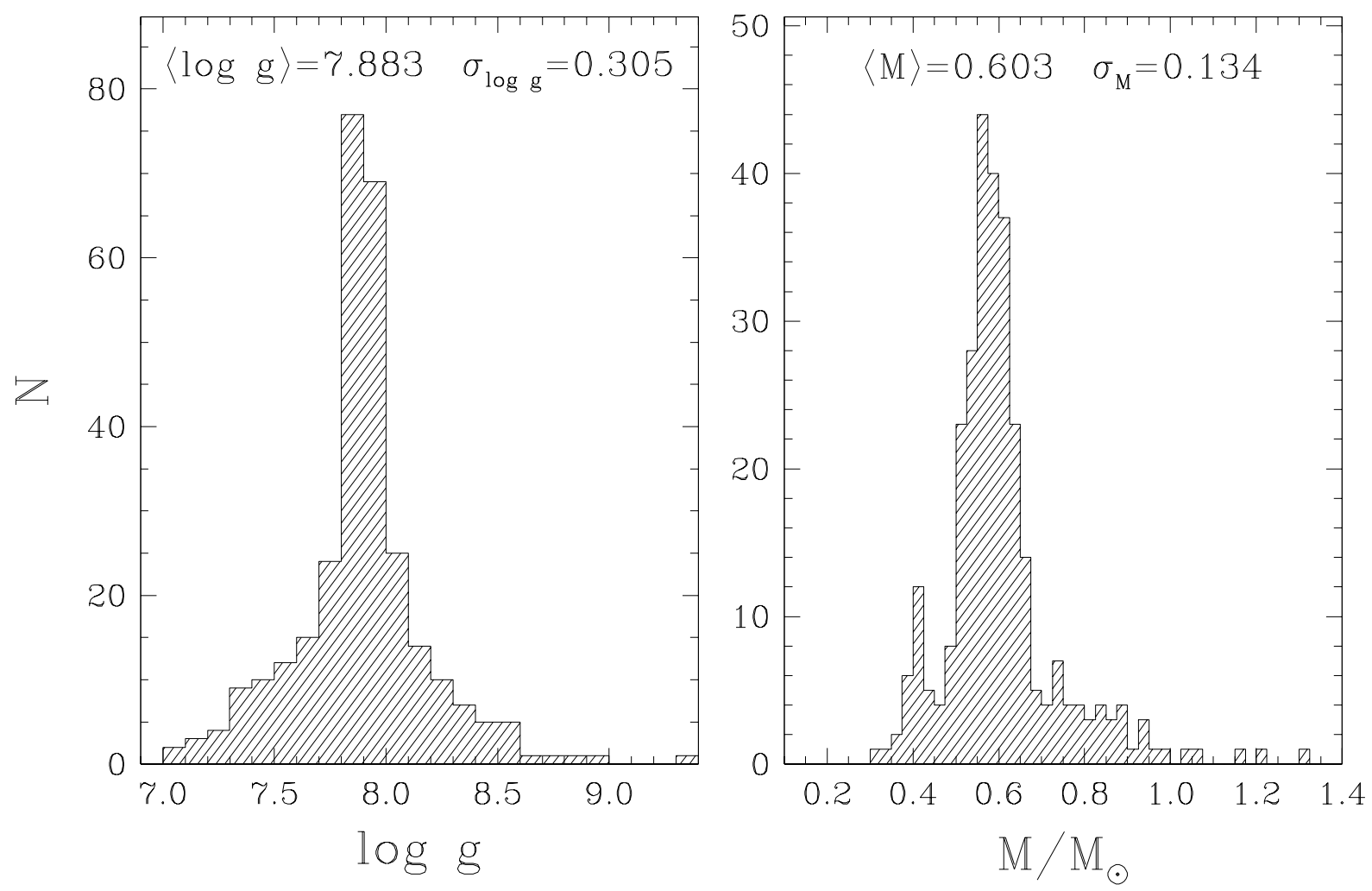

Figure 12 


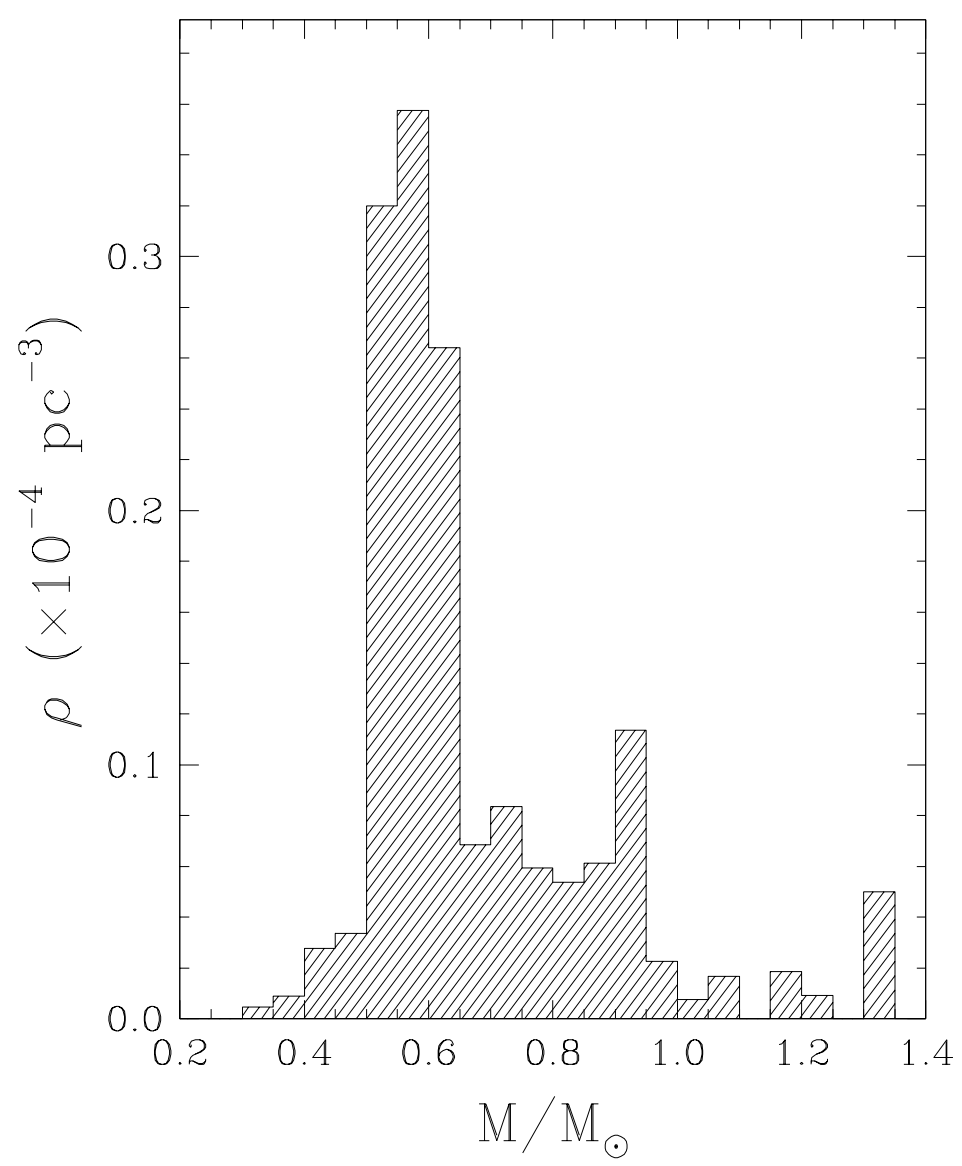

Figure 13 


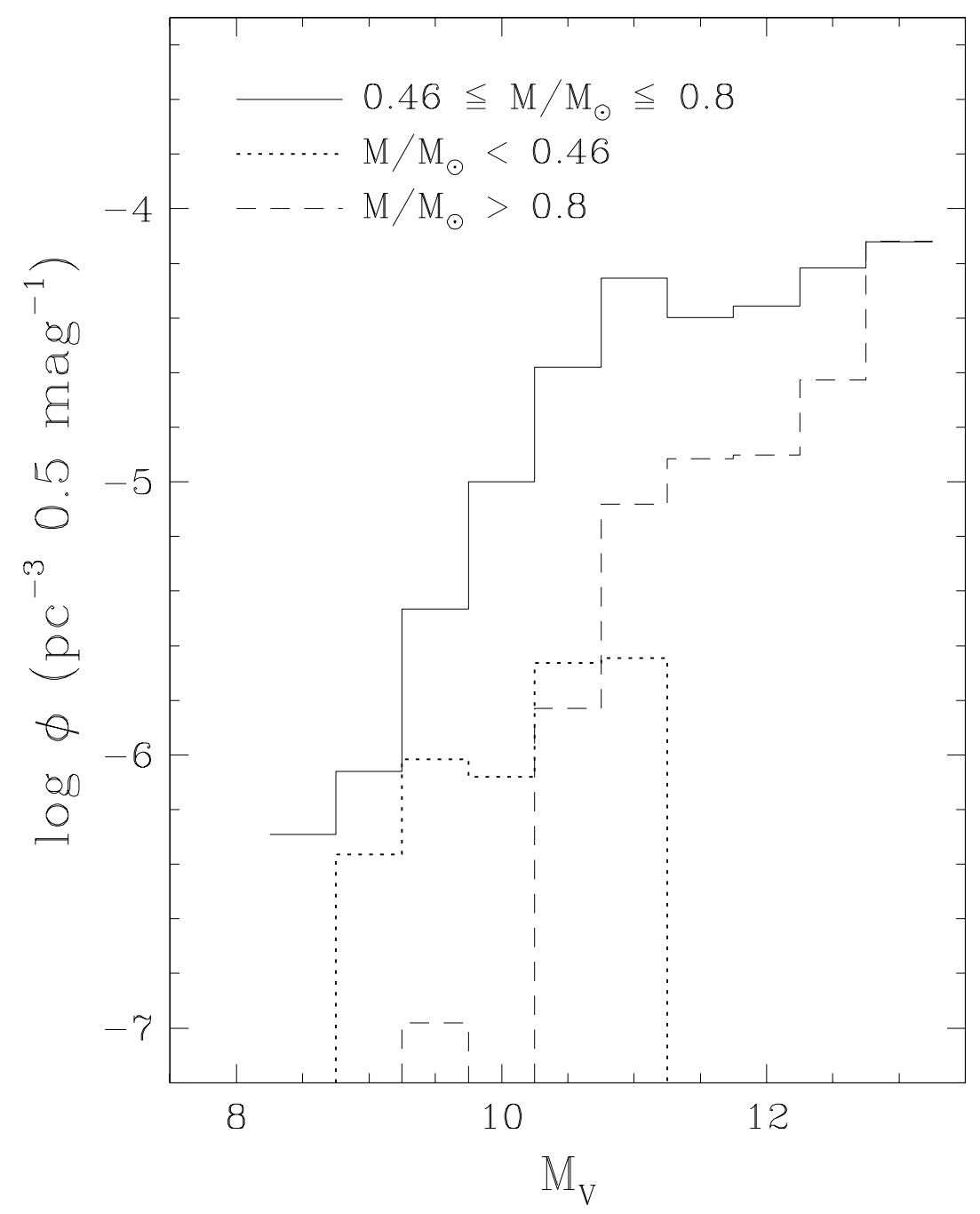

Figure 14 


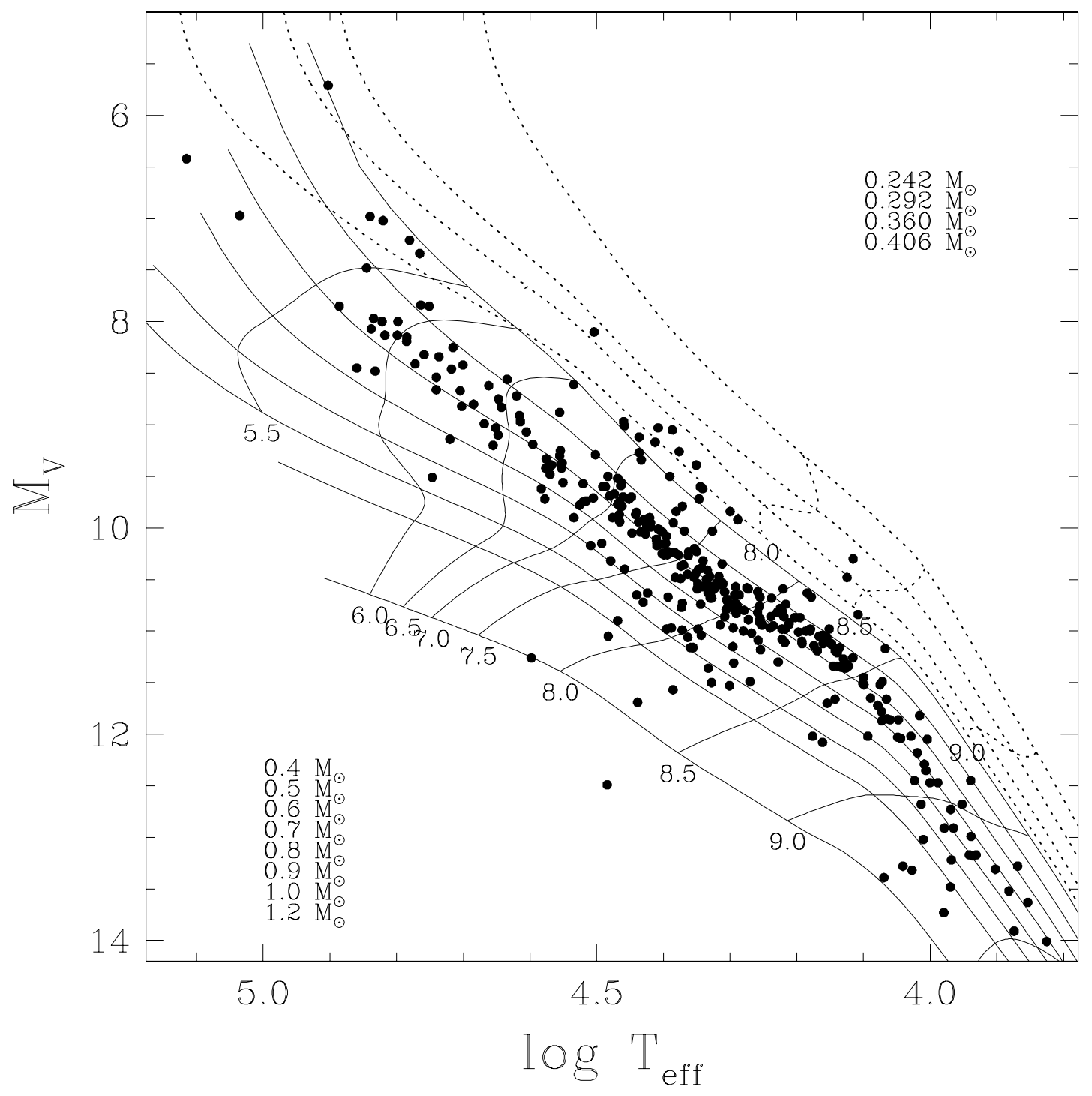

Figure 15 


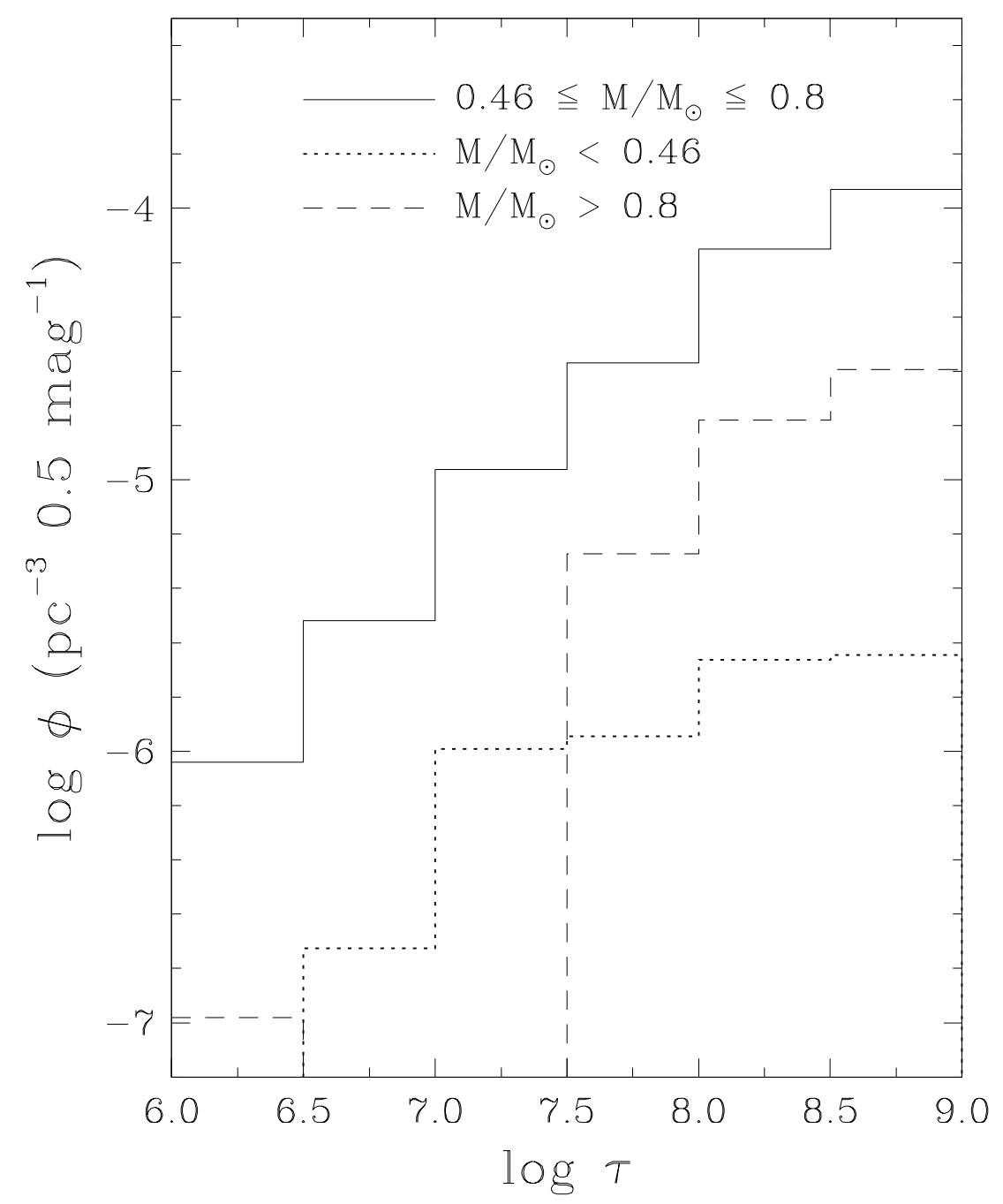

Figure 16 


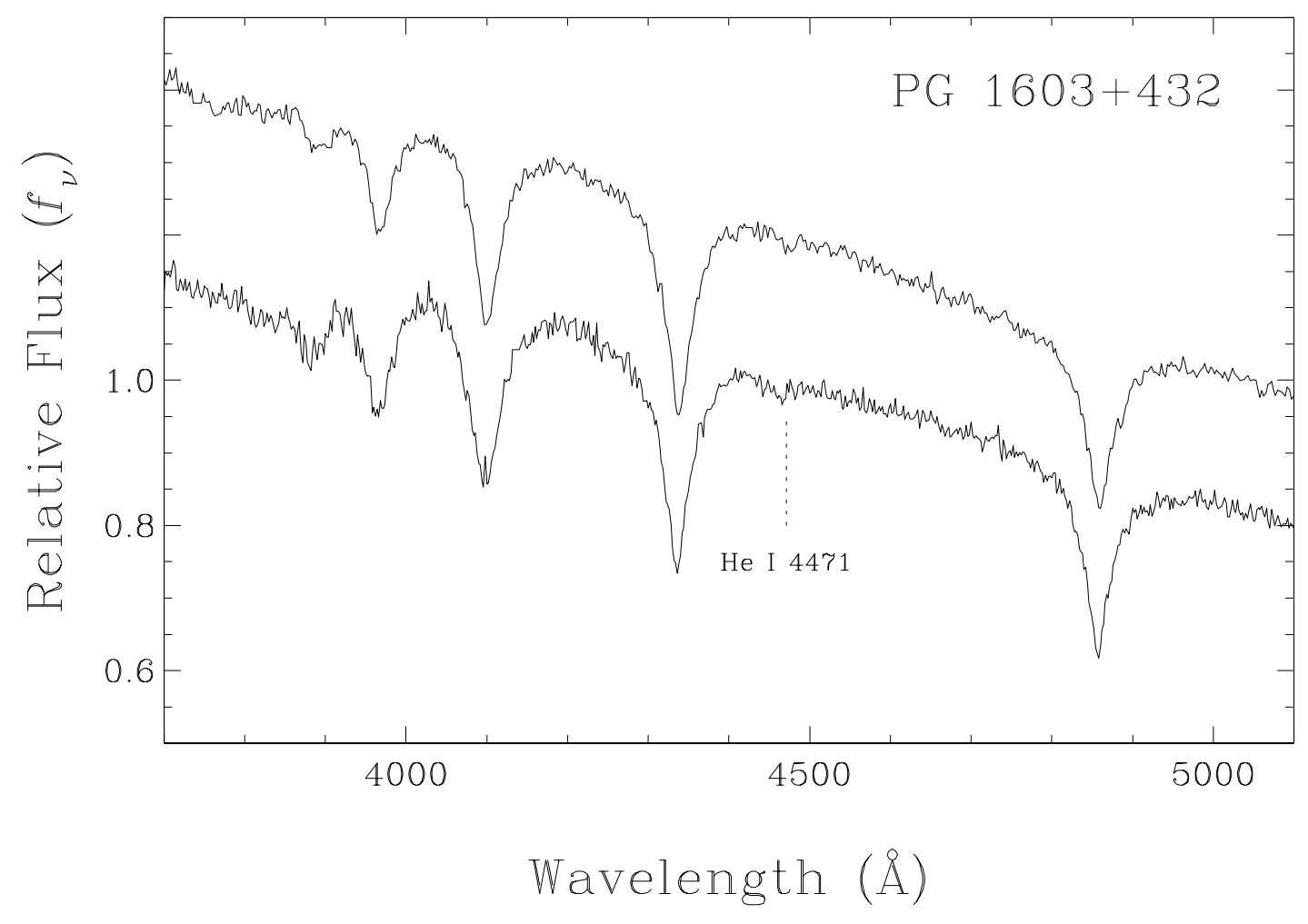

Figure 17 


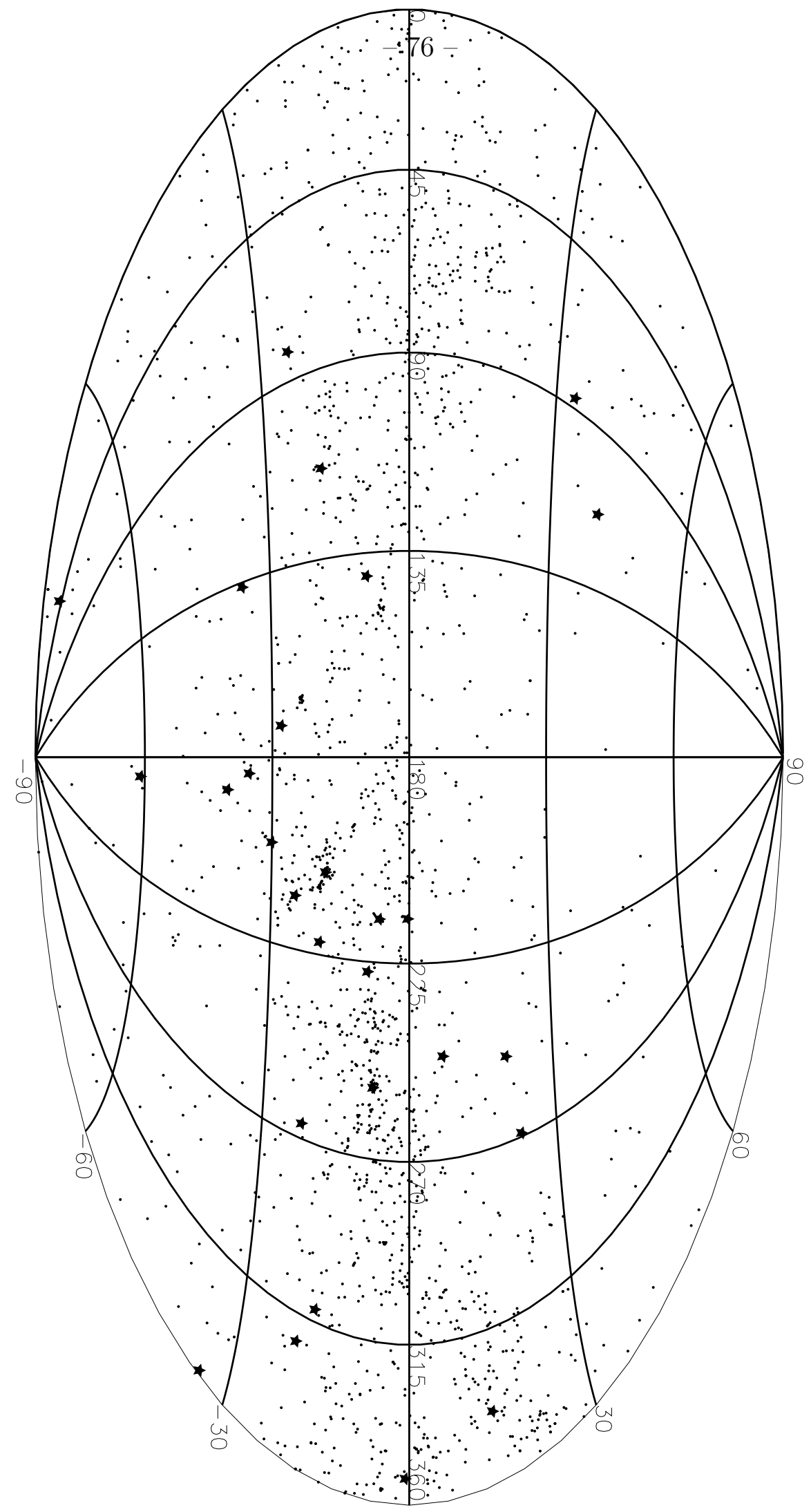

Figure 18 